\title{
MacMahon KZ equation for Ding-lohara-Miki algebra
}

\author{
Panupong Cheewaphutthisakun ${ }^{a}$ and Hiroaki Kanno ${ }^{a, b}$ \\ ${ }^{a}$ Graduate School of Mathematics, Nagoya University, \\ Nagoya, 464-8602, Japan \\ ${ }^{b}$ KMI, Nagoya University, \\ Nagoya, 464-8602, Japan \\ E-mail: panupong . cheewaphutthisakun@gmail.com, \\ kanno@math.nagoya-u.ac.jp
}

ABSTRACT: We derive a generalized Knizhnik-Zamolodchikov equation for the correlation function of the intertwiners of the vector and the MacMahon representations of DingIohara-Miki algebra. These intertwiners are cousins of the refined topological vertex which is regarded as the intertwining operator of the Fock representation. The shift of the spectral parameter of the intertwiners is generated by the operator which is constructed from the universal $R$ matrix. The solutions to the generalized $\mathrm{KZ}$ equation are factorized into the ratio of two point functions which are identified with generalizations of the Nekrasov factor for supersymmetric quiver gauge theories.

Keywords: Conformal and W Symmetry, Conformal Field Theory, Quantum Groups, Supersymmetric Gauge Theory

ArXiv EPRINT: 2101.01420 


\section{Contents}

1 Introduction $\quad \mathbf{2}$

1.1 Strategy of deriving q-KZ equation 3

1.2 Solutions to the generalized KZ equation 4

1.3 Organization of material 4

1.4 Definitions and useful formulas 5

2 Ding-Iohara-Miki algebra 5

2.1 Definition of DIM algebra 6

2.2 Coproduct 7

2.3 Grading operators

2.4 Universal $R$ matrix 8

3 Representations of DIM algebra $\quad 8$

3.1 Vector representations 9

3.2 Tensor product representation of the vector representation 9

$\begin{array}{lll}3.3 & \text { Fock representation } & 11\end{array}$

$\begin{array}{lll}3.4 & \text { MacMahon representation } & 12\end{array}$

$\begin{array}{ll}3.5 & \text { Horizontal Fock representation } \\ \end{array}$

4 Trivalent intertwiners

$\begin{array}{lll}4.1 & \text { Vector intertwiner } & 15\end{array}$

$\begin{array}{lll}4.2 & \text { Fock intertwiner } & 16\end{array}$

$\begin{array}{lll}4.3 & \text { MacMahon intertwiner } & 16\end{array}$

$\begin{array}{lll}4.4 \text { Dual intertwiners } & 17\end{array}$

5 Generalized $\mathrm{KZ}$ equation for the vector intertwiners 20

$\begin{array}{ll}5.1 \text { Construction of the shift operator } 20 & 20\end{array}$

5.2 Commutation of vector intertwiner and the shift operators 22

$5.3 R$-matrix of the vector representation $\quad 25$

5.4 KZ equation for the vector intertwiners 26

5.5 Shift operator from the universal $R$-matrix 28

6 Generalized KZ equation for MacMahon intertwiners 28

6.1 MacMahon shift operator 28

$\begin{array}{lll}6.2 & \text { Effect of the shift operator } & 30\end{array}$

6.3 Case of the dual intertwiner 33

6.4 MacMahon KZ equation 35

7 Solutions to the generalized $\mathrm{KZ}$ equation $\quad 37$

$\begin{array}{lll}7.1 & \text { Correlation function of the vector intertwiners } & 37\end{array}$

$\begin{array}{lll}\text { 7.2 Correlation function of the MacMahon intertwiners } & 38\end{array}$ 


\section{Introduction}

Quantum Knizhnik-Zamolodchikov ( $\mathrm{q}-\mathrm{KZ}$ ) equation is originally introduced for the correlation function of the intertwiners of the quantum affine algebra [19, 26, 30, 41]. In [9] we have shown that one can develop a parallel story for Ding-Iohara-Miki (DIM) algebra [18, 31], which is regarded as the quantum toroidal algebra of $\mathfrak{g l}_{1}$. The trivalent intertwining operator of the Fock representations of DIM algebra agrees with what is called the refined topological vertex $[2,6,7,28]$. Consequently, the correlation function of the intertwiners gives a building block of the Nekrasov partition functions of the five dimensional supersymmetric quiver gauge theories in accord with the AGT correspondence.

Our main interest in the generalized q-KZ equation comes from the AGT correspondence and its generalizations [5, 14, 32, 43], which tell us that the conformal blocks of two dimensional conformal field theories and the instanton partition functions, and hence the low-energy effective actions of the supersymmetric gauge theories are related. This correspondence allows us to study problems on one side from the perspective of the other side. For example, the modular properties of the six dimensional Seiberg-Witten theory with adjoint hypermultiplet can be explained by using the elliptic Knizhnik-Zamolodchikov equation of DIM algebra [10].

In the paper [9], the authors gave a general method of deriving the generalized KZ equation for the Fock intertwiners, and wrote out explicit solutions to the equation. The general solutions are written in terms of a product of the propagators of the Fock intertwiners (the refined topological vertex). Moreover, as expected from the AGT correspondence, the solution relates to the Nekrasov function which gives the instanton partition function. We know from the papers $[20,22]$ that the MacMahon modules are, in a sense, a generalization of the Fock modules. More precisely, the MacMahon modules can be constructed as the inductive limit of an inductive system whose objects are Fock modules. Moreover, we are able to express the MacMahon intertwiner in terms of the Fock intertwiners [11]. Thus, it is natural to ask whether we can generalize the story in [9] to derive the generalized KZ equation for the MacMahon representation of DIM algebra. In this paper we will show this is indeed the case.

Let $\Psi_{\lambda}(u)$ be the intertwiner of the Fock representation of DIM algebra, where $\lambda$ is a Young diagram which labels a basis of the Fock representation and $u$ is the spectral parameter. The $(q, t)-\mathrm{KZ}$ equation derived in [9] implies that the correlation function is expressed as a product of two point functions like the Wick theorem for free fields. One can check that the inverse of the two point function agrees with the Nekrasov factor which is a building block of five dimensional quiver gauge theories with the equivariant parameters $\left(q_{1}, q_{2}\right)=\left(e^{\epsilon_{1}}, e^{\epsilon_{2}}\right),[34,35,39,48]$. Namely, if we define

$$
N_{\lambda \mu}(u, v)=\left\langle\Psi_{\lambda}(u) \Psi_{\mu}(v)\right\rangle^{-1}
$$


then we can show that

$$
\begin{aligned}
& N_{\lambda \mu}(u, v)=\prod_{\substack{\square \in \lambda \\
\square \in \mu}} \frac{\left(1-q_{1} \frac{\chi_{\square}(u)}{\chi \square}(v)\right.}{\left(1-\frac{\chi_{\square}(u)}{\chi_{\square}(v)}\right)\left(1-q_{1} q_{2} \frac{\chi_{\square}(u)}{\chi_{\square}(v)}\right)} \cdot \prod_{\square \in \lambda}\left(1-q_{1} q_{2} \frac{\chi_{\square}(u)}{v}\right) \cdot \prod_{\square \in \mu}\left(1-\frac{u}{\chi_{\square}(v)}\right) \\
& =\prod_{\square \in \lambda}\left(1-q_{1}^{-\ell_{\mu}(\square)} q_{2}^{a_{\lambda}(\square)+1} \frac{u}{v}\right) \prod_{\square \in \mu}\left(1-q_{1}^{\ell_{\lambda}(\mathbf{\square})+1} q_{2}^{-a_{\mu}(\mathbf{\square})} \frac{u}{v}\right),
\end{aligned}
$$

where for $\square=(i, j) \in \lambda$

$$
\chi_{\square}(u):=u \cdot q_{1}^{i-1} q_{2}^{j-1},
$$

and

$$
a_{\mu}(\square)=\mu_{i}-j, \quad \ell_{\mu}(\square)=\mu_{j}^{\vee}-i .
$$

\subsection{Strategy of deriving $\mathrm{q}-\mathrm{KZ}$ equation}

Let $\Psi_{\alpha}(z)$ be the component of the DIM intertwiner in general. Namely $\alpha$ stands for $1 \mathrm{~d}, 2 \mathrm{~d}$ and 3d Young diagrams for the vector, Fock and MacMahon representations, respectively. Our first task is to construct the shift operator which generates the shift of the spectral parameter;

$$
p^{z \partial_{z}} \Psi_{\alpha}(z)=\mathcal{T}_{\alpha}^{-}\left(\mathfrak{q}^{-3 / 2} z\right) \cdot \Psi_{\alpha}(z) \cdot \mathcal{T}_{\alpha}^{+}\left(\mathfrak{q}^{-1 / 2} z\right) .
$$

This is a quantum version of the Sugawara construction $L_{-1} \sim \sum_{n}: J_{n} J_{-n-1}$ : for the classical KZ equation. One of the technical problems in deriving the generalized KZ equation is the construction of such a shift operator. In [9] and [10], the shift operator is identified with the composition of the intertwiner and the dual intertwiner by tuning their spectral parameters appropriately. Remarkably the shift parameter is fixed to be $p=\mathfrak{q}^{-2}=q_{1} q_{2}$. It turns out that up to the normalization, the same shift operator is obtained in terms of the universal $R$ matrix $\mathcal{R}_{0}$ of DIM algebra (see section 2.4);

$$
\mathcal{T}^{+}=\left[\rho^{V} \otimes \rho^{H}\right]\left(\mathcal{R}_{0}\right), \quad \mathcal{T}^{-}=\left[\rho^{H} \otimes \rho^{V}\right]\left(\mathcal{R}_{0}\right),
$$

where $\rho^{V}$ and $\rho^{H}$ are the vertical and the horizontal representations, respectively (see section 3$)$.

The second ingredient is the commutation relations of the intertwiners and the shift operator;

$$
\mathcal{T}_{\alpha}^{ \pm}(z) \Psi_{\beta}(w)=\mathcal{R}_{\beta \alpha}\left(\mathfrak{q}^{n} \frac{z}{w}\right) \Psi_{\beta}(w) \mathcal{T}_{\alpha}^{ \pm}(z),
$$

where $\mathcal{R}_{\beta \alpha}$ is the (diagonal) $R$ matrix of DIM algebra and the power $n$ of $\mathfrak{q}$ depends on $\mathcal{T}_{\alpha}^{ \pm}$. We also have a similar commutation relation for the dual intertwiner $\Psi_{\beta}^{*}(w)$, where we should change the power $n$ appropriately. The relation (1.7) replaces the commutation (OPE) relations of the vertex operators with the current algebra in the classical case. The $R$ matrix appearing in (1.7) can be identified with

$$
\mathcal{R}=\left[\rho^{V_{1}} \otimes \rho^{V_{2}}\right]\left(\mathcal{R}_{0}\right),
$$

which implies that $\mathcal{R}_{\beta \alpha}$ appears in the commutation relation of the intertwiners [8,11]. If we define the shift operator by the composition of $\Psi$ and $\Psi^{*},(1.7)$ follows from this property. 
On the other hand, the commutation relation (1.7) also comes from the fact that both the shift operator and the $R$ matrix are derived from the same object; the universal $R$ matrix.

After all these are prepared, the shift of the spectral parameter of the intertwiners is achieved by an insertion of a pair of the shift operators $\mathcal{T}_{\alpha}^{ \pm}(z)$. Then we can use the commutation relations to move them to the left or the right most position of the strip of the intertwiners, where they act on the vacuum state. In this precess a product of $R$ matrices is produced. Since the shift operators $\mathcal{T}_{\alpha}^{ \pm}(z)$ have only the positive or the negative modes of free bosons, the vacuum is their eigenstate and consequently we obtain a difference equation for the correlation function of the intertwiners, which we identify with a generalized KZ equation for DIM algebra.

\subsection{Solutions to the generalized $\mathrm{KZ}$ equation}

For simplicity, let us assume the correlation function does not involve the dual intetwiners. ${ }^{1}$ In this case our generalized KZ equation for DIM algebra canonically takes the following form

$$
\left\langle\Psi_{\alpha_{1}}\left(z_{1}\right) \cdots \Psi_{\alpha_{k}}\left(p z_{k}\right) \cdots \Psi_{\alpha_{n}}\left(z_{n}\right)\right\rangle=A_{k} \cdot\left\langle\Psi_{\alpha_{1}}\left(z_{1}\right) \cdots \Psi_{\alpha_{k}}\left(z_{k}\right) \cdots \Psi_{\alpha_{n}}\left(z_{n}\right)\right\rangle,
$$

where

$$
A_{k}=\prod_{i<k} R_{\alpha_{i} \alpha_{k}}\left(p^{-1} z_{i} / z_{k}\right)^{-1} \cdot \prod_{k<j} R_{\alpha_{k} \alpha_{j}}\left(z_{k} / z_{j}\right) .
$$

As mentioned before the shift parameter is $p=\mathfrak{q}^{-2}=q_{1} q_{2}$, which is fixed by the choice of the (horizontal) Fock space. This is schematically the same form as the q-KZ equation for the quantum affine algebra $[19,26,30,41]$. Note that, contrary to the case of the quantum affine algebra, the $R$ matrices appearing in eq. (1.9) is diagonal with respect to the label $\alpha$ of basis of the vertical (or evaluation) representation and their ordering does not matter. Due to such an abelian nature of the $R$ matrix, the solutions to our KZ equation are factorized into the ratio of fundamental building blocks which are given by two point functions. We find the two point functions of MacMahon $\mathrm{KZ}$ equation can be regarded as a generalization of the Nakrasov factor (1.2).

\subsection{Organization of material}

The paper is organized as follows; sections $2-4$ are preliminaries. We start by reviewing the definition of DIM algebra and some of its properties in section 2. We introduce the universal $R$ matrix of DIM algebra. Then, in section 3 , we concern with the representations of DIM algebra. The coproduct of DIM algebra is crucial for defining the representation by the tensor product. We show that we can construct the vertical Fock representation from the vector representations which are the simplest vertical representation of DIM algebra. By applying a similar method, we can construct the so-called MacMahon representation from the Fock representation. We also introduce the horizontal Fock representation by using the deformed Heisenberg algebra. In section 4 we give explicit expressions of the

\footnotetext{
${ }^{1}$ For the case with the dual intertwiners, see the main text for detail.
} 
trivalent intertwiners, where vertical representations are vector, Fock, and MacMahon representations, respectively. We also discuss the dual intertwiners.

Before embarking on the task of derivation of the MacMahon $\mathrm{KZ}$ equation, we discuss the vector $\mathrm{KZ}$ equation in section 5, since basic ideas are well-illustrated in a simplified setting. Following the method of the paper [9], we construct the shift operator $\mathcal{T}$ which plays an important role in the derivation. We also show an alternative way to construct the shift operator $\mathcal{T}$ from the universal $R$-matrix, which is useful in our construction of the shift operator in MacMahon case.

Sections 6 and 7 are the main part of the paper. In section 6 we use the method discussed in section 5 to construct the shift operator and provide a derivation of the MacMahon KZ equation. Finally, in section 7, we solve the MacMahon KZ equation and show that the solution can be regarded as a generalized Nekrasov function. Some of the techinical computation are presented in appendix.

\subsection{Definitions and useful formulas}

Before ending the introduction, let us introduce the theta function and also a well-known lemma.

The theta function $\theta_{p}(z)$ is defined by

$$
\theta_{p}(z):=(p ; p)_{\infty}(z ; p)_{\infty}\left(p z^{-1} ; p\right)_{\infty}=(1-z) \prod_{k=1}^{\infty}\left(1-p^{k}\right)\left(1-p^{k} z\right)\left(1-p^{k} z^{-1}\right)
$$

where we use the infinite product;

$$
(x ; p)_{\infty}:=\prod_{j \geq 0}\left(1-p^{j} x\right)=\exp \left(-\sum_{k=1}^{\infty} \frac{x^{k}}{k\left(1-p^{k}\right)}\right) .
$$

It is easy to check

$$
\theta_{p}\left(p^{n} z\right)=(-z)^{n} p^{-\frac{1}{2} n(n-1)} \theta_{p}(z)
$$

Finally, we state a particular case of the Campbell-Baker-Hausdorff (CBH) formula.

Lemma 1.1. If $[A, B]$ is central, i.e. $[[A, B], A]=[[A, B], B]=0$, then

$$
e^{A} e^{B}=e^{B} e^{A} e^{[A, B]} .
$$

This formula is repeatedly used for computing the commutation relations of intertwining operators or the vertex operators.

\section{Ding-Iohara-Miki algebra}

In this section we provide a quick review of the definition of Ding-Iohara-Miki (DIM) algebra, which is the quantum toroidal algebra of $\mathfrak{g l}_{1}$. The material in this and the next section is based on the papers $[11,20]$. 


\subsection{Definition of DIM algebra}

The intriguing triality of DIM algebra becomes manifest by using the parameters $\left(q_{1}, q_{2}, q_{3}\right)$ with $q_{1} q_{2} q_{3}=1$. We assume they are generic in the sense that for any $a, b, c \in \mathbb{Z}$,

$$
q_{1}^{a} q_{2}^{b} q_{3}^{c}=1 \Longrightarrow a=b=c .
$$

We may parametrize $\left(q_{1}, q_{2}, q_{3}\right)$ by $\mathfrak{q}, \mathfrak{d} \in \mathbb{C}$ as follows;

$$
q_{1}=\frac{\mathfrak{d}}{\mathfrak{q}}, \quad q_{2}=\frac{1}{\mathfrak{d} \mathfrak{q}}, \quad q_{3}=\mathfrak{q}^{2} .
$$

But note that this parametrization breaks the triality of DIM algebra. We define the DIM algebra $U_{\mathfrak{q}, \mathfrak{d}}\left(\widehat{\widehat{\mathfrak{g l}}}_{1}\right)$ to be the associative algebra with the generators $E_{k}, F_{k}, K_{0}^{ \pm}, H_{r}(k \in$ $\mathbb{Z}, r \in \mathbb{Z} \backslash\{0\}$ ) and $C$. Introducing the generating functions (currents); ${ }^{2}$

$$
E(z)=\sum_{k \in \mathbb{Z}} E_{k} z^{-k}, \quad F(z)=\sum_{k \in \mathbb{Z}} F_{k} z^{-k}, \quad K^{ \pm}(z)=K_{0}^{ \pm} \exp \left( \pm \sum_{r=1}^{\infty} H_{ \pm r} z^{\mp r}\right),
$$

and the structure function

$$
g(z, w):=\left(z-q_{1} w\right)\left(z-q_{2} w\right)\left(z-q_{3} w\right)
$$

we can write the following defining relations:

$$
\begin{gathered}
C \text { is central, } \\
K_{0}^{+} K_{0}^{-}=1=K_{0}^{-} K_{0}^{+}, \\
K^{ \pm}(z) K^{ \pm}(w)=K^{ \pm}(w) K^{ \pm}(z), \\
\frac{g\left(C^{-1} z, w\right)}{g(C z, w)} K^{-}(z) K^{+}(w)=\frac{g\left(w, C^{-1} z\right)}{g(w, C z)} K^{+}(w) K^{-}(z), \\
g(z, w) K^{ \pm}\left(C^{(1 \mp 1) / 2} z\right) E(w)+g(w, z) E(w) K^{ \pm}\left(C^{(1 \mp 1) / 2} z\right)=0, \\
g(w, z) K^{ \pm}\left(C^{(1 \pm 1) / 2} z\right) F(w)+g(z, w) F(w) K^{ \pm}\left(C^{(1 \pm 1) / 2} z\right)=0, \\
{[E(z), F(w)]=\tilde{g}\left(\delta\left(C \frac{w}{z}\right) K^{+}(z)-\delta\left(C \frac{z}{w}\right) K^{-}(w)\right),} \\
g(z, w) E(z) E(w)+g(w, z) E(w) E(z)=0, \\
g(w, z) F(z) F(w)+g(z, w) F(w) F(z)=0,
\end{gathered}
$$

where the multiplicative delta function is defined by

$$
\delta(z)=\sum_{n \in \mathbb{Z}} z^{n} .
$$

It is convenient to employ the notation

$$
\kappa_{n}:=\prod_{i=1}^{3}\left(q_{i}^{\frac{n}{2}}-q_{i}^{-\frac{n}{2}}\right)=\prod_{i=1}^{3}\left(q_{i}^{n}-1\right)=\prod_{i=1}^{3}\left(1-q_{i}^{-n}\right)=\sum_{i=1}^{3}\left(q_{i}^{n}-q_{i}^{-n}\right),
$$

which satisfies $\kappa_{-n}=-\kappa_{n}$. We choose the normalization of (2.11) as $\tilde{g}=\kappa_{1}^{-1}$.

\footnotetext{
${ }^{2}$ Compared with [11], the normalization of $H_{ \pm r}$ is changed by the factor $\left(\mathfrak{q}-\mathfrak{q}^{-1}\right)$.
} 
Remark 2.1. One should bear in mind that actually there are the Serre's relations in the defining relations. Since we do not use the Serre's relations in this paper, we do not write it out.

By investigating the defining relations we see that $K_{0}^{ \pm}$is central. ${ }^{3}$ Thus we conclude that the DIM algebra has two-dimensional center $\left(C, K_{0}^{ \pm}\right)$. Note that $K_{0}^{+}$is the inverse of $K_{0}^{-}$. We also see that $(2.8)$ implies

$$
\left[H_{r}, H_{s}\right]=\delta_{r+s, 0} \frac{\kappa_{r}}{r}\left(C^{r}-C^{-r}\right) .
$$

\subsection{Coproduct}

The DIM algebra has a "coproduct" $\Delta: U_{\mathfrak{q}, \mathfrak{d}}\left(\widehat{\widehat{\mathfrak{g l}}}_{1}\right) \rightarrow U_{\mathfrak{q}, \mathfrak{d}}\left(\widehat{\widehat{\mathfrak{g l}}}_{1}\right) \otimes U_{\mathfrak{q}, \mathfrak{d}}\left(\widehat{\hat{\mathfrak{g l}}}_{1}\right)$ defined by

$$
\begin{aligned}
\Delta(E(z)) & =E(z) \otimes 1+K^{-}\left(C_{1} z\right) \otimes E\left(C_{1} z\right), \\
\Delta(F(z)) & =F\left(C_{2} z\right) \otimes K^{+}\left(C_{2} z\right)+1 \otimes F(z), \\
\Delta\left(K^{+}(z)\right) & =K^{+}(z) \otimes K^{+}\left(C_{1}^{-1} z\right), \\
\Delta\left(K^{-}(z)\right) & =K^{-}\left(C_{2}^{-1} z\right) \otimes K^{-}(z), \\
\Delta(C) & =C \otimes C,
\end{aligned}
$$

where $C_{1}=C \otimes 1$ and $C_{2}=1 \otimes C$.

We will see that the coproduct structure plays an important role in defining the tensor product representation of DIM. However, we would like to give a remark here that $\Delta$ is not a coproduct in the strict sense. The reason is that it contains an infinite summation of elements which is not defined in general. Hence, when we use $\Delta$ for defining the action of tensor product representation, we have to check every time that the action is well-defined.

\subsection{Grading operators}

The DIM algebra has a bi-grading defined by two grading operators $d_{1}, d_{2}$ which satisfy

$$
\begin{aligned}
& {\left[d_{1}, E(z)\right]=-E(z), \quad\left[d_{1}, F(z)\right]=F(z), \quad\left[d_{1}, H(z)\right]=0,} \\
& {\left[d_{2}, E(z)\right]=z \partial_{z} E(z), \quad\left[d_{2}, F(z)\right]=z \partial_{z} F(z), \quad\left[d_{2}, H(z)\right]=z \partial_{z} H(z) .}
\end{aligned}
$$

In [23] the degrees with respect to $d_{1}$ and $d_{2}$ are called principal degree and homogeneous degree, respectively. Hence, the degree of the vertical spectral parameter $z$ counts the $d_{2}$-grading and the generators $E_{k}, F_{k}$ and $H_{r}$ have gradings $(-1, k),(1, k)$ and $(0, r)$, respectively. The generators with higher $d_{1}$-grading are given by multiple commutators of $E_{k}$ and $F_{k}$. Later we will introduce the horizontal spectral parameter $u$ which counts the $d_{1^{-}}$ grading. The $\mathrm{SL}(2, \mathbb{Z})$ automorphism of the DIM algebra acts on this bi-grading [31]. The grading operators are important in the expression of the universal $R$-matrix which is the main tool for deriving the generalized Knizhnik-Zamolodchikov equation for MacMahon intertwiner.

\footnotetext{
${ }^{3} \operatorname{In}[23] K_{0}^{-}$is denoted by $C^{\perp}$.
} 


\section{$2.4 \quad$ Universal $R$ matrix}

The quantum toroidal algebra allows a quantum (Drinfeld) double construction [17]. Consequently it has a quasi-triangular structure, which implies the existence of a universal $R$ matrix. According to [23] (see also [36]), the universal $R$-matrix $\mathcal{R}$ of DIM algebra factorizes as follows;

$$
\mathcal{R}=\mathfrak{q}^{c^{\perp} \otimes d^{\perp}+d^{\perp} \otimes c^{\perp}} \mathcal{R}_{+} \mathcal{R}_{0} \mathcal{R}_{-},
$$

where $\mathfrak{q}^{c^{\perp}}=K_{0}^{-}$and $d^{\perp}=d_{1}$ (the grading operator for the principal degree). What is most relevant in the present paper is the Cartan factor of $\mathcal{R} ;{ }^{4}$

$$
\mathfrak{q}^{c^{\perp} \otimes d^{\perp}+d^{\perp} \otimes c^{\perp}} \cdot \mathcal{R}_{0}=\left(K_{0}^{-} \otimes \mathfrak{q}^{d_{1}}\right)\left(\mathfrak{q}^{d_{1}} \otimes K_{0}^{-}\right) \exp \left\{\sum_{n=1}^{\infty} n \kappa_{n} h_{-n} \otimes h_{n}\right\}
$$

where $h_{ \pm n}$ is defined via $\kappa_{n} h_{ \pm n}= \pm H_{ \pm n}$. Note that the universal $R$ matrix we use in the present paper is $P \mathcal{R}$ with $P(a \otimes b)=b \otimes a$ in [23].

It is known that DIM algebra acts on the equivariant $K$-theory; $\oplus_{n=0}^{\infty} K_{G}\left(\operatorname{Hilb}^{n}\left(\mathbb{C}^{2}\right)\right)$ of the Hilbert schemes of $n$ points on $\mathbb{C}^{2}$. Hence, we can also define the $R$-matrix by using the ideas coming from geometry [51]. It is interesting to find that the $R$-matrix $\left[\rho^{V_{1}} \otimes \rho^{V_{2}}\right]\left(\mathcal{R}_{0}\right)$ featured in our generalized $\mathrm{KZ}$ equation (1.9) coincides with the infinite slope $R$-matrix $R_{\infty}$ which is ubiquitous in the Khoroshkin-Tolstoy factorization of the slope $s R$-matrix introduced in [52]. As noticed in [52] $R_{\infty}$ corresponds to multiplication by a class of normal bundles in $K$-theory and is diagonal in the fixed point basis of the torus action. It is an intriguing challenge to work out a possible link of the quantum difference equation (1.9) to those in [52].

\section{Representations of DIM algebra}

In this section we concern with the representation of DIM algebra. Note that in this paper we use the word representation and module interchangeably. We start this section with the definition of level.

Definition 3.1 (Level). Let $V$ be a representation of DIM algebra $U_{\mathfrak{q}, \mathfrak{d}}\left(\widehat{\hat{\mathfrak{g}}}_{1}\right)$. We say that the representation $V$ is of level $\left(\gamma_{1}, \gamma_{2}\right) \in \mathbb{C}^{2}$ if $C$ and $K_{0}^{-}$act as constant multiplications by $\gamma_{1}$ and $\gamma_{2}$, respectively.

We will call a representation with $\gamma_{1}=1$ vertical representation. Note that the condition $\gamma_{1}=1$ is kept intact under taking the tensor product. By (2.16) $H_{r}$ are mutually commuting for the vertical representation. Hence the vertical representation allows a basis which simultaneously diagonalizes the Cartan modes $H_{r}$. There are three natural vertical representations of DIM algebra; vector, Fock and MacMahon representations. A basis which diagonalizes $H_{r}$ is labeled by $1 \mathrm{~d}, 2 \mathrm{~d}$ and $3 \mathrm{~d}$ Young diagrams.

\footnotetext{
${ }^{4}$ The definition of $\kappa_{n}$ in this paper is $-\kappa_{n}$ in [23].
} 


\subsection{Vector representations}

We start with the vector representation which is considered as the simplest vertical representation. Though DIM algebra is completely symmetric in parameters $\left(q_{1}, q_{2}, q_{3}\right)$, the symmetry is broken at the level of representation in general. In order to define the vector representation we have to choose one of three parameters as "prefered". Accordingly there are three kinds of vector representations $\rho^{V^{(k)}}(k=1,2,3)[46]$. For a parameter $v \in \mathbb{C}$ we consider a vector space $V(v)$ over $\mathbb{C}$ with a basis $\left\{[v]_{i} \mid i \in \mathbb{Z}\right\}$. The vector representation $V^{(k)}$ is defined as follows:

$$
\begin{aligned}
K^{+}(z)[v]_{i} & =\tilde{\psi}_{k}\left(q_{k}^{i} v / z\right)[v]_{i}, \\
K^{-}(z)[v]_{i} & =\tilde{\psi}_{k}\left(q_{k}^{-i-1} z / v\right)[v]_{i}, \\
E(z)[v]_{i} & =\left(1-q_{k}\right)^{-1} \delta\left(q_{k}^{i+1} v / z\right)[v]_{i+1}, \\
F(z)[v]_{i+1} & =\left(1-q_{k}^{-1}\right)^{-1} \delta\left(q_{k}^{i+1} v / z\right)[v]_{i} .
\end{aligned}
$$

Here

$$
\tilde{\psi}_{1}(z)=\frac{\left(1-q_{2}^{-1} z\right)\left(1-q_{3}^{-1} z\right)}{(1-z)\left(1-q_{1} z\right)}
$$

and $\tilde{\psi}_{2}(z)$ and $\tilde{\psi}_{3}(z)$ are defined by the cyclic permutation of $\left(q_{1}, q_{2}, q_{3}\right)$. The original vector representation in [20] is $\rho^{V^{(1)}}$. In the following we will choose the same one and simply denote it by $\rho^{V}$. If we introduce $\psi(z)=\mathfrak{q} \frac{1-q_{3}^{-1} z}{1-z}$, then we can express the $\tilde{\psi}_{1}(z) \operatorname{as}^{5}$

$$
\tilde{\psi}_{1}(z)=\psi(z) \psi\left(q_{2}^{-1} z\right)^{-1}
$$

It is $\psi(z)$ rather than $\tilde{\psi}_{1}(z)$ which plays a main role later, for example see eqs. (3.22) and (3.23) in section 3.3. It is straightforward to show that $V(v)$ with the above action really forms an irreducible representation with level $(1,1)$.

Remark 3.2. We give two remarks here.

1. In the equations (3.1)-(3.4), $\tilde{\psi}$ and $\delta$ are formal power series.

2. The vector representation is not a highest-weight representation.

\subsection{Tensor product representation of the vector representation}

Now perform the tensor product

$$
V\left(v_{1}\right) \otimes V\left(v_{2}\right) \otimes \cdots \otimes V\left(v_{n}\right)
$$

\footnotetext{
${ }^{5}$ Note that we can exchange $q_{2}$ and $q_{3}$.
} 
So we obtain a representation of $\underbrace{U_{\mathfrak{q}, \mathfrak{d}}\left(\widehat{\widehat{\mathfrak{g l}}}_{1}\right) \otimes \cdots \otimes U_{\mathfrak{q}, \mathfrak{o}}\left(\widehat{\hat{\mathfrak{g l}}}_{1}\right)}_{n \text { times }}$. By using $\Delta^{n-1}$,

$$
\begin{aligned}
\Delta^{n-1}\left(K^{ \pm}(z)\right) & =\overbrace{K^{ \pm}(z) \otimes \cdots \otimes K^{ \pm}(z)}^{n}, \\
\Delta^{n-1}(E(z)) & =\sum_{k=1}^{n} \overbrace{K^{-}(z) \otimes \cdots K^{-}(z)}^{k-1} \otimes E(z) \otimes \overbrace{1 \otimes \cdots \otimes 1}^{n-k}, \\
\Delta^{n-1}(F(z)) & =\sum_{k=1}^{n} \overbrace{1 \otimes \cdots \otimes 1}^{k-1} \otimes F(z) \otimes \overbrace{K^{+}(z) \otimes \cdots \otimes K^{+}(z)}^{n-k} .
\end{aligned}
$$

we obtain a representation of $U_{\mathfrak{q}, \mathfrak{d}}\left(\widehat{\widehat{\mathfrak{g l}}}_{1}\right)$.

However, there are two issues here. First, we see from eq. (3.9) that the action of $E(z)$ results in a product of the formal power series $\tilde{\psi}$ and $\delta$ which is not defined in general, since it contains an infinite summation of elements in we obtain a representation of $U_{\mathfrak{q}, \mathfrak{d}}\left(\widehat{\mathfrak{g}}_{1}\right)$. The solution to this issue is to perform a regularization. More precisely, in the tensor product representation we treat $\tilde{\psi}$ to be a function instead of formal power series.

Second, after regularization, $\tilde{\psi}$ is a function. From eq. (3.5), we see that it contains poles. If we choose $v_{1}, v_{2}, \ldots, v_{n}$ not carefully, then it might hit the poles generated from $\tilde{\psi}$. This means that the parameters $v_{1}, v_{2}, \ldots, v_{n}$ can not be chosen arbitrarily. Then, it is natural to ask what is a condition for $v_{1}, v_{2}, \ldots, v_{n}$ to assure that the action defined by $\Delta^{n-1}$ on $V\left(v_{1}\right) \otimes V\left(v_{2}\right) \otimes \cdots \otimes V\left(v_{n}\right)$ does not hit the poles. An answer is given in the following lemma which we take from the paper [20].

Lemma 3.3. If the parameters $v_{1}, v_{2}, \ldots, v_{n} \in \mathbb{C}$ satisfy the condition that for any $1 \leq$ $i<j \leq n$,

$$
\frac{v_{j}}{v_{i}} \neq q_{1}^{k} \quad \forall k \in \mathbb{Z}
$$

then $V\left(v_{1}\right) \otimes V\left(v_{2}\right) \otimes \cdots \otimes V\left(v_{n}\right)$ is a well-defined representation of DIM algebra.

As a consequence of Lemma 3.3, we get that the representation $V^{n}(v)$ defined by

$$
V^{n}(v)=V(v) \otimes V\left(q_{2} v\right) \otimes \cdots \otimes V\left(q_{2}^{n-1} v\right)
$$

is well-defined. For each $\lambda=\left(\lambda_{1}, \ldots, \lambda_{n}\right) \in \mathbb{Z}^{n}$, we define the state

$$
|\lambda\rangle:=[v]_{\lambda_{1}-1} \otimes\left[q_{2} v\right]_{\lambda_{2}-1} \otimes \cdots \otimes\left[q_{2}^{n-1} v\right]_{\lambda_{n}-1} .
$$

It is easy to see that $\left\{|\lambda\rangle \mid \lambda=\left(\lambda_{1}, \ldots, \lambda_{n}\right) \in \mathbb{Z}^{n}\right\}$ forms a basis of $V^{n}(v)$.

Note that the representation $V^{n}(v)$ is reducible since it contains a nonzero proper DIM-submodule $W^{n}(v)$ which is defined by

$$
W^{n}(v)=\operatorname{span}_{\mathbb{C}}\left\{|\lambda\rangle \mid \lambda \in \mathcal{P}^{n}\right\} .
$$

Here

$$
\mathcal{P}^{n}=\left\{\lambda=\left(\lambda_{1}, \cdots, \lambda_{n}\right) \in \mathbb{Z}^{N} \mid \lambda_{1} \geq \cdots \geq \lambda_{n}\right\}
$$




\subsection{Fock representation}

Once we have constructed the $n$-th tensor product representation $V^{n}(v)$ for any $n \in \mathbb{Z}^{\geq 1}$, it is natural to think about the representation of DIM algebra which collects all of the $V^{n}(v)\left(n \in \mathbb{Z}^{\geq 1}\right)$ together under an identification

$$
\left|\left(\lambda_{1}, \ldots, \lambda_{n}\right)\right\rangle \sim\left|\left(\lambda_{1}, \ldots, \lambda_{n}, 0\right)\right\rangle \sim\left|\left(\lambda_{1}, \ldots, \lambda_{n}, 0,0\right)\right\rangle \sim \cdots
$$

and so on. This recalls us the notion of inductive limit. Of course, to perform the inductive limit we need to have the inductive system in our hand first.

Here we construct the inductive system. First we define $W^{n,+}(v)$ to be the subspace

$$
\operatorname{span}_{\mathbb{C}}\left\{|\lambda\rangle \mid \lambda \in \mathcal{P}^{n,+}\right\} \subseteq V^{n}(v)
$$

where $\mathcal{P}^{n,+}=\left\{\lambda=\left(\lambda_{1}, \cdots, \lambda_{n}\right) \in \mathbb{Z}^{N} \mid \lambda_{1} \geq \cdots \geq \lambda_{n} \geq 0\right\}$. Then we construct the inductive system of vector spaces.

$$
W^{1,+}(v) \stackrel{\tau_{1}}{\longrightarrow} W^{2,+}(v) \stackrel{\tau_{2}}{\longrightarrow} W^{3,+}(v) \cdots
$$

where $\tau_{n}: W^{n,+}(v) \rightarrow W^{n+1,+}(v)$ sends $\left|\left(\lambda_{1}, \ldots, \lambda_{n}\right)\right\rangle$ to $\left|\left(\lambda_{1}, \ldots, \lambda_{n}, 0\right)\right\rangle$. Then, we take the inductive limit of the above inductive system.

$$
\mathcal{F}(v)=\lim _{\longrightarrow} W^{n,+}(v) .
$$

Being an inductive limit, the vector space $\mathcal{F}(v)$ is spanned by $\left\{|\lambda\rangle \mid \lambda \in \mathcal{P}^{+}\right\}$where

$$
\mathcal{P}^{+}=\left\{\lambda=\left(\lambda_{1}, \lambda_{2}, \ldots\right) \mid \lambda_{i} \geq \lambda_{i+1}, \lambda_{i} \in \mathbb{Z}, \lambda_{i}=0 \text { for sufficiently large } i\right\} .
$$

The next task is to endow the structure of $U_{\mathfrak{q}, \mathfrak{d}}\left(\widehat{\widehat{\mathfrak{g l}}}_{1}\right)$-module on $\mathcal{F}(v)$. From eq. (3.18), we know that $\mathcal{F}(v)$ is the disjoint union of $W^{n,+}(v)\left(n \in \mathbb{Z}^{\geq 1}\right)$ modulo the identification $\sim$ in (3.15). Accordingly, it is natural to use the $U_{\mathfrak{q}, \mathfrak{o}}\left(\widehat{\hat{\mathfrak{g}}}_{1}\right)$-module structure on each $W^{n,+}$ to construct the $U_{\mathfrak{q}, \mathfrak{d}}\left(\widehat{\widehat{\mathfrak{g l}}}_{1}\right)$-module structure on $\mathcal{F}(v)$. More precisely, if a partition $\lambda=$ $\left(\lambda_{1}, \lambda_{2}, \ldots, \lambda_{l}, 0,0, \ldots\right)$, then we regard $|\lambda\rangle$ as an element of $V^{l}(v)$ and use the $U_{\mathfrak{q}, \mathfrak{d}}\left(\widehat{\hat{\mathfrak{g l}}}_{1}\right)$ module structure of $V^{l}(v)$.

Unfortunately, the above action is not compatible with the inductive system (3.17). That is, if we denote the representation $V^{l}$ by $\rho^{l}: U_{\mathfrak{q}, \mathfrak{d}}\left(\widehat{\hat{\mathfrak{g l}}}_{1}\right) \rightarrow$ End $V^{l}$ and if $\lambda=$ $\left(\lambda_{1}, \lambda_{2}, \ldots, \lambda_{l}, 0,0, \ldots\right)$, then we find that

$$
\rho^{l}\left(K^{ \pm}(z)\right)\left|\left(\lambda_{1}, \lambda_{2}, \ldots, \lambda_{l}\right)\right\rangle \neq \rho^{l+1}\left(K^{ \pm}(z)\right)\left|\left(\lambda_{1}, \lambda_{2}, \ldots, \lambda_{l}, 0\right)\right\rangle,
$$

and

$$
\rho^{l}(F(z))\left|\left(\lambda_{1}, \lambda_{2}, \ldots, \lambda_{l}\right)\right\rangle \neq \rho^{l+1}(F(z))\left|\left(\lambda_{1}, \lambda_{2}, \ldots, \lambda_{l}, 0\right)\right\rangle .
$$

To make it compatible with the inductive system, we need to define the action $\bar{\rho}$ : $U_{\mathfrak{q}, \mathfrak{o}}\left(\widehat{\mathfrak{g l}}_{1}\right) \rightarrow$ End $(\mathcal{F}(v))$ as follows: for each partition $\lambda=\left(\lambda_{1}, \lambda_{2}, \ldots, \lambda_{l}, 0,0, \ldots\right)$ we determine

$$
\begin{aligned}
\bar{\rho}\left(K^{ \pm}(z)\right)|\lambda\rangle & =\beta_{l+1}^{ \pm}\left((v / z)^{ \pm}\right) \cdot \rho^{l+1}\left(K^{ \pm}(z)\right)|\lambda\rangle, \\
\bar{\rho}(E(z))|\lambda\rangle & =\rho^{l+1}(E(z))|\lambda\rangle, \\
\bar{\rho}(F(z))|\lambda\rangle & =\beta_{l+1}^{+}(v / z) \rho^{l+1}(F(z))|\lambda\rangle,
\end{aligned}
$$


where

$$
\beta_{l}^{+}(v / z)=\psi\left(q_{1}^{-1} q_{2}^{l-1} v / z\right)^{-1} \quad \beta_{l}^{-}(z / v)=\psi\left(q_{2}^{-l} z / v\right) .
$$

This action is well-defined and compatible with the structure of inductive system. This is assured by the following theorem.

Theorem 3.4. Let $\lambda=\left(\lambda_{1}, \lambda_{2}, \ldots, \lambda_{l}, 0,0, \ldots\right)$ be a partition. Then, for any $k \in \mathbb{Z} \geq 1$

$$
\begin{aligned}
\bar{\rho}\left(K^{ \pm}(z)\right)|\lambda\rangle & =\beta_{l+k}^{ \pm}\left((v / z)^{ \pm}\right) \cdot \rho^{l+k}\left(K^{ \pm}(z)\right)|\lambda\rangle, \\
\bar{\rho}(E(z))|\lambda\rangle & =\rho^{l+k}(E(z))|\lambda\rangle, \\
\bar{\rho}(F(z))|\lambda\rangle & =\beta_{l+k}^{+}(v / z) \rho^{l+k}(F(z))|\lambda\rangle .
\end{aligned}
$$

Thus, we have equipped a $U_{\mathfrak{q}, \mathfrak{d}}\left(\widehat{\widehat{\mathfrak{g l}}}_{1}\right)$-module structure to $\mathcal{F}(v)$. We call $\left(\bar{\rho}: U_{\mathfrak{q}, \mathfrak{d}}\left(\widehat{\hat{\mathfrak{g}}}_{1}\right) \rightarrow\right.$ $\mathcal{F}(v), \mathcal{F}(v))$ the Fock representation of DIM algebra. The Fock representation is irreducible representation of level $(1, \mathfrak{q})[11,20]$.

\subsection{MacMahon representation}

Up to now we have constructed the Fock representation from the vector representations. Now we construct the MacMahon representation from the Fock representations by a similar process used in section 3.3.

Analogous to eq. (3.11), we define

$$
\mathcal{F}^{n}(v)=\mathcal{F}(v) \otimes \mathcal{F}\left(q_{3} v\right) \otimes \cdots \otimes \mathcal{F}\left(q_{3}^{n-1} v\right) .
$$

It is clear that the subset

$$
\left\{|\Lambda\rangle:=\left|\Lambda^{(1)}\right\rangle \otimes \cdots \otimes\left|\Lambda^{(n)}\right\rangle \mid \Lambda^{(1)}, \ldots, \Lambda^{(n)} \in \mathcal{P}^{+}\right\}
$$

forms a basis of $\mathcal{F}^{n}(v)$. Note that sometimes we write $\left|\Lambda^{(1)}, \ldots, \Lambda^{(n)}\right\rangle$ for $\left|\Lambda^{(1)}\right\rangle \otimes \cdots \otimes\left|\Lambda^{(n)}\right\rangle$.

Next we construct the subspace $\mathcal{M}^{n}(v)$ of $\mathcal{F}^{n}(v)$ spanned by plane partitions, i.e. the $\left|\Lambda^{(1)}, \ldots, \Lambda^{(n)}\right\rangle$ which satisfy the condition

$$
\Lambda_{i}^{(k)} \geq \Lambda_{i}^{(k+1)} \quad \forall i, k
$$

This step is analogous to eq. (3.16). Then, we collect these subspaces $\mathcal{M}^{n}(v)$ together by running $n$ over $\mathbb{Z}^{\geq 1}$, and then form an inductive system as (3.17).

Now we would like to endow a structure of $U_{\mathfrak{q}, \mathfrak{d}}\left(\widehat{\hat{\mathfrak{g}}}_{1}\right)$-module to the vector space $\lim _{n} \mathcal{M}^{n}(v)$ which is the inductive limit of the above-mentioned inductive system. As usual we first try to use the action as in the representation $\mathcal{F}^{n}(v)$. Again the problem arises: the action is not compatible with the structure of the inductive system. So we need a modification.

To make it compatible with the inductive system, we need to define the action $\bar{\varrho}$ : $U_{\mathfrak{q}, \mathfrak{d}}\left(\widehat{\mathfrak{g}}_{1}\right) \rightarrow$ End $\left(\lim _{\rightarrow n} \mathcal{M}^{n}(v)\right)$ as follows: for each $3 \mathrm{~d}$ partition $\Lambda=\left(\Lambda^{(1)}, \ldots, \Lambda^{(l)}, 0,0, \ldots\right)$ we determine

$$
\begin{aligned}
& \bar{\varrho}\left(K^{ \pm}(z)\right)|\Lambda\rangle=\gamma_{l+1}^{ \pm}\left((v / z)^{ \pm}\right) \cdot \varrho^{l+1}\left(K^{ \pm}(z)\right)|\Lambda\rangle, \\
& \bar{\varrho}(E(z))|\Lambda\rangle=\varrho^{l+1}(E(z))|\Lambda\rangle, \\
& \bar{\varrho}(F(z))|\Lambda\rangle=\gamma_{l+1}^{+}(v / z) \cdot \varrho^{l+1}(F(z))|\Lambda\rangle,
\end{aligned}
$$


where

$$
\gamma_{l}^{+}(v / z)=\frac{K^{-1 / 2}(1-K v / z)}{\mathfrak{q}^{-l}\left(1-q_{3}^{l} v / z\right)}, \quad \gamma_{l}^{-}(z / v)=\frac{K^{1 / 2}\left(1-\frac{z}{K v}\right)}{\mathfrak{q}^{l}\left(1-\frac{z}{q_{3}^{l} v}\right)} .
$$

Here $K$ is an arbitrary parameter which arises by the prescription of making the inductive system consistent [20]. The appearance of this continuous parameter $K$ is one of the most intriguing aspects of the MacMahon representation.

It is straightforward to check that the action $\bar{\varrho}$ is well-defined and compatible with the structure of the inductive system. Thus we have equipped the $U_{\mathfrak{q}, \mathfrak{d}}\left(\widehat{\widehat{\mathfrak{g l}}}_{1}\right)$-module structure to $\lim _{\rightarrow} \mathcal{M}^{n}(v)$. We call $\left(\bar{\varrho}: U_{\mathfrak{q}, \mathfrak{d}}\left(\widehat{\hat{\mathfrak{g l}}}_{1}\right) \rightarrow \operatorname{End}\left(\lim _{n} \mathcal{M}^{n}(v)\right), \lim _{n} \mathcal{M}^{n}(v)\right)$ the MacMahon module. It is easy to see that the MacMahon module has level $\left(1, K^{1 / 2}\right)$. Because of this, from now on we denote it by $\mathcal{M}(K ; v)$. As was shown in [15, 22], when $K=q_{1}^{a} q_{2}^{b} q_{3}^{c}, a, b, c \in$ $\mathbb{Z}_{\geq 0}$, the MacMahon representation is reducible. We can reduce the representation space to that spanned by plane partitons with a "pit" at $(a+1, b+1, c+1)$. In particular for $K=q_{3}$ the "pit" is at $(1,1,2)$ and the plane partition has only the first layer. Thus the representation is reduced to the Fock representation.

In the cases of the vector and the Fock representations, one of the parameters $\left(q_{1}, q_{2}, q_{3}\right)$ of the DIM algebra plays a distinguished role. Consequently there are three kinds of the vector and the Fock representations. ${ }^{6}$ On the other hand in the MacMahon representation three parameters are treated as an equal footing and the triality of DIM algebra is manifest.

\subsection{Horizontal Fock representation}

Up to now we only discussed about vertical representations. In this subsection we construct a horizontal representation of DIM algebra with $C \neq 1$. As in the case of the vector and the vertical Fock representations, there are three kinds of horizontal Fock representations, for which $C=q_{k}^{\frac{1}{2}}$. In the following we fix $k$ and write $\mathfrak{q}=q_{k}^{\frac{1}{2}}$. The conventional horizontal representation corresponds to the choice $k=3$. When $C=\mathfrak{q}$, from eq. (2.16) we obtain the Heisenberg algebra

$$
\left[H_{r}, H_{s}\right]=\delta_{r+s, 0} \frac{\kappa_{r}}{r}\left(\mathfrak{q}^{r}-\mathfrak{q}^{-r}\right) .
$$

There is a well-known representation of Heisenberg algebra whose representation space $\mathcal{F}$ is the Fock space of a (deformed) free boson with a creation operators $a_{-r}(r>0)$ acting on the vacuum state $|0\rangle$. The vacuum state is annihilated by the annihilation operator $a_{r}(r>0)$. Namely, we define the horizontal Fock representation by ${ }^{7}$

$$
\rho_{H}^{(\mathfrak{q}, 1)}\left(H_{r}\right):=\frac{\kappa_{r}}{r} a_{r}, \quad\left[a_{r}, a_{s}\right]=\delta_{r+s, 0} \frac{r}{\kappa_{r}}\left(\mathfrak{q}^{r}-\mathfrak{q}^{-r}\right) .
$$

\footnotetext{
${ }^{6}$ The conventional choice, which we follow in this paper, is to choose $q_{1}$ for the vertical representation and $q_{3}$ for the Fock representation. For the existence of the intertwiner we have to choose the parameters of the vector and the Fock representations differently.

${ }^{7}$ Since the only difference of $H_{r}$ and $a_{r}$ is the normalization, we will use them interchangeably in this paper.
} 
Now we try to endow $\mathcal{F}$ with the $U_{\mathfrak{q}, \mathfrak{d}}\left(\widehat{\hat{\mathfrak{g l}}}_{1}\right)$-module structure. We construct the action $\rho_{H}^{(\mathfrak{q}, 1)}: U_{\mathfrak{q}, \mathfrak{d}}\left(\widehat{\hat{\mathfrak{g l}}}_{1}\right) \rightarrow$ End $\mathcal{F}$ by

$$
\begin{aligned}
\rho_{H}^{(\mathfrak{q}, 1)}(E(z)) & =V^{-}\left(\mathfrak{q}^{-1 / 2} z\right) V^{+}\left(\mathfrak{q}^{1 / 2} z\right), \\
\rho_{H}^{(\mathfrak{q}, 1)}(F(z)) & =V^{-}\left(\mathfrak{q}^{1 / 2} z\right)^{-1} V^{+}\left(\mathfrak{q}^{-1 / 2} z\right)^{-1}, \\
\rho_{H}^{(\mathfrak{q}, 1)}\left(K^{ \pm}\left(\mathfrak{q}^{1 / 2} z\right)\right) & =V^{ \pm}\left(\mathfrak{q}^{ \pm 1} z\right) V^{ \pm}\left(\mathfrak{q}^{\mp 1} z\right)^{-1},
\end{aligned}
$$

where

$$
V^{ \pm}(z)=\exp \left(\mp \sum_{r=1}^{\infty} \frac{1}{r} \frac{\kappa_{r}}{\mathfrak{q}^{r}-\mathfrak{q}^{-r}} a_{ \pm r} z^{\mp r}\right)
$$

It is straightforward to show that $\left(\rho_{H}^{(\mathfrak{q}, 1)}, \mathcal{F}\right)$ is a representation of DIM algebra of level $(\mathfrak{q}, 1)$.

Now for a given $\gamma_{2} \in \mathbb{C} \backslash\{0\}$ we try to construct a horizontal representation of DIM algebra of level $\left(\mathfrak{q}, \gamma_{2}\right)$ by generalizing the above formula. While the representation space is the same as above, say $\mathcal{F}$, the action is modified to be $\rho_{H}^{\left(\mathfrak{q}, \gamma_{2}\right)}: U_{\mathfrak{q}, \mathfrak{o}}\left(\widehat{\mathfrak{g}}_{1}\right) \rightarrow$ End $\mathcal{F}$ by multiplying the zero mode factors $\mathbf{e}(z), \mathbf{f}(z), \mathbf{k}^{ \pm}(z)$;

$$
\begin{aligned}
\rho_{H}^{\left(\mathfrak{q}, \gamma_{2}\right)}(E(z)) & =\rho_{H}^{(\mathfrak{q}, 1)}(E(z)) \mathbf{e}(z), \\
\rho_{H}^{\left(\mathfrak{q}, \gamma_{2}\right)}(F(z)) & =\rho_{H}^{(\mathfrak{q}, 1)}(F(z)) \mathbf{f}(z), \\
\rho_{H}^{\left(\mathfrak{q}, \gamma_{2}\right)}\left(K^{ \pm}\left(\mathfrak{q}^{1 / 2} z\right)\right) & =\rho_{H}^{(\mathfrak{q}, 1)}\left(K^{ \pm}\left(\mathfrak{q}^{1 / 2} z\right)\right) \mathbf{k}^{ \pm}(z)
\end{aligned}
$$

where $\mathbf{e}(z), \mathbf{f}(z), \mathbf{k}^{ \pm}(z)$ should satisfy the condition

$$
\mathbf{e}(z) \mathbf{f}\left(\mathfrak{q}^{\mp 1} z\right)=\mathbf{k}^{ \pm}\left(\mathfrak{q}^{\mp 1 / 2} z\right)
$$

and

$$
\mathbf{k}^{ \pm}(z)=\mathbf{k}^{ \pm}(0)=\gamma_{2}^{\mp 1}
$$

It is straightforward to check that $\left(\rho_{H}^{\left(\mathfrak{q}, \gamma_{2}\right)}, \mathcal{F}\right)$ is a representation of DIM algebra of level $\left(\mathfrak{q}, \gamma_{2}\right)$.

When $\gamma_{2}=\mathfrak{q}^{N}$, by introducing the spectral parameter $u \in \mathbb{C}$ of the horizontal representation, we can solve the conditions (3.35) and (3.36) as follows;

$$
\mathbf{e}(z)=\left(\frac{\mathfrak{q}}{z}\right)^{N} u, \quad \mathbf{f}(z)=\left(\frac{\mathfrak{q}}{z}\right)^{-N} u^{-1}, \quad \mathbf{k}^{ \pm}(z)=\mathfrak{q}^{\mp N}
$$

which was originally employed in [2]. We denote the representation with this choice of $\mathbf{e}(z), \mathbf{f}(z)$ and $\mathbf{k}^{ \pm}(z)$ by $\mathcal{F}_{u}^{\left(\mathfrak{q}, \mathfrak{q}^{N}\right)}$, which is a representation of DIM algebra of level $\left(\mathfrak{q}, \mathfrak{q}^{N}\right)$. Recall that the MacMahon representation has a continuous parameter $K$ for the second level $\gamma_{2}$. Hence we can no longer use (3.37) when the MacMahon representation is involved. This is the reason why we need mode general zero mode algebra of $\mathbf{e}(z), \mathbf{f}(z), \mathbf{k}^{ \pm}(z)$. 


\section{Trivalent intertwiners}

In this section, we give a quick review on the trivalent intertwiners and the dual intertwiners. A trivalent intertwiner $\Psi: \mathcal{V} \otimes \mathcal{H} \rightarrow \mathcal{H}^{\prime}$, where $\mathcal{V}$ is a vertical representation and $\mathcal{H}, \mathcal{H}^{\prime}$ are horizontal representations, is determined by the intertwining relation

$$
a \Psi=\Psi \Delta(a) \quad \forall a \in U_{\mathfrak{q}, \mathfrak{d}}\left(\widehat{\hat{\mathfrak{g l}}}_{1}\right) .
$$

Taking a basis $\{\alpha\}$ of $\mathcal{V}$ which diagonalizes $H_{r}$, we define the $\alpha$-component of the intertwiner $\Psi_{\alpha}(\bullet)$ by

$$
\Psi_{\alpha}(\bullet)=\Psi(\alpha \otimes \bullet): \mathcal{H} \rightarrow \mathcal{H}^{\prime} .
$$

By using $\Psi_{\alpha}(\bullet)$ we can express the intertwining relation (4.1) as

$$
\begin{aligned}
K^{+}(z) \Psi_{\alpha} & =\left(\alpha\left|K^{+}(z)\right| \alpha\right) \Psi_{\alpha} K^{+}(z), \\
K^{-}(\mathfrak{q} z) \Psi_{\alpha} & =\left(\alpha\left|K^{-}(z)\right| \alpha\right) \Psi_{\alpha} K^{-}(\mathfrak{q} z), \\
E(z) \Psi_{\alpha} & =\sum_{\beta}(\beta|E(z)| \alpha) \Psi_{\beta}+\left(\alpha\left|K^{-}(z)\right| \alpha\right) \Psi_{\alpha} E(z), \\
F(z) \Psi_{\alpha} & =\sum_{\beta}(\beta|F(\mathfrak{q} z)| \alpha) \Psi_{\beta} K^{+}(\mathfrak{q} z)+\Psi_{\alpha} F(z) .
\end{aligned}
$$

When $\mathcal{V}$ is the vertical vector/Fock/MacMahon modules, we call the intertwiner $\Psi$ : $\mathcal{V} \otimes \mathcal{H} \rightarrow \mathcal{H}^{\prime}$ vector/Fock/MacMahon intertwiner, respectively. In the following we will summarize explicit expressions of these intertwiners which were derived in [11]. We also determine the dual intertwiners (see (4.28)-(4.31) for the definition) which were not given in [11].

\subsection{Vector intertwiner}

In terms of the basis $\left\{[v]_{n} \mid n \in \mathbb{Z}\right\}$ of $V(v)$ (see section 3.1), we define the components of the intertwiner by

$$
\mathbb{I}_{n}(v)(\bullet)=\mathbb{I}\left([v]_{n-1} \otimes \bullet\right): \mathcal{H} \rightarrow \mathcal{H}^{\prime} .
$$

A solution to the intertwining relations is

$$
\begin{aligned}
& \mathbb{I}_{n}(v)=z_{n} \tilde{\mathbb{I}}_{n}(v), \quad \tilde{\mathbb{I}}_{n}(v)=\tilde{\mathbb{I}}_{0}\left(q_{1}^{n} v\right), \quad n \in \mathbb{Z}, \\
& \tilde{\mathbb{I}}_{0}(v)=\exp \left(-\sum_{r=1}^{\infty} \frac{H_{-r}}{\mathfrak{q}^{r}-\mathfrak{q}^{-r}} \frac{\mathfrak{q}^{-r / 2}}{1-q_{1}^{r}} v^{r}\right) \exp \left(\sum_{r=1}^{\infty} \frac{H_{r}}{\mathfrak{q}^{r}-\mathfrak{q}^{-r}} \frac{\mathfrak{q}^{-r / 2}}{1-q_{1}^{-r}} v^{-r}\right) .
\end{aligned}
$$

Here

$$
z_{0}(v)=1, \quad z_{n}(v)=q_{2}^{-n} \prod_{j=1}^{n} \mathbf{e}\left(q_{1}^{j-1} v\right) \quad(n>0), \quad z_{n}(v)=q_{2}^{-n} \prod_{j=n}^{-1} \mathbf{e}\left(q_{1}^{j} v\right)^{-1} \quad(n<0) .
$$

For the existence of the intertwiner the zero modes $\mathbf{e}(z), \mathbf{f}(z), \mathbf{k}^{ \pm}(z)$ of $\mathcal{H}$ and $\mathbf{e}^{\prime}(z), \mathbf{f}^{\prime}(z), \mathbf{k}^{ \pm \prime}(z)$ of $\mathcal{H}^{\prime}$ have to be related by ${ }^{8}[21]$;

$$
\gamma_{2}^{\prime}=\gamma_{2}, \quad \mathbf{e}^{\prime}(z)=q_{2}^{-1} \mathbf{e}(z), \quad \mathbf{f}^{\prime}(z)=q_{2} \mathbf{f}(z) .
$$

From now on we are going to write $\gamma$ for $\gamma_{2}$ for the sake of convenience.

\footnotetext{
${ }^{8}$ Recall that $\mathbf{k}^{ \pm \prime}(z)=\gamma_{2}^{\mp 1}$.
} 


\subsection{Fock intertwiner}

Similarly for the set of partitions $\{|\lambda\rangle\}$ which forms a basis of $\mathcal{F}(v)$, the $\lambda$-component of the Fock intertwiner is defined by

$$
\Phi_{\lambda}(v)=\Phi(|\lambda\rangle \otimes \bullet): \mathcal{H} \rightarrow \mathcal{H}^{\prime} .
$$

A solution to the intertwining relations is

$$
\begin{aligned}
\Phi_{\lambda}(v)= & z_{\lambda} \mathcal{G}_{\lambda}^{-1} \exp \left(\sum_{r=1}^{\infty} \frac{H_{-r}}{\mathfrak{q}^{r}-\mathfrak{q}^{-r}} \mathfrak{q}^{-r / 2} v^{r}\left(\sum_{(i, j) \in \lambda} x_{i, j}^{r}-\frac{1}{\left(1-q_{1}^{r}\right)\left(1-q_{2}^{r}\right)}\right)\right) \\
& \times \exp \left(-\sum_{r=1}^{\infty} \frac{H_{r}}{\mathfrak{q}^{r}-\mathfrak{q}^{-r}} \mathfrak{q}^{-r / 2} v^{-r}\left(\sum_{(i, j) \in \lambda} x_{i, j}^{-r}-\frac{1}{\left(1-q_{1}^{-r}\right)\left(1-q_{2}^{-r}\right)}\right)\right),
\end{aligned}
$$

where $x_{i, j}=q_{1}^{j-1} q_{2}^{i-1}$. The zero mode factor is

$$
z_{\lambda}(v)=\prod_{i=1}^{\ell(\lambda)} \prod_{j=1}^{\lambda_{i}}\left(-\mathfrak{q} q_{2}^{i-1} x_{i, j}^{-1}\right) \mathbf{e}\left(x_{i, j} v\right)=q_{2}^{n(\lambda)}(-\mathfrak{q})^{|\lambda|} \prod_{(i, j) \in \lambda} x_{i, j}^{-1} \mathbf{e}\left(x_{i, j} v\right),
$$

and the normalization factor is

$$
\mathcal{G}_{\lambda}=\prod_{\square \in \lambda}\left(1-q_{1}^{-a_{\lambda}(\square)} q_{2}^{l_{\lambda}(\square)+1}\right) .
$$

The Fock intertwiner exists, if and only if the following relations between the zero modes of $\mathcal{H}$ and $\mathcal{H}^{\prime}$ are satisfied [2];

$$
\gamma^{\prime}=\mathfrak{q} \gamma, \quad \mathbf{e}^{\prime}(z)=(-\mathfrak{q} v / z) \mathbf{e}(z), \quad \mathbf{f}^{\prime}(z)=(-\mathfrak{q} v / z)^{-1} \mathbf{f}(z) .
$$

Compared with (3.37), this means that the level and the spectral parameter are shifted by $\mathfrak{q}^{N} \rightarrow \mathfrak{q}^{N+1}$ and $u \rightarrow-u v$.

\subsection{MacMahon intertwiner}

Now let's consider the MacMahon intertwiner whose vertical representation is $\mathcal{M}(K ; v)$. We know that the set of plane partitions $\{|\Lambda\rangle\}$ forms a basis of $\mathcal{M}(K ; v)$. We define the $\Lambda$-component of the MacMahon intertwiner by

$$
\Xi_{\Lambda}(K ; v)=\Xi(|\Lambda\rangle \otimes \bullet): \mathcal{H} \rightarrow \mathcal{H}^{\prime} .
$$

A solution to the intertwining relations is

$$
\Xi_{\Lambda}(K ; v)=z_{\Lambda}(K ; v) \mathcal{M}^{[n]}(K) \tilde{\Phi}_{\Lambda}^{[n]}(v) \Gamma_{n}(K ; v), \quad n>h(\Lambda),
$$

where

$$
\Gamma_{n}(K ; v)=\exp \left(\sum_{r=1}^{\infty} \frac{H_{-r}}{\mathfrak{q}^{r}-\mathfrak{q}^{-r}} \frac{q_{3}^{n r}-K^{r}}{\kappa_{r}} \mathfrak{q}^{-r / 2} v^{r}\right) \exp \left(\sum_{r=1}^{\infty} \frac{H_{r}}{\mathfrak{q}^{r}-\mathfrak{q}^{-r}} \frac{q_{3}^{-n r}-K^{-r}}{\kappa_{r}} \mathfrak{q}^{-r / 2} v^{-r}\right)
$$


is the vacuum contribution which survives for $\Lambda=\varnothing$. The zero mode factor is

$$
z_{\Lambda}(K ; v)=\prod_{k=1}^{h(\Lambda)} \prod_{(i, j) \in \Lambda^{(k)}} \frac{K^{1 / 2}}{\mathfrak{q}^{k-1}} \frac{\theta_{q_{3}}\left(q_{3}^{k-1} / x_{i j k}\right)}{\theta_{q_{3}}\left(K / x_{i j k}\right)} \mathbf{e}\left(x_{i j k} v\right),
$$

where $x_{i j k}=q_{1}^{i} q_{2}^{j} q_{3}^{k}$. Note that for later convenience we have exchanged $q_{1}$ and $q_{2}$ in the original formula in [11]. This is equivalent to taking the transpose of the Young diagram $\lambda$ which labels the basis of the Fock representation. Or we may exchange the role of $q_{1}$ and $q_{2}$ in the construction of the Fock representation in section 3.3. Namely we begin with the vector representation $\rho^{V^{(2)}}$ and choose $q_{1}$ as the shift parameter in the tensor product. Furthermore,

$$
\tilde{\Phi}_{\Lambda}^{[n]}(v)=\tilde{\Phi}_{\Lambda^{(1)}}(v) \circ \cdots \circ \tilde{\Phi}_{\Lambda^{(n)}}\left(q_{3}^{n-1} v\right)
$$

is a composition of the Fock intertwiners $\tilde{\Phi}$ without the zero mode factor. Finally the factor $\mathcal{M}^{[n]}(K)$ is defined via

$$
: \tilde{\Phi}_{\varnothing}^{[n]}(v) \Gamma_{n}(K ; v):=\mathcal{M}^{[n]}(K) \tilde{\Phi}_{\varnothing}^{[n]}(v) \Gamma_{n}(K ; v) .
$$

The notation $n>h(\Lambda)$ in eq. (4.18) means that we can choose any integer $n$ which is greater than $h(\Lambda)$. All of them give the same $\Xi_{\Lambda}(K ; v)$. It can be shown that

$$
\begin{aligned}
\Xi_{\Lambda}(K ; v)= & z_{\Lambda} \mathcal{G}_{\Lambda}^{-1} \exp \left(\sum_{r=1}^{\infty} \frac{H_{-r}}{\mathfrak{q}^{r}-\mathfrak{q}^{-r}} \mathfrak{q}^{-r / 2} v^{r}\left(\sum_{(i, j, k) \in \Lambda} x_{i j k}^{r}+\frac{1-K^{r}}{\kappa_{r}}\right)\right) \\
& \times \exp \left(-\sum_{r=1}^{\infty} \frac{H_{r}}{\mathfrak{q}^{r}-\mathfrak{q}^{-r}} \mathfrak{q}^{-r / 2} v^{-r}\left(\sum_{(i, j, k) \in \Lambda} x_{i j k}^{-r}-\frac{1-K^{-r}}{\kappa_{r}}\right)\right),
\end{aligned}
$$

where the factor $\mathcal{G}_{\Lambda}$ is defined by

$$
: \tilde{\Phi}_{\Lambda}^{[n]}(v) \Gamma_{n}(K ; v):=\mathcal{G}_{\Lambda} \mathcal{M}^{[n]}(K) \tilde{\Phi}_{\Lambda}^{[n]}(v) \Gamma_{n}(K ; v) .
$$

As motivated from the Fock intertwiners, we can construct the MacMahon intertwiner from the Fock intertwiners by composing them together with the correction intertwiner [11]. From this method of construction, we obtain the explicit form of $\mathcal{G}_{\Lambda}$. Nevertheless, in this paper, the explicit form is not relevant and we do not state it here. We obtain the following relations between the zero modes of $\mathcal{H}$ and $\mathcal{H}^{\prime}$

$$
\gamma^{\prime}=K^{1 / 2} \gamma, \quad \mathbf{e}^{\prime}(z)=K^{1 / 2} \frac{\theta_{q_{3}}(v / z)}{\theta_{q_{3}}(K v / z)} \mathbf{e}(z), \quad \mathbf{f}^{\prime}(z)=\frac{\theta_{q_{3}}(\mathfrak{q} K v / z)}{\theta_{q_{3}}(\mathfrak{q} v / z)} \mathbf{f}(z)
$$

Note that when $K=q_{3},(4.25)$ reduces to (4.16).

\subsection{Dual intertwiners}

Now we turn to the construction of the dual intertwiner $\Psi^{*}: \mathcal{H}^{\prime} \rightarrow \mathcal{H} \otimes \mathcal{V}$, which is determined from the intertwining relation:

$$
\Delta(a) \Psi^{*}=\Psi^{*} a, \quad \forall a \in U_{\mathfrak{q}, \mathfrak{d}}\left(\widehat{\mathfrak{g}}_{1}\right) .
$$


Taking the same basis $\{\alpha\}$ of $\mathcal{V}$ used to define the components of $\Psi$, we can define the $\alpha$-component of the dual intertwiner $\Psi_{\alpha}^{*}: \mathcal{H}^{\prime} \rightarrow \mathcal{H}$ by

$$
\Psi^{*}(\bullet)=\sum_{\alpha} \Psi_{\alpha}^{*}(\bullet) \otimes|\alpha\rangle, \quad \bullet \in \mathcal{H}^{\prime}
$$

We can express the intertwining relation (4.26) in terms of the $\alpha$-component as

$$
\begin{aligned}
\Psi_{\alpha}^{*} K^{+}(\mathfrak{q} z) & =\left(\alpha\left|K^{+}(z)\right| \alpha\right) K^{+}(\mathfrak{q} z) \Psi_{\alpha}^{*}, \\
\Psi_{\alpha}^{*} K^{-}(z) & =\left(\alpha\left|K^{-}(z)\right| \alpha\right) K^{-}(z) \Psi_{\alpha}^{*}, \\
\Psi_{\alpha}^{*} E(z) & =K^{-}(\mathfrak{q} z) \sum_{\beta}(\alpha|E(\mathfrak{q} z)| \beta) \Psi_{\beta}^{*}+E(z) \Psi_{\alpha}^{*}, \\
\Psi_{\alpha}^{*} F(z) & =\sum_{\beta}(\alpha|F(z)| \beta) \Psi_{\beta}^{*}+\left(\alpha\left|K^{+}(z)\right| \alpha\right) F(z) \Psi_{\alpha}^{*} .
\end{aligned}
$$

When $\mathcal{V}$ is the vertical vector/Fock/MacMahon modules, we call the dual intertwiner $\Psi^{*}$ : $\mathcal{H}^{\prime} \rightarrow \mathcal{H} \otimes \mathcal{V}$ the vector/Fock/MacMahon dual intertwiner, respectively. In the following we give their explicit expressions in each case.

We know that $\left\{[v]_{n} \mid n \in \mathbb{Z}\right\}$ forms a basis of $V(v)$. Then, according to (4.27), the $n$-component $\mathbb{I}_{n}^{*}(v): \mathcal{H}^{\prime} \rightarrow \mathcal{H}$ of the dual intertwiner satisfies

$$
\mathbb{I}^{*}(v)=\sum_{n \in \mathbb{Z}} \mathbb{I}_{n}^{*}(v) \otimes[v]_{n-1} .
$$

Thus, the intertwining relations become

$$
\begin{aligned}
\mathbb{I}_{n}^{*}(v) K^{+}(\mathfrak{q} z) & =\tilde{\psi}\left(q_{1}^{n-1} v / z\right) K^{+}(\mathfrak{q} z) \mathbb{I}_{n}^{*}(v), \\
\mathbb{I}_{n}^{*}(v) K^{-}(z) & =\tilde{\psi}\left(q_{1}^{-n} z / v\right) K^{-}(z) \mathbb{I}_{n}^{*}(v), \\
\mathbb{I}_{n}^{*}(v) E(z) & =\left(1-q_{1}\right)^{-1} \delta\left(\mathfrak{q}^{-1} q_{1}^{n-1} v / z\right) K^{-}(\mathfrak{q} z) \mathbb{I}_{n-1}^{*}(v)+E(z) \mathbb{I}_{n}^{*}(v), \\
\mathbb{I}_{n}^{*}(v) F(z) & =\left(1-q_{1}^{-1}\right)^{-1} \delta\left(q_{1}^{n} v / z\right) \mathbb{I}_{n+1}^{*}(v)+\tilde{\psi}\left(q_{1}^{n-1} v / z\right) F(z) \mathbb{I}_{n}^{*}(v) .
\end{aligned}
$$

A solution to the intertwining relations is

$$
\mathbb{I}_{n}^{*}(v)=z_{n}^{*} \exp \left(\sum_{r=1}^{\infty} \frac{H_{-r}}{\mathfrak{q}^{r}-\mathfrak{q}^{-r}} \frac{\mathfrak{q}^{r / 2}\left(q_{1}^{n} v\right)^{r}}{1-q_{1}^{r}}\right) \exp \left(-\sum_{r=1}^{\infty} \frac{H_{r}}{\mathfrak{q}^{r}-\mathfrak{q}^{-r}} \frac{\mathfrak{q}^{r / 2}\left(q_{1}^{n} v\right)^{-r}}{1-q_{1}^{-r}}\right)
$$

where

$$
z_{n}^{*}= \begin{cases}q_{2}^{n} \prod_{j=1}^{n} \mathbf{f}\left(q_{1}^{j-1} v\right) & ; n>0, \\ q_{2}^{n} \prod_{j=n}^{-1} \mathbf{f}\left(q_{1}^{j} v\right)^{-1} & ; n<0 .\end{cases}
$$

As the condition for the existence of the dual intertwiner, we get the following relations [21];

$$
\gamma^{\prime}=\gamma, \quad \mathbf{e}^{\prime}(z)=q_{2}^{-1} \mathbf{e}(z), \quad \mathbf{f}^{\prime}(z)=q_{2} \mathbf{f}(z) .
$$

We define the $\lambda$-component of the dual Fock intertwiner $\Phi_{\lambda}^{*}(v)$ by

$$
\Phi^{*}(v)=\sum_{\lambda} \Phi_{\lambda}^{*}(v) \otimes|\lambda\rangle .
$$


A solution to the dual intertwining relations is

$$
\Phi_{\lambda}^{*}(v)=z_{\lambda}^{*}(v) \mathcal{G}^{[n] *} B_{n}^{*}(v) \tilde{\mathbb{I}}_{\lambda}^{[n] *}(v), \quad n>\ell(\lambda),
$$

where

$$
B_{n}^{*}(v)=\exp \left(\sum_{r=1}^{\infty} \frac{1}{k_{r}}\left(\mathfrak{q}^{3 / 2} q_{2}^{n} v\right)^{r} H_{-r}\right) \exp \left(-\sum_{r=1}^{\infty} \frac{1}{\kappa_{r}}\left(\mathfrak{q}^{1 / 2} q_{2}^{n} v\right)^{-r} H_{r}\right),
$$

$\tilde{\mathbb{I}}_{\lambda}^{[n] *}:=\tilde{\mathbb{I}}_{\lambda_{n}}^{*}\left(q_{2}^{n-1} v\right) \circ \cdots \circ \tilde{\mathbb{I}}_{\lambda_{1}}^{*}(v)$, and the normalization factor $\mathcal{G}^{[n] *}$ is defined by : $B_{n}^{*}(v) \tilde{\mathbb{I}}_{\emptyset}^{[n] *}(v):=\mathcal{G}^{[n] *} B_{n}^{*}(v) \tilde{\mathbb{I}}_{\emptyset}^{[n] *}(v)$. The factor $z_{\lambda}^{*}(v)$ is

$$
z_{\lambda}^{*}(v)=\mathfrak{q}^{|\lambda|} \prod_{i=1}^{l(\lambda)} \prod_{j=1}^{\lambda_{i}}\left(-\mathfrak{q} q_{2}^{i-1} x_{i, j}^{-1}\right)^{-1} \mathbf{f}\left(x_{i j} v\right) .
$$

It can also be shown that

$$
\begin{aligned}
\Phi_{\lambda}^{*}(v)= & z_{\lambda}^{*} \mathcal{G}_{\lambda}^{*-1} \exp \left[\sum_{r=1}^{\infty} \frac{H_{-r}}{\mathfrak{q}^{r}-\mathfrak{q}^{-r}} \mathfrak{q}^{r / 2} v^{r}\left(\frac{q_{2}^{l r}}{\left(1-q_{1}^{r}\right)\left(1-q_{2}^{r}\right)}+\sum_{k=1}^{l} \frac{\left(q_{1}^{\lambda_{k}} q_{2}^{k-1}\right)^{r}}{1-q_{1}^{r}}\right)\right] \\
& \times \exp \left[\sum_{r=1}^{\infty} \frac{H_{r}}{\mathfrak{q}^{r}-\mathfrak{q}^{-r}} \mathfrak{q}^{r / 2} v^{-r}\left(\sum_{k=1}^{l} \frac{\left(q_{1}^{\lambda_{k}-1} q_{2}^{k-1}\right)^{-r}}{1-q_{1}^{r}}-\frac{q_{2}^{-r l}}{\left(1-q_{1}^{-r}\right)\left(1-q_{2}^{-r}\right)}\right)\right] .
\end{aligned}
$$

Here $\mathcal{G}_{\lambda}^{*}$ is defined via the equation

$$
: B_{n}^{*}(v) \tilde{\mathbb{I}}_{\lambda}^{[n] *}(v):=\mathcal{G}_{\lambda}^{*} \mathcal{G}^{[n] *} B_{n}^{*}(v) \tilde{\mathbb{I}}_{\lambda}^{[n] *}(v) .
$$

Similarly we can define the $\Lambda$-component of the MacMahon intertwiner $\Xi_{\Lambda}^{*}(K, v)$ by

$$
\Xi^{*}(K ; v)=\sum_{\Lambda} \Xi_{\Lambda}^{*}(K ; v) \otimes|\Lambda\rangle .
$$

A solution to the intertwining relations is

$$
\Xi_{\Lambda}^{*}(K ; v)=z_{\Lambda}^{*}(K ; v) \mathcal{M}^{[n] *}(K) \tilde{\Phi}_{\Lambda}^{[n] *}(v) \Gamma_{n}^{*}(K ; v),
$$

where

$$
\begin{aligned}
\Gamma_{n}^{*}(K ; v)= & \exp \left(-\sum_{r=1}^{\infty} \frac{H_{-r}}{\mathfrak{q}^{r}-\mathfrak{q}^{-r}} \frac{q_{3}^{n r}-K^{r}}{\kappa_{r}} \mathfrak{q}^{r / 2} v^{r}\right) \\
& \times \exp \left(-\sum_{r=1}^{\infty} \frac{H_{r}}{\mathfrak{q}^{r}-\mathfrak{q}^{-r}} \frac{q_{3}^{-n r}-K^{-r}}{\kappa_{r}} \mathfrak{q}^{r / 2} v^{-r}\right), \\
z_{\Lambda}^{*}(K ; v)= & \prod_{k=1}^{h(\Lambda)} \prod_{(i, j) \in \Lambda^{(k)}}\left(\frac{K^{1 / 2}}{\mathfrak{q}^{k}} \frac{\theta_{q_{3}}\left(q_{3}^{k} x_{i j k}^{-1}\right)}{\theta_{q_{3}}\left(K x_{i j k}^{-1}\right)}\right)^{-1} \mathbf{f}\left(x_{i j k} v\right),
\end{aligned}
$$

and

$$
\tilde{\Phi}_{\Lambda}^{[n] *}(v)=\tilde{\Phi}_{\Lambda^{(1)}}^{*}(v) \circ \cdots \circ \tilde{\Phi}_{\Lambda^{(n)}}^{*}\left(q_{3}^{n-1} v\right) .
$$

The factor $\mathcal{M}^{[n] *}(K)$ is defined by the equation

$$
\text { : } \tilde{\Phi}_{\emptyset}^{[n] *}(v) \Gamma_{n}^{*}(K ; v):=\mathcal{M}^{[n] *}(K) \tilde{\Phi}_{\emptyset}^{[n] *}(v) \Gamma_{n}^{*}(K ; v) .
$$

As in the case of the dual Fock intertwiner, we can determine an explicit form of $\Xi_{\Lambda}^{*}(K ; v)$ like eq. (4.44), which we will compute in section 6 . 


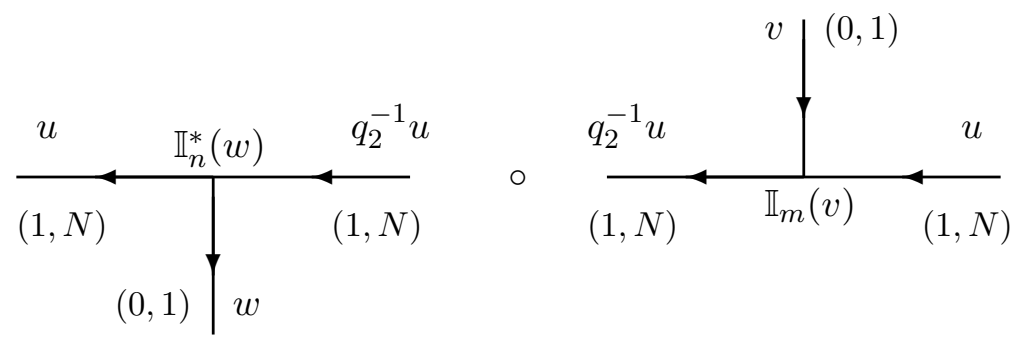

Figure 1. Composition o of the vector intertwiner and the dual vector intertwiner. Contrary to the Fock intertwiner, the level $(1, N)$ of the horizontal Fock space is kept intact and the shift of the spectral parameter $u$ is independent of the spectral parameters $v, w$ of the vertical representation.

\section{Generalized $\mathrm{KZ}$ equation for the vector intertwiners}

In this section we derive the generalized $\mathrm{KZ}$ equation for the vector intertwiners as a warmup exercise before attacking the more complicated case of the MacMahon intertwiners. The first step is to construct the shift operator $\mathcal{T}$.

\subsection{Construction of the shift operator}

In [9] the shift operator is constructed as the composition of the intertwiner and the dual intertwiner with appropriate spectral parameter. We first recall the relation (4.11) on the level of the vector intertwiner discussed in the previous section. Comparing the choice of the zero modes (3.37) with (4.11) saying there is no change of the level of $\mathcal{H}$ and $\mathcal{H}^{\prime}$, we see $u^{\prime}=q_{2}^{-1} u$. We define the operator $\mathcal{T}_{n}^{m}$ by the composition of the intertwiner and the dual intertwiner;

$$
\mathcal{T}_{n}^{m}(N, u \mid v, w):=\mathbb{I}_{n}^{*}(w) \mathbb{I}_{m}(v) \quad: \quad \mathcal{F}_{u}^{\left(\mathfrak{q}, \mathfrak{q}^{N}\right)} \longrightarrow \mathcal{F}_{u}^{\left(\mathfrak{q}, \mathfrak{q}^{N}\right)} .
$$

Pictorially, it is represented as figure 1.

Using the commutation relation (3.30), we see that

$$
\begin{aligned}
\mathcal{T}_{n}^{m}(N, u \mid v, w)= & z_{n}^{*}(u, N \mid w) z_{m}(u, N \mid v) \\
& \cdot \exp \left(-\sum_{r=1}^{\infty} \frac{q_{1}^{-r(n-1)} w^{-r}\left(q_{1}^{m} v\right)^{r}}{r} \frac{\mathfrak{q}^{r}\left(1-q_{2}^{r}\right)}{1-q_{1}^{r}}\right): \tilde{\mathbb{I}}_{n}^{*}(w) \tilde{\mathbb{I}}_{m}(v):,
\end{aligned}
$$

where : : denotes the normal ordering. The next step is to determine the value of the shift parameter $p$ such that we can express $\mathbb{I}_{n}(p v)$ and $\mathbb{I}_{n}^{*}(p v)$ by the action of $\mathcal{T}$ operator. Consider the quantity $\mathbb{I}_{n}(p v)$. From the explicit expression of the vector intertwiner explained in section 4.1 , we see that

$$
\begin{aligned}
\mathbb{I}_{n}(p v)= & \frac{z_{n}(u, N \mid p v)}{z_{n}(u, N \mid v)} \cdot \exp \left(-\sum_{r=1}^{\infty} \frac{H_{-r}}{\mathfrak{q}^{r}-\mathfrak{q}^{-r}} \frac{\left(\mathfrak{q}^{-1 / 2} q_{1}^{n} v\right)^{r}}{1-q_{1}^{r}}\left(p^{r}-1\right)\right) \\
& \cdot \mathbb{I}_{n}(v) \cdot \exp \left(\sum_{r=1}^{\infty} \frac{H_{r}}{\mathfrak{q}^{r}-\mathfrak{q}^{-r}} \frac{\left(\mathfrak{q}^{1 / 2} q_{1}^{n-1} v\right)^{-r}}{1-q_{1}^{r}}\left(1-p^{-r}\right)\right) .
\end{aligned}
$$


To express the exponential factors in (5.3) by $\mathcal{T}$ operator, let us consider eq. (5.2) in the case $m=n$.

$$
\begin{aligned}
\mathcal{T}_{n}^{n}(N, u \mid v, w)= & z_{n}^{*}(u, N \mid w) z_{n}(u, N \mid v) \exp \left(-\sum_{r=1}^{\infty} \frac{q_{1}^{r} w^{-r} v^{r}}{r} \frac{\mathfrak{q}^{r}\left(1-q_{2}^{r}\right)}{1-q_{1}^{r}}\right) \\
& \cdot \exp \left(\sum_{r=1}^{\infty} \frac{H_{-r}}{\left(\mathfrak{q}^{r}-\mathfrak{q}^{-r}\right)\left(1-q_{1}^{r}\right)}\left(\left(\mathfrak{q}^{1 / 2} q_{1}^{n} w\right)^{r}-\left(\mathfrak{q}^{-1 / 2} q_{1}^{n} v\right)^{r}\right)\right) \\
& \cdot \exp \left(\sum_{r=1}^{\infty} \frac{H_{r}}{\left(\mathfrak{q}^{r}-\mathfrak{q}^{-r}\right)\left(1-q_{1}^{r}\right)}\left(\left(\mathfrak{q}^{-1 / 2} q_{1}^{n-1} w\right)^{-r}-\left(\mathfrak{q}^{1 / 2} q_{1}^{n-1} v\right)^{-r}\right)\right)
\end{aligned}
$$

Thus, if $w=\mathfrak{q} v$, the positive modes disappear

$$
\mathcal{T}_{n}^{n}(N, u \mid v, \mathfrak{q} v)=\exp \left(-\sum_{r=1}^{\infty} \frac{q_{1}^{r}}{r} \frac{1-q_{2}^{r}}{1-q_{1}^{r}}\right) \cdot \exp \left(\sum_{r=1}^{\infty} \frac{H_{-r} \mathfrak{q}^{r / 2}}{1-q_{1}^{r}}\left(q_{1}^{n} v\right)^{r}\right) .
$$

where we have used $z_{n}^{*}\left(u, N \mid \mathfrak{q}^{ \pm 1} v\right) z_{n}(u, N \mid v)=1$ for $\gamma=1$. On the other hand, if $w=$ $\mathfrak{q}^{-1} v$, the negative modes disappear

$$
\mathcal{T}_{n}^{n}\left(N, u \mid v, \mathfrak{q}^{-1} v\right)=\exp \left(-\sum_{r=1}^{\infty} \frac{q_{1}^{r} \mathfrak{q}^{2 r}\left(1-q_{2}^{r}\right)}{r\left(1-q_{1}^{r}\right)}\right) \cdot \exp \left(\sum_{r=1}^{\infty} \frac{H_{r} \mathfrak{q}^{r / 2}}{1-q_{1}^{r}}\left(q_{1}^{n-1} v\right)^{-r}\right)
$$

By tuning the spectral parameters of $\mathbb{I}_{n}^{*}(w)$ and $\mathbb{I}_{m}(v)$, we define ${ }^{9}$

$$
\begin{aligned}
& \mathcal{T}_{n}^{+}(N, u \mid v):=\mathcal{T}_{n}^{n}\left(N, u \mid \mathfrak{q}^{1 / 2} v, \mathfrak{q}^{-1 / 2} v\right)^{-1} \\
& \mathcal{T}_{n}^{-}(N, u \mid v):=\mathcal{T}_{n}^{n}\left(N, u \mid \mathfrak{q}^{-1 / 2} v, \mathfrak{q}^{1 / 2} v\right) .
\end{aligned}
$$

Thus,

$$
\begin{aligned}
\mathbb{I}_{n}\left(\mathfrak{q}^{-2} v\right)= & \frac{z_{n}\left(u, N \mid \mathfrak{q}^{-2} v\right)}{z_{n}(u, N \mid v)} \cdot \exp \left(\sum_{r=1}^{\infty} \frac{q_{1}^{r}}{r} \frac{1-q_{2}^{r}}{1-q_{1}^{r}}\left(1-\mathfrak{q}^{2 r}\right)\right) \\
& \cdot \mathcal{T}_{n}^{-}\left(N, q_{2}^{-1} u \mid \mathfrak{q}^{-3 / 2} v\right) \cdot \mathbb{I}_{n}(v) \cdot \mathcal{T}_{n}^{+}\left(N, u \mid \mathfrak{q}^{-1 / 2} v\right)
\end{aligned}
$$

It is remarkable that the shift parameter is fixed to be $p=\mathfrak{q}^{-2}$. In the above tuning the spectral parameters of the intertwiners and the dual intertwiners are shifted by $\mathfrak{q}^{ \pm 1}$. This reminds us of exactly the same shift of the spectral parameters in the "Higgsed" DIM network $[24,25,42,46] .{ }^{10}$ It seems that this coincidence is related to the fact the shift operator (5.7) is obtained from the universal $R$ matrix as we show later in section 5.5.

Similarly from the explicit expression of the dual vector intertwiner explained in section 4.4 , we see that

$$
\begin{aligned}
\mathbb{I}_{n}^{*}(p v)= & \frac{z_{n}^{*}(u, N \mid p v)}{z_{n}^{*}(u, N \mid v)} \cdot \exp \left[\sum_{r=1}^{\infty} \frac{H_{-r}}{\mathfrak{q}^{r}-\mathfrak{q}^{-r}} \frac{\mathfrak{q}^{r / 2}\left(q_{1}^{n} v\right)^{r}}{1-q_{1}^{r}}\left(p^{r}-1\right)\right] \\
& \cdot \mathbb{I}_{n}^{*}(v) \cdot \exp \left[-\sum_{r=1}^{\infty} \frac{H_{r}}{\mathfrak{q}^{r}-\mathfrak{q}^{-r}} \frac{\mathfrak{q}^{r / 2}\left(q_{1}^{n} v\right)^{-r}}{1-q_{1}^{-r}}\left(p^{-r}-1\right)\right] .
\end{aligned}
$$

\footnotetext{
${ }^{9}$ See section 5.5 for the reason why we define them in this way.

${ }^{10}$ Upon the completion of the present work, we noticed [47], which shows the universal $R$ matrix of DIM algebra plays a significant role in the "Higgsed" network calculus. In particular it is interesting that the shift operator in the present paper is physically identified with the crossing of branes in [47].
} 


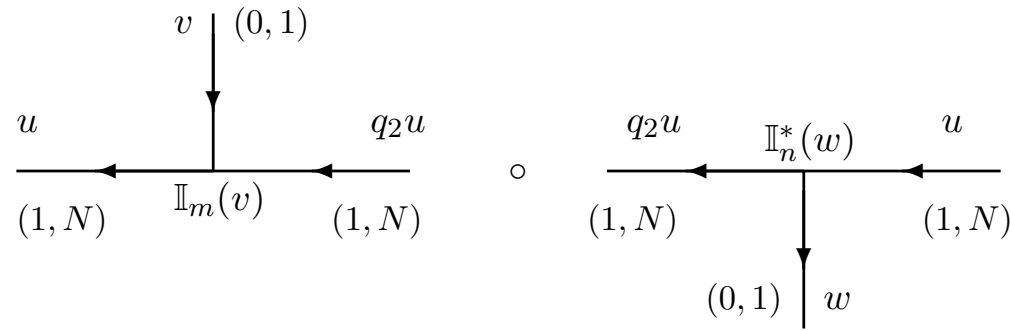

Figure 2. Composition o of the dual vector intertwiner and the vector intertwiner. Contrary to the Fock intertwiner, the level $(1, N)$ of the horizontal Fock space is kept intact and the shift of the spectral parameter $u$ is independent of the spectral parameters $v, w$ of the vertical representation.

From eqs. (5.5) and (5.6), we get that

$$
\begin{aligned}
\mathbb{I}_{n}^{*}\left(\mathfrak{q}^{-2} v\right)= & \frac{z_{n}^{*}\left(u, N \mid \mathfrak{q}^{-2} v\right)}{z_{n}^{*}(u, N \mid v)} \cdot \exp \left[\sum_{r=1}^{\infty} \frac{q_{1}^{r}\left(1-q_{2}^{r}\right)}{r\left(1-q_{1}^{r}\right)}\left(\mathfrak{q}^{2 r}-1\right)\right] \\
& \cdot \mathcal{T}_{n}^{-}\left(N, q_{2} u \mid \mathfrak{q}^{-1 / 2} v\right)^{-1} \cdot \mathbb{I}_{n}^{*}(v) \cdot \mathcal{T}_{n}^{+}\left(N, u \mid \mathfrak{q}^{-3 / 2} v\right)^{-1} .
\end{aligned}
$$

\subsection{Commutation of vector intertwiner and the shift operators}

From the previous subsection, we see that $\mathcal{T}$ operators are expressed as the composition of the intertwiner and the dual vector intertwiner. Thus, to calculate the commutation of the vector intertwiner and $\mathcal{T}$ operators, we first calculate the commutation between the vector intertwiners and the dual vector intertwiners themselves.

The result of the composition $\mathbb{I}_{n}^{*}(w) \mathbb{I}_{m}(v)$ of figure 1 has already given in eq. (5.2). On the other hand the composition $\mathbb{I}_{m}(v) \mathbb{I}_{n}^{*}(w)$ of figure 2 is

$$
\begin{aligned}
\mathbb{I}_{m}(v) \mathbb{I}_{n}^{*}(w)= & z_{m}\left(q_{2} u, N \mid v\right) z_{n}^{*}\left(q_{2} u, N \mid w\right) \\
& \cdot \exp \left(-\sum_{r=1}^{\infty} \frac{1}{r}\left(\mathfrak{q}^{1 / 2} q_{1}^{m-1} v\right)^{-r}\left(\mathfrak{q}^{1 / 2} q_{1}^{n} w\right)^{r} \mathfrak{q}^{r} \frac{1-q_{2}^{r}}{1-q_{1}^{r}}\right): \tilde{\mathbb{I}}_{m}(v) \tilde{\mathbb{I}}_{n}^{*}(w): .
\end{aligned}
$$

Since

$$
z_{m}\left(q_{2} u, N \mid v\right)=q_{2}^{m} z_{m}(u, N \mid v), \quad z_{n}^{*}\left(q_{2} u, N \mid w\right)=q_{2}^{-n} z_{n}^{*}(u, N \mid w),
$$

we get that

$$
\mathbb{I}_{m}(v) \mathbb{I}_{n}^{*}(w)=q_{2}^{m-n} \exp \left(\sum_{r=1}^{\infty} \frac{q_{1}^{r} \mathfrak{q}^{r}}{r} \frac{1-q_{2}^{r}}{1-q_{1}^{r}}\left[q_{1}^{-r n} q_{1}^{m r}(v / w)^{r}-q_{1}^{-m r} q_{1}^{n r}(w / v)^{r}\right]\right) \mathbb{I}_{n}^{*}(w) \mathbb{I}_{m}(v) .
$$

Next let us calculate $\mathbb{I}_{m}\left(v^{\prime}\right) \mathbb{I}_{n}(v)$ of figure 3 . The result is

$$
\begin{aligned}
\mathbb{I}_{m}\left(v^{\prime}\right) \mathbb{I}_{n}(v)= & z_{m}\left(q_{2}^{-1} u, N \mid v^{\prime}\right) z_{n}(u, N \mid v) \\
& \cdot \exp \left(\sum_{r=1}^{\infty}\left(q_{1}^{m-1} v^{\prime}\right)^{-r}\left(q_{1}^{n} v\right)^{r} \frac{1}{r} \cdot \frac{1-q_{2}^{r}}{1-q_{1}^{r}}\right): \tilde{\mathbb{I}}_{m}\left(v^{\prime}\right) \tilde{\mathbb{I}}_{n}(v): .
\end{aligned}
$$



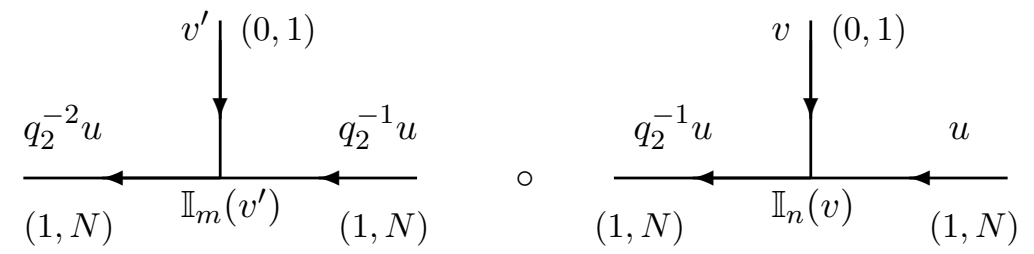

Figure 3. Composition $\circ$ of the vector intertwiner and the vector intertwiner. Contrary to the Fock intertwiner, the level $(1, N)$ of the horizontal Fock space is kept intact and the shift of the spectral parameter $u$ is independent of the spectral parameters $v, v^{\prime}$ of the vertical representation.

The formula for $\mathbb{I}_{n}(v) \mathbb{I}_{m}\left(v^{\prime}\right)$ is obtained by interchanging the parameters $v \leftrightarrow v^{\prime}$, and $n \leftrightarrow m$. Hence, by (5.11), we see that

$$
\mathbb{I}_{n}(v) \mathbb{I}_{m}\left(v^{\prime}\right)=q_{2}^{-n+m} \exp \left(\sum_{r=1}^{\infty} \frac{q_{1}^{r}}{r} \frac{1-q_{2}^{r}}{1-q_{1}^{r}}\left[\frac{\left(q_{1}^{m} v^{\prime}\right)^{r}}{\left(q_{1}^{n} v\right)^{r}}-\frac{\left(q_{1}^{n} v\right)^{r}}{\left(q_{1}^{m} v^{\prime}\right)^{r}}\right]\right) \mathbb{I}_{m}\left(v^{\prime}\right) \mathbb{I}_{n}(v) .
$$

A similar computation gives

$$
\mathbb{I}_{m}^{*}\left(w^{\prime}\right) \mathbb{I}_{n}^{*}(w)=q_{2}^{n-m} \exp \left(\sum_{r=1}^{\infty} \frac{\mathfrak{q}^{2 r} q_{1}^{r}}{r} \frac{1-q_{2}^{r}}{1-q_{1}^{r}}\left[\frac{\left(q_{1}^{n} w\right)^{r}}{\left(q_{1}^{m} w^{\prime}\right)^{r}}-\frac{\left(q_{1}^{m} w^{\prime}\right)^{r}}{\left(q_{1}^{n} w\right)^{r}}\right]\right) \mathbb{I}_{n}^{*}(w) \mathbb{I}_{m}^{*}\left(w^{\prime}\right)
$$

By introducing the function

$$
\Upsilon(\alpha \mid x)=\exp \left(\sum_{r=1}^{\infty} \frac{\alpha^{r}\left(1-q_{2}^{r}\right)}{r\left(1-q_{1}^{r}\right)}\left(x^{r}-x^{-r}\right)\right)
$$

we can write the equations (5.12), (5.14) and (5.15) more compactly;

$$
\begin{aligned}
\mathbb{I}_{m}(v) \mathbb{I}_{n}^{*}(w) & =q_{2}^{m-n} \Upsilon\left(q_{1} \mathfrak{q} \mid \frac{v}{w} q_{1}^{m-n}\right) \mathbb{I}_{n}^{*}(w) \mathbb{I}_{m}(v), \\
\mathbb{I}_{n}(v) \mathbb{I}_{m}\left(v^{\prime}\right) & =q_{2}^{-n+m} \Upsilon\left(q_{1} \mid q_{1}^{m-n} \frac{v^{\prime}}{v}\right) \mathbb{I}_{m}\left(v^{\prime}\right) \mathbb{I}_{n}(v), \\
\mathbb{I}_{m}^{*}\left(w^{\prime}\right) \mathbb{I}_{n}^{*}(w) & =q_{2}^{n-m} \Upsilon\left(\mathfrak{q}^{2} q_{1} \mid q_{1}^{n-m} \frac{w}{w^{\prime}}\right) \mathbb{I}_{n}^{*}(w) \mathbb{I}_{m}^{*}\left(w^{\prime}\right) .
\end{aligned}
$$

Now we are ready to calculate the commutation relation between $\mathcal{T}$ operator and the vector intertwiner $\mathbb{I}_{n}(v)$. Consider the composition $\mathcal{T}_{n}^{m}\left(N, q^{-1} u \mid v, w\right) \mathbb{I}_{k}(z)$ corresponding to figure 4 below.

Then, by using (5.17) and (5.18), we obtain

$$
\begin{aligned}
\mathcal{T}_{n}^{m}\left(N, q_{2}^{-1} u \mid v, w\right) \mathbb{I}_{k}(z) & =\mathbb{I}_{n}^{*}(w) \mathbb{I}_{m}(v) \mathbb{I}_{k}(z)=\mathbb{I}_{n}^{*}(w)\left(q_{2}^{-m+k} \Upsilon\left(q_{1} \mid q_{1}^{k-m} \frac{z}{v}\right)\right) \mathbb{I}_{k}(z) \mathbb{I}_{m}(v) \\
& =q_{2}^{-m+k} \Upsilon\left(q_{1} \mid q_{1}^{k-m} \frac{z}{v}\right) q_{2}^{n-k} \Upsilon\left(q_{1} \mathfrak{q} \mid q_{1}^{k-n} \frac{z}{w}\right)^{-1} \mathbb{I}_{k}(z) \mathbb{I}_{n}^{*}(w) \mathbb{I}_{m}(v) \\
& =q_{2}^{n-m} \Upsilon\left(q_{1} \mid q_{1}^{k-m} \frac{z}{v}\right) \Upsilon\left(q_{1} \mathfrak{q} \mid q_{1}^{n-k} \frac{w}{z}\right) \mathbb{I}_{k}(z) \mathcal{T}_{n}^{m}(N, u \mid v, w) .
\end{aligned}
$$




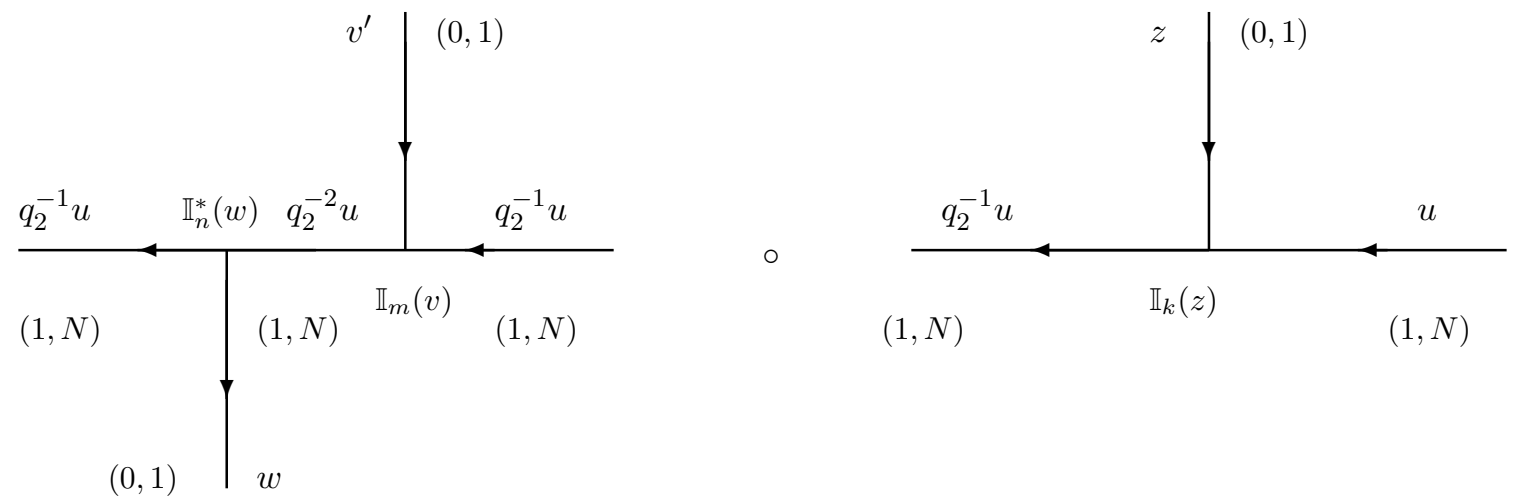

Figure 4. Composition $\circ$ of the vector intertwiner and the shift operator.
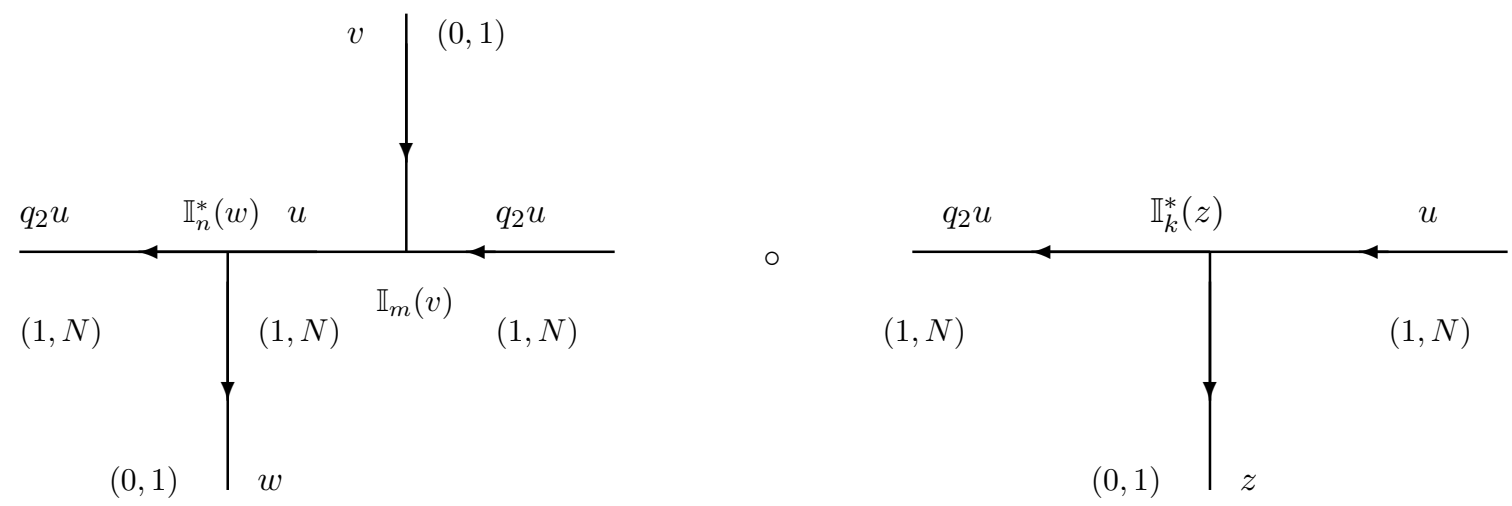

Figure 5. Composition $\circ$ of the dual vector intertwiner and the shift operator.

Here we have used the property $\Upsilon(\alpha \mid x)^{-1}=\Upsilon\left(\alpha \mid \frac{1}{x}\right)$ which follows directly from the definition (5.16). Note that, during the calculation, we do not need to be careful too much about the horizontal spectral parameters, since we have dealt with this issue when we calculated the commutation of the vector intertwiner and dual vector intertwiner. The only thing we need to be careful is the initial point, $\mathcal{T}_{n}^{m}\left(N, q_{2}^{-1} u \mid v, w\right) \mathbb{I}_{k}(z)$, and the final point, $\mathbb{I}_{k}(z) \mathcal{T}_{n}^{m}(N, u \mid v, w)$.

Now consider the composition $\mathcal{T}_{n}^{m}\left(N, q_{2} u \mid v, w\right) \mathbb{I}_{k}^{*}(z)$ corresponding to figure 5 below. We can compute

$$
\begin{aligned}
\mathcal{T}_{n}^{m}\left(N, q_{2} u \mid v, w\right) \mathbb{I}_{k}^{*}(z) & =\mathbb{I}_{n}^{*}(w) \mathbb{I}_{m}(v) \mathbb{I}_{k}^{*}(z)=q_{2}^{m-k} \Upsilon\left(q_{1} \mathfrak{q} \mid \frac{v}{z} q_{1}^{m-k}\right) \mathbb{I}_{n}^{*}(w) \mathbb{I}_{k}^{*}(z) \mathbb{I}_{m}(v) \\
& =q_{2}^{m-k} \Upsilon\left(q_{1} \mathfrak{q} \mid \frac{v}{z} q_{1}^{m-k}\right) q_{2}^{k-n} \Upsilon\left(\mathfrak{q}^{2} q_{1} \mid q_{1}^{k-n} \frac{z}{w}\right) \mathbb{I}_{k}^{*}(z) \mathbb{I}_{n}^{*}(w) \mathbb{I}_{m}(v) \\
& =q_{2}^{m-n} \Upsilon\left(q_{1} \mathfrak{q} \mid \frac{v}{z} q_{1}^{m-k}\right) \Upsilon\left(\mathfrak{q}^{2} q_{1} \mid q_{1}^{k-n} \frac{z}{w}\right) \mathbb{I}_{k}^{*}(z) \mathcal{T}_{n}^{m}(N, u \mid v, w)
\end{aligned}
$$


From eqs. (5.20), (5.21) and the definition (5.7) we see that

$$
\begin{aligned}
& \mathcal{T}_{n}^{+}(N, u \mid v) \mathbb{I}_{k}(z)=\frac{\Upsilon\left(q_{1} \mid q_{1}^{n-k} \mathfrak{q}^{1 / 2} \frac{v}{z}\right)}{\Upsilon\left(q_{1} \mathfrak{q} \mid q_{1}^{n-k} \mathfrak{q}^{-1 / 2} \frac{v}{z}\right)} \mathbb{I}_{k}(z) \mathcal{T}_{n}^{+}\left(N, q_{2} u \mid v\right), \\
& \mathcal{T}_{n}^{-}(N, u \mid v) \mathbb{I}_{k}(z)=\frac{\Upsilon\left(q_{1} \mid q_{1}^{k-n} \mathfrak{q}^{1 / 2} \frac{z}{v}\right)}{\Upsilon\left(q_{1} \mathfrak{q} \mid q_{1}^{k-n} \mathfrak{q}^{-1 / 2} \frac{z}{v}\right)} \mathbb{I}_{k}(z) \mathcal{T}_{n}^{-}\left(N, q_{2} u \mid v\right), \\
& \mathcal{T}_{n}^{+}(N, u \mid v) \mathbb{I}_{k}^{*}(z)=\frac{\Upsilon\left(q_{1} \mathfrak{q} \mid q_{1}^{k-n} \mathfrak{q}^{-1 / 2} \frac{z}{v}\right)}{\Upsilon\left(q_{1} \mathfrak{q}^{2} \mid q_{1}^{k-n} \mathfrak{q}^{1 / 2} \frac{z}{v}\right)} \mathbb{I}_{k}^{*}(z) \mathcal{T}_{n}^{+}\left(N, q_{2}^{-1} u \mid v\right), \\
& \mathcal{T}_{n}^{-}(N, u \mid v) \mathbb{I}_{k}^{*}(z)=\frac{\Upsilon\left(q_{1} \mathfrak{q} \mid q_{1}^{n-k} \mathfrak{q}^{-1 / 2} \frac{v}{z}\right)}{\Upsilon\left(q_{1} \mathfrak{q}^{2} \mid q_{1}^{n-k} \mathfrak{q}^{1 / 2} \frac{v}{z}\right)} \mathbb{I}_{k}^{*}(z) \mathcal{T}_{n}^{-}\left(N, q_{2}^{-1} u \mid v\right) .
\end{aligned}
$$

The function $\Upsilon(\alpha \mid x)$ involves both the positive and the negative powers of the parameter $x$. However the combinations appearing in eqs. (5.22)-(5.25) have only the positive or the negative powers. Hence, we expect that the proportional factors in these equations can be written in terms of the $R$-matrix of the vector representation. We will see this is in fact the case in the next subsection.

\subsection{R-matrix of the vector representation}

We can define the $R$-matrix of the vector representation of the DIM algebra in terms of the universal $R$-matrix $\mathcal{R}_{0}(2.25)$;

$$
\left[\left(\rho_{v_{1}}^{V} \otimes \rho_{v_{2}}^{V}\right)\left(\mathcal{R}_{0}\right)\right]\left[\left[v_{1}\right]_{m} \otimes\left[v_{2}\right]_{l}\right]=R_{m l}\left(\frac{v_{1}}{v_{2}}\right)\left[\left[v_{1}\right]_{m} \otimes\left[v_{2}\right]_{l}\right] .
$$

Thus, to determine the $R_{m l}\left(\frac{v_{1}}{v_{2}}\right)$ we have to calculate the following quantity:

$$
\exp \left(\sum_{r=1}^{\infty} r \kappa_{r} \rho_{v_{1}}^{V}\left(h_{-r}\right) \otimes \rho_{v_{2}}^{V}\left(h_{r}\right)\right)\left[\left[v_{1}\right]_{m} \otimes\left[v_{2}\right]_{l}\right] .
$$

From eqs. (2.3), (3.1) and (3.2), we see that

$$
\rho_{v}^{V}\left(H_{ \pm r}\right)[v]_{i}= \pm \frac{\left(q_{1}^{i} v\right)^{ \pm r}}{r} q_{1}^{(1 \pm 1) r / 2}\left(q_{2}^{ \pm r}-1\right)\left(q_{3}^{ \pm r}-1\right)[v]_{i} .
$$

Hence,

$$
\rho_{v}^{V}\left(h_{ \pm r}\right)[v]_{i}= \pm \frac{1}{\kappa_{r}} \rho_{v}^{V}\left(H_{ \pm r}\right)[v]_{i}=\frac{\left(q_{1}^{i} v\right)^{ \pm r}}{r \kappa_{r}} q_{1}^{(1 \pm 1) r / 2}\left(q_{2}^{ \pm r}-1\right)\left(q_{3}^{ \pm r}-1\right)[v]_{i} .
$$

Accordingly, we obtain

$$
\begin{aligned}
R_{m l}\left(\frac{v_{1}}{v_{2}}\right) & =\exp \left[-\sum_{r=1}^{\infty} \frac{1}{r}\left(q_{1}^{m-l} \frac{v_{1}}{v_{2}}\right)^{-r} \frac{q_{1}^{r}\left(1-q_{2}^{r}\right)\left(1-q_{3}^{r}\right)}{1-q_{1}^{r}}\right] \\
& =\frac{\left(q_{1}^{l-m+1} \frac{v_{2}}{v_{1}} ; q_{1}\right)_{\infty}\left(q_{1}^{l-m} \frac{v_{2}}{v_{1}} ; q_{1}\right)_{\infty}}{\left(\frac{q_{1}^{l-m}}{q_{2}} \frac{v_{2}}{v_{1}} ; q_{1}\right)_{\infty}\left(\frac{q_{1}^{l-m}}{q_{3}} \frac{v_{2}}{v_{1}} ; q_{1}\right)_{\infty}}
\end{aligned}
$$

where we have used the formula (1.12). 
By direct calculation, it is easy to see that the following relation

$$
R_{n l}(x)=\frac{\Upsilon\left(q_{1} \mathfrak{q}^{m} \mid q_{1}^{n-l} \mathfrak{q}^{m} x\right)}{\Upsilon\left(q_{1} \mathfrak{q}^{m+1} \mid q_{1}^{n-l} \mathfrak{q}^{m-1} x\right)}
$$

holds for any $m \in \mathbb{Z}$. Combining (5.31) with $\Upsilon(\alpha \mid x)^{-1}=\Upsilon\left(\alpha \mid x^{-1}\right)$, we can write eqs. (5.22)-(5.25) in terms of the $R$ matrix;

$$
\begin{aligned}
& \mathcal{T}_{n}^{+}(N, u \mid v) \mathbb{I}_{k}(z)=R_{n k}\left(\mathfrak{q}^{1 / 2} \frac{v}{z}\right) \mathbb{I}_{k}(z) \mathcal{T}_{n}^{+}\left(N, q_{2} u \mid v\right), \\
& \mathcal{T}_{n}^{-}(N, u \mid v) \mathbb{I}_{k}(z)=R_{k n}\left(\mathfrak{q}^{1 / 2} \frac{z}{v}\right) \mathbb{I}_{k}(z) \mathcal{T}_{n}^{-}\left(N, q_{2} u \mid v\right), \\
& \mathcal{T}_{n}^{+}(N, u \mid v) \mathbb{I}_{k}^{*}(z)=\frac{1}{R_{n k}\left(\mathfrak{q}^{-1 / 2} \frac{v}{z}\right)} \mathbb{I}_{k}^{*}(z) \mathcal{T}_{n}^{+}\left(N, q_{2}^{-1} u \mid v\right), \\
& \mathcal{T}_{n}^{-}(N, u \mid v) \mathbb{I}_{k}^{*}(z)=\frac{1}{R_{k n}\left(\mathfrak{q}^{-1 / 2} \frac{z}{v}\right)} \mathbb{I}_{k}^{*}(z) \mathcal{T}_{n}^{-}\left(N, q_{2}^{-1} u \mid v\right) .
\end{aligned}
$$

\subsection{KZ equation for the vector intertwiners}

We define the correlation function of vector intertwiners and its duals by

$$
\begin{aligned}
\mathcal{G}_{\lambda_{1}, \cdots, \lambda_{n}}^{\mu_{1}, \cdots, \mu_{m}}\left(u_{n} \mid w_{1}, \cdots, w_{m}, z_{1}, \cdots, z_{n}\right) & =\langle\emptyset| \mathbb{I}_{\mu_{1}}^{*}\left(q_{2}^{-(n-m)} u_{n} \mid w_{1}\right) \cdots \mathbb{I}_{\mu_{m-1}}^{*}\left(q_{2}^{-(n-2)} u_{n} \mid w_{m-1}\right) \mathbb{I}_{\mu_{m}}^{*}\left(q_{2}^{-(n-1)} u_{n} \mid w_{m}\right) \\
& \cdot \mathbb{I}_{\lambda_{1}}\left(q_{2}^{-(n-1)} u_{n} \mid z_{1}\right) \cdots \mathbb{I}_{\lambda_{k}}\left(q_{2}^{-(n-k)} u_{n} \mid z_{k}\right) \cdots \mathbb{I}_{\lambda_{n}}\left(u_{n} \mid z_{n}\right)|\emptyset\rangle .
\end{aligned}
$$

Consider the shift of the spectral parameter of the vector intertwiner;

$$
\begin{aligned}
\left(\mathfrak{q}^{-2}\right)^{z_{k} \frac{\partial}{\partial z_{k}}} & \mathcal{G}_{\lambda_{1}, \cdots, \lambda_{n}}^{\mu_{1}, \cdots, \mu_{m}}\left(u_{n} \mid w_{1}, \cdots, w_{m}, z_{1}, \cdots, z_{n}\right) \\
= & \langle\emptyset| \mathbb{I}_{\mu_{1}}^{*}\left(q_{2}^{-(n-m)} u_{n} \mid w_{1}\right) \cdots \mathbb{I}_{\mu_{m-1}}^{*}\left(q_{2}^{-(n-2)} u_{n} \mid w_{m-1}\right) \mathbb{I}_{\mu_{m}}^{*}\left(q_{2}^{-(n-1)} u_{n} \mid w_{m}\right) \\
& \cdot \mathbb{I}_{\lambda_{1}}\left(q_{2}^{-(n-1)} u_{n} \mid z_{1}\right) \cdots \mathbb{I}_{\lambda_{k}}\left(q_{2}^{-(n-k)} u_{n} \mid \mathfrak{q}^{-2} z_{k}\right) \cdots \mathbb{I}_{\lambda_{n}}\left(u_{n} \mid z_{n}\right)|\emptyset\rangle
\end{aligned}
$$

Here we explicitly write the information of incoming horizontal for each vector intertwiner. The only independent horizontal spectral parameter is of the incoming horizontal of $\mathbb{I}_{\lambda_{n}}$. The other parameters are determined automatically by the property of vector intertwiners. From (5.8) we know that

$$
\begin{aligned}
& \mathbb{I}_{\lambda_{k}}\left(q_{2}^{-(n-k)} u_{n} \mid \mathfrak{q}^{-2} z_{k}\right)=\exp \left(\sum_{r=1}^{\infty} \frac{q_{1}^{r}}{r} \frac{1-q_{2}^{r}}{1-q_{1}^{r}}\left(1-\mathfrak{q}^{2 r}\right)\right) \\
& \cdot \mathcal{T}_{\lambda_{k}}^{-}\left(N, q_{2}^{-(n-k)} u_{n} \mid \mathfrak{q}^{-3 / 2} z_{k}\right) \cdot \mathbb{I}_{\lambda_{k}}\left(q_{2}^{-(n-k)} u_{n} \mid z_{k}\right) \cdot \mathcal{T}_{\lambda_{k}}^{+}\left(N, q_{2}^{-(n-k)} u_{n} \mid \mathfrak{q}^{-1 / 2} z_{k}\right)
\end{aligned}
$$


Hence, we can rewrite $\mathbb{I}_{\lambda_{k}}\left(\mathfrak{q}^{-(n-k)} u_{n} \mid \mathfrak{q}^{-2} z_{k}\right)$ in (5.37) by using (5.38), which implies

$$
\begin{aligned}
&\left(\mathfrak{q}^{-2}\right)^{z_{k} \frac{\partial}{\partial z_{k}}} \mathcal{G}_{\lambda_{1}, \cdots, \lambda_{n}}^{\mu_{1}, \cdots, \mu_{m}}\left(u_{n} \mid w_{1}, \cdots, w_{m}, z_{1}, \cdots, z_{n}\right) \\
&=\left\langle\langle| \mathbb{I}_{\mu_{1}}^{*}\left(q_{2}^{-(n-m)} u_{n} \mid w_{1}\right) \cdots \mathbb{I}_{\mu_{m-1}}^{*}\left(q_{2}^{-(n-2)} u_{n} \mid w_{m-1}\right) \mathbb{I}_{\mu_{m}}^{*}\left(q_{2}^{-(n-1)} u_{n} \mid w_{m}\right)\right. \\
& \cdot \mathbb{I}_{\lambda_{1}}\left(q_{2}^{-(n-1)} u_{n} \mid z_{1}\right) \cdots \mathbb{I}_{\lambda_{k-1}}\left(q_{2}^{-(n-k+1)} u_{n} \mid z_{k-1}\right) \\
& \cdot \mathcal{T}_{\lambda_{k}}^{-}\left(N, q_{2}^{-(n-k+1)} u_{n} \mid \mathfrak{q}^{-3 / 2} z_{k}\right) \cdot \mathbb{I}_{\lambda_{k}}\left(q_{2}^{-(n-k)} u_{n} \mid z_{k}\right) \cdot \mathcal{T}_{\lambda_{k}}^{+}\left(N, q_{2}^{-(n-k)} u_{n} \mid \mathfrak{q}^{-1 / 2} z_{k}\right) \\
& \cdot \mathbb{I}_{\lambda_{k+1}}\left(q_{2}^{-(n-k-1)} u_{n} \mid z_{k+1}\right) \cdots \mathbb{I}_{\lambda_{n}}\left(u_{n} \mid z_{n}\right)|\emptyset\rangle \\
& \cdot \exp \left(\sum_{r=1}^{\infty} \frac{q_{1}^{r}}{r} \frac{1-q_{2}^{r}}{1-q_{1}^{r}}\left(1-\mathfrak{q}^{2 r}\right)\right) .
\end{aligned}
$$

Now by using (5.32), (5.33) and (5.35), we can transport $\mathcal{T}_{\lambda_{k}}^{-}$and $\mathcal{T}_{\lambda_{k}}^{+}$in (5.39) until they hit the left and the right vacua. Then, using (5.5) and (5.6), we calculate $\langle\emptyset| \mathcal{T}_{\lambda_{k}}^{-}\left(N, q_{2}^{m-n} u_{n} \mid \mathfrak{q}^{-3 / 2} z_{k}\right)$ and $\mathcal{T}_{\lambda_{k}}^{+}\left(N, u_{n} \mid \mathfrak{q}^{-1 / 2} z_{k}\right)|\emptyset\rangle$. The result cancels the exponential factor in (5.39). Hence, we finally obtain

$$
\begin{aligned}
& \left(\mathfrak{q}^{-2}\right)^{z_{k} \frac{\partial}{\partial z_{k}}} \mathcal{G}_{\lambda_{1}, \cdots, \lambda_{n}}^{\mu_{1}, \cdots, \mu_{m}}\left(u_{n} \mid w_{1}, \cdots, w_{m}, z_{1}, \cdots, z_{n}\right)=A_{k} \cdot \mathcal{G}_{\lambda_{1}, \cdots, \lambda_{n}}^{\mu_{1}, \cdots, \mu_{m}}\left(u_{n} \mid w_{1}, \cdots, w_{m}, z_{1}, \cdots, z_{n}\right), \\
& A_{k}:=\prod_{j=1}^{m}\left[R_{\mu_{j} \lambda_{k}}\left(\frac{\mathfrak{q} w_{j}}{z_{k}}\right)\right] \prod_{j=1}^{k-1}\left[R_{\lambda_{j} \lambda_{k}}\left(\mathfrak{q}^{2} \frac{z_{j}}{z_{k}}\right)^{-1}\right] \prod_{j=k+1}^{n}\left[R_{\lambda_{k} \lambda_{j}}\left(\frac{z_{k}}{z_{j}}\right)\right] .
\end{aligned}
$$

Now we consider the shift of the spectral parameter of the dual vector intertwiner;

$$
\begin{aligned}
\left(\mathfrak{q}^{-2}\right)^{w_{j} \frac{\partial}{\partial w_{j}}} & \mathcal{G}_{\lambda_{1}, \cdots, \lambda_{n}}^{\mu_{1}, \cdots, \mu_{m}}\left(u_{n} \mid w_{1}, \cdots, w_{m}, z_{1}, \cdots, z_{n}\right) \\
= & \langle\emptyset| \mathbb{I}_{\mu_{1}}^{*}\left(q_{2}^{-(n-m)} u_{n} \mid w_{1}\right) \cdots \mathbb{I}_{\mu_{j-1}}^{*}\left(q_{2}^{-(n-m+j-2)} u_{n} \mid w_{j-1}\right) \\
& \cdot \mathbb{I}_{\mu_{j}}^{*}\left(q_{2}^{-(n-m+j-1)} u_{n} \mid \mathfrak{q}^{-2} w_{j}\right) \mathbb{I}_{\mu_{j+1}}^{*}\left(q_{2}^{-(n-m+j)} u_{n} \mid w_{j+1}\right) \cdots \mathbb{I}_{\mu_{m}}^{*}\left(q_{2}^{-(n-1)} u_{n} \mid w_{m}\right) \\
& \cdot \mathbb{I}_{\lambda_{1}}\left(q_{2}^{-(n-1)} u_{n} \mid z_{1}\right) \cdots \mathbb{I}_{\lambda_{n}}\left(u_{n} \mid z_{n}\right)|\emptyset\rangle .
\end{aligned}
$$

We can rewrite

$$
\begin{aligned}
& \mathbb{I}_{\mu_{j}}^{*}\left(q_{2}^{-(n-m+j-1)} u_{n} \mid \mathfrak{q}^{-2} w_{j}\right)=\exp \left[\sum_{r=1}^{\infty} \frac{q_{1}^{r}\left(1-q_{2}^{r}\right)}{r\left(1-q_{1}^{r}\right)}\left(\mathfrak{q}^{2 r}-1\right)\right] \\
& \cdot \mathcal{T}_{\mu_{j}}^{-}\left(N, q_{2}^{-(n-m+j-1)} u_{n} \mid \mathfrak{q}^{-1 / 2} w_{j}\right)^{-1} \cdot \mathbb{I}_{\mu_{j}}^{*}\left(w_{j}\right) \cdot \mathcal{T}_{\mu_{j}}^{+}\left(N, q_{2}^{-(n-m+j)} u_{n} \mid \mathfrak{q}^{-3 / 2} w_{j}\right)^{-1} .
\end{aligned}
$$

Now we move the operator $\mathcal{T}_{\mu_{j}}^{+}$to the rightmost and $\mathcal{T}_{\mu_{j}}^{-}$to the leftmost as above. By using (5.5) and (5.6), we can calculate $\langle\emptyset| \mathcal{T}_{\mu_{j}}^{-}\left(N, q_{2}^{-(n-m)} u_{n} \mid \mathfrak{q}^{-1 / 2} w_{j}\right)^{-1}$ and $\mathcal{T}_{\mu_{j}}^{+}\left(N, u_{n} \mid \mathfrak{q}^{-3 / 2} w_{j}\right)^{-1}|\emptyset\rangle$. The result of this is the cancellation of the exponential factor in (5.42). The final result is

$$
\begin{aligned}
& \left(\mathfrak{q}^{-2}\right)^{w_{j} \frac{\partial}{\partial w_{j}}} \mathcal{G}_{\lambda_{1}, \cdots, \lambda_{n}}^{\mu_{1}, \cdots, \mu_{m}}\left(u_{n} \mid w_{1}, \cdots, w_{m}, z_{1}, \cdots, z_{n}\right)=A_{j}^{*} \cdot \mathcal{G}_{\lambda_{1}, \cdots, \lambda_{n}}^{\mu_{1}, \cdots, \mu_{m}}\left(u_{n} \mid w_{1}, \cdots, w_{m}, z_{1}, \cdots, z_{n}\right), \\
& A_{j}^{*}:=\prod_{l=1}^{j-1}\left[R_{\mu_{l} \mu_{j}}\left(\frac{w_{l}}{w_{j}}\right)^{-1}\right] \prod_{l=j+1}^{m}\left[R_{\mu_{j} \mu_{l}}\left(\mathfrak{q}^{-2} \frac{w_{j}}{w_{l}}\right)\right] \prod_{k=1}^{n}\left[R_{\mu_{j} \lambda_{k}}\left(\mathfrak{q}^{-1} \frac{w_{j}}{z_{k}}\right)^{-1}\right] .
\end{aligned}
$$

We call the system of eqs. (5.40) and (5.43) generalized KZ equation for the vector representation. 


\subsection{Shift operator from the universal $R$-matrix}

Before proceeding to the generalized KZ equation for the MacMahon representation, in this section we provide an alternative method to obtain the shift operator. This method is based on the use of the universal $R$-matrix of DIM algebra $\mathcal{R}_{0}(2.25)$. Consider the quantity $\left\{\left[\rho_{v}^{V} \otimes \rho^{H}\right]\left(\mathcal{R}_{0}\right)\right\}[v]_{n-1} \otimes|\bullet\rangle$. Here $\rho_{v}^{V}$ is the vector representation with the spectral parameter $v$ described in section 3.1, and $\rho^{H}$ is the horizontal representation described in section 3.5. Up to a proportional factor, we see that

$$
\left\{\left[\rho_{v}^{V} \otimes \rho^{H}\right]\left(\mathcal{R}_{0}\right)\right\}[v]_{n-1} \otimes|\bullet\rangle \sim\left\{\exp \left[\sum_{r=1}^{\infty} r \kappa_{r} \rho^{V}\left(h_{-r}\right) \otimes \rho^{H}\left(h_{r}\right)\right]\right\}\left([v]_{n-1} \otimes|\bullet\rangle\right) .
$$

Eq. (5.29) tells us that

$$
\begin{aligned}
&\left\{\left[\rho_{v}^{V} \otimes \rho^{H}\right]\left(\mathcal{R}_{0}\right)\right\}[v]_{n-1} \otimes|\bullet\rangle \\
& \sim\left\{\exp \left[\sum_{r=1}^{\infty} \frac{q_{1}^{-n r}\left(1-q_{3}^{-r}\right)\left(1-q_{2}^{-r}\right)}{v^{r}} \otimes \frac{\rho^{H}\left(H_{r}\right)}{\kappa_{r}}\right]\right\}\left([v]_{n-1} \otimes|\bullet\rangle\right) \\
&=\left\{\exp \left[-\sum_{r=1}^{\infty} \frac{q_{1}^{-(n-1) r}}{v^{r}} \frac{H_{r}}{1-q_{1}^{r}}\right]\right\}\left([v]_{n-1} \otimes|\bullet\rangle\right) .
\end{aligned}
$$

Note that here we suppress the tensor product notation and $\rho^{H}$. The context will tell us either $H_{r}$ plays a role of generator of the DIM algebra or the operator on a representation. If we compare this operator with eq. (5.6), we see

$$
\left[\rho_{v}^{V} \otimes \rho^{H}\right]\left(\mathcal{R}_{0}\right) \sim \mathcal{T}_{n}^{n}\left(N, u \mid \mathfrak{q}^{1 / 2} v, \mathfrak{q}^{-1 / 2} v\right)^{-1}=\mathcal{T}_{n}^{+}(N, u \mid v) .
$$

Now consider the quantity $\left\{\left[\rho_{v}^{V} \otimes \rho^{H}\right]\left(P \mathcal{R}_{0}\right)\right\}$. Here the operator $P$ switches the position between the elements in tensor product, i.e. $P\left(h_{-r} \otimes h_{r}\right)=h_{r} \otimes h_{-r}$. This time eq. (5.29) implies that

$$
\begin{aligned}
\left\{\exp \left[\sum_{r=1}^{\infty} r \kappa_{r} \rho^{V}\left(h_{r}\right) \otimes \rho^{H}\left(h_{-r}\right)\right]\right\}\left([v]_{n-1} \otimes|\bullet\rangle\right) \\
=\left\{\exp \left[\sum_{r=1}^{\infty}\left(q_{1}^{n} v\right)^{r} \frac{1}{1-q_{1}^{r}} H_{-r}\right]\right\}\left([v]_{n-1} \otimes|\bullet\rangle\right) .
\end{aligned}
$$

If we compare this operator with eq. (5.5), we see that

$$
\left[\rho_{v}^{V} \otimes \rho^{H}\right]\left(P \mathcal{R}_{0}\right) \sim \mathcal{T}_{n}^{n}\left(N, u \mid \mathfrak{q}^{-1 / 2} v, \mathfrak{q}^{1 / 2} v\right)=\mathcal{T}_{n}^{-}(N, u \mid v)
$$

\section{Generalized $\mathrm{KZ}$ equation for MacMahon intertwiners}

\subsection{MacMahon shift operator}

In the last section we learn that the formulas (5.46) and (5.48) provide us the shift operators $\mathcal{T}^{ \pm}$from the universal $R$ matrix. Replacing the vector representation with the MacMahon 
representation, we thus consider the quantity

$$
\begin{aligned}
& \left\{\left[\rho_{v, K_{1}}^{M} \otimes \rho^{H}\right]\left(\mathcal{R}_{0}\right)\right\}\left(\left|\Pi, v, K_{1}\right\rangle \otimes|\bullet\rangle\right) \\
& \quad=\left\{\left(K_{0}^{-} \otimes \mathfrak{q}^{d_{1}}\right)\left(\mathfrak{q}^{d_{1}} \otimes K_{0}^{-}\right) \exp \left[\sum_{n=1}^{\infty} n \kappa_{n} \rho_{v, K_{1}}^{M}\left(h_{-n}\right) \otimes \rho^{H}\left(h_{n}\right)\right]\right\}\left(\left|\Pi, v, K_{1}\right\rangle \otimes|\bullet\rangle\right),
\end{aligned}
$$

where $\rho_{v, K_{1}}^{M}$ represents the vertical MacMahon module with the spectral parameter $v$ and level $K_{1}$, and $H$ denotes the horizontal Fock module with the spectral parameter $u$. To calculate $\left[\rho_{v, K_{1}}^{M}\left(h_{-n}\right) \otimes \rho^{H}\left(h_{n}\right)\right]\left(\left|\Pi, v, K_{1}\right\rangle \otimes|\bullet\rangle\right)$ we need to know $\left[\rho_{v, K_{1}}^{M}\left(h_{-n}\right)\right]\left(\left|\Pi, v, K_{1}\right\rangle\right)$, which has been calculated in [11]. We systematically state the result as the following lemma; ${ }^{11}$

Lemma 6.1. For any $n(\neq 0) \in \mathbb{Z}$

$$
\rho_{v, K}^{M}\left(h_{n}\right)|\Pi, v, K\rangle=\frac{v^{n}}{n}\left[\frac{1-K^{n}}{\kappa_{n}}+\sum_{(i, j, k) \in \Pi} q_{1}^{n i} q_{2}^{n j} q_{3}^{n k}\right]|\Pi, v, K\rangle .
$$

Thus, we obtain

$$
\begin{aligned}
& \left\{\left[\rho_{v, K_{1}}^{M} \otimes \rho^{H}\right]\left(\mathcal{R}_{0}\right)\right\}\left(\left|\Pi, v, K_{1}\right\rangle \otimes|\bullet\rangle\right) \\
& =\alpha\left\{\exp \left[-\sum_{n=1}^{\infty} n \kappa_{n}\left(\frac{v^{-n}}{n}\left[\frac{1-K^{-n}}{\kappa_{-n}}+\sum_{(i, j, k) \in \Pi} q_{1}^{-n i} q_{2}^{-n j} q_{3}^{-n k}\right] \otimes \frac{H_{n}}{\kappa_{n}}\right)\right]\right\}\left(\left|\Pi, v, K_{1}\right\rangle \otimes|\bullet\rangle\right) .
\end{aligned}
$$

where we have used the fact that $d_{1}$ commutes with $H_{r}$ and $\alpha$ is the multiplicative constant in front of the action of $\left(K_{0}^{-} \otimes \mathfrak{q}^{d_{1}}\right)\left(\mathfrak{q}^{d_{1}} \otimes K_{0}^{-}\right)$on the state $\left|\Pi, v, K_{1}\right\rangle \otimes|\bullet\rangle$. Thus, ${ }^{12}$

$$
\mathcal{T}_{\Pi}^{+}(v, K) \sim \exp \left(\sum_{n=1}^{\infty}\left[\frac{1-K^{-n}}{\kappa_{n}}-\sum_{(i, j, k) \in \Pi} q_{1}^{-n i} q_{2}^{-n j} q_{3}^{-n k}\right] v^{-n} H_{n}\right),
$$

where we have suppressed the tensor product.

Similarly,

$$
\begin{aligned}
&\left\{\left[\rho_{v, K_{1}}^{M} \otimes \rho^{H}\right]\left(\mathcal{P} \mathcal{R}_{0}\right)\right\}\left(\left|\Pi, v, K_{1}\right\rangle \otimes|\bullet\rangle\right) \\
&=\left\{( K _ { 0 } ^ { - } \otimes \mathfrak { q } ^ { d _ { 1 } } ) ( \mathfrak { q } ^ { d _ { 1 } } \otimes K _ { 0 } ^ { - } ) \operatorname { e x p } \left[\sum _ { n = 1 } ^ { \infty } \left(n \kappa_{n} \frac{v^{n}}{n}\left[\frac{1-K^{n}}{\kappa_{n}}+\sum_{(i, j, k) \in \Pi} q_{1}^{n i} q_{2}^{n j} q_{3}^{n k}\right]\right.\right.\right. \\
&\left.\left.\left.\otimes(-1) \frac{H_{-n}}{\kappa_{n}}\right)\right]\right\}\left(\left|\Pi, v, K_{1}\right\rangle \otimes|\bullet\rangle\right) .
\end{aligned}
$$

\footnotetext{
${ }^{11}$ Recall the exchange of $q_{1}$ and $q_{2}$ in the formulas of [11]. We have corrected typos in [11].

${ }^{12}$ The notation $\mathcal{T}_{\Pi}^{+}(N \mid v, K)$ resembles the notation of $\mathcal{T}$ operator of the vector module. However, it is just a matter of notation.
} 
Note that we have applied Lemma 6.1 as before. Thus,

$$
\begin{aligned}
& \left\{\left[\rho_{v, K_{1}}^{M} \otimes \rho^{H}\right]\left(\mathcal{P} \mathcal{R}_{0}\right)\right\}\left(\left|\Pi, v, K_{1}\right\rangle \otimes|\bullet\rangle\right) \\
& =\alpha\left\{\exp \left[-\sum_{n=1}^{\infty}\left(n \kappa_{n} \frac{v^{n}}{n}\left[\frac{1-K^{n}}{\kappa_{n}}+\sum_{(i, j, k) \in \Pi} q_{1}^{n i} q_{2}^{n j} q_{3}^{n k}\right] \otimes \frac{H_{-n}}{\kappa_{n}}\right)\right]\right\}\left(\left|\Pi, v, K_{1}\right\rangle \otimes|\bullet\rangle\right) .
\end{aligned}
$$

Suppressing the tensor product, we are able to conclude that

$$
\mathcal{T}_{\Pi}^{-}(v, K) \sim \exp \left(-\sum_{n=1}^{\infty}\left[\frac{1-K^{n}}{\kappa_{n}}+\sum_{(i, j, k) \in \Pi} q_{1}^{n i} q_{2}^{n j} q_{3}^{n k}\right] v^{n} H_{-n}\right) .
$$

\subsection{Effect of the shift operator}

As in the case of the vector and the Fock intertwiners we expect that we are able to write the shift of the spectral parameter in term of the shift operators $\mathcal{T}^{+}$and $\mathcal{T}^{-}$. Schematically,

$$
\Xi(p v)=\mathcal{T}^{-} \cdot \Xi(v) \cdot \mathcal{T}^{+}
$$

However, the value of $p$ in (6.7) is not arbitrary but only certain appropriate values are allowed. We first determine these appropriate values of $p$.

Recall that the MacMahon intertwiner is given by (see (4.18))

$$
\Xi_{\Lambda}(K ; v)=z_{\Lambda}(K ; v) \mathcal{M}^{[n]}(K) \tilde{\Phi}_{\Lambda}^{[n]}(v) \Gamma_{n}(K ; v)
$$

where $\Gamma_{n}(K ; v)$ and $z_{\Lambda}(K ; v)$ are given by (4.19) and (4.20). Note also that (see (4.21))

$$
\tilde{\Phi}_{\Lambda}^{[n]}(v)=\tilde{\Phi}_{\Lambda^{(1)}}(v) \circ \cdots \circ \tilde{\Phi}_{\Lambda^{(n)}}\left(q_{3}^{n-1} v\right),
$$

where the tilde means the intertwiner without the zero mode factor. In the following, introducing the horizontal spectral parameter $u$, we use the zero mode factor

$$
\mathbf{e}(z)=K^{1 / 2} \frac{\theta_{q_{3}}\left(z^{-1}\right)}{\theta_{q_{3}}\left(K z^{-1}\right)} u .
$$

so that we can write

$$
z_{\Lambda}(K ; v)=\prod_{k=1}^{h(\Lambda)} \prod_{(i, j) \in \Lambda^{(k)}} \frac{K}{\mathfrak{q}^{k-1}} \frac{\theta_{q_{3}}\left(q_{3}^{k-1} / x_{i j k}\right)}{\theta_{q_{3}}\left(K / x_{i j k}\right)} \frac{\theta_{q_{3}}\left(1 /\left(x_{i j k} v\right)\right)}{\theta_{q_{3}}\left(K /\left(x_{i j k} v\right)\right)} u .
$$

Let us calculate $\Xi_{\Lambda}(K ; p v)$. Firstly, we see that

$$
\begin{aligned}
\Gamma_{n}(K ; p v)= & \exp \left(\sum_{r=1}^{\infty} \frac{H_{-r}}{\mathfrak{q}^{r}-\mathfrak{q}^{-r}} \frac{q_{3}^{n r}-K^{r}}{\kappa_{r}}\left(\mathfrak{q}^{-1 / 2} v\right)^{r}\left(p^{r}-1\right)\right) \\
& \cdot \Gamma_{n}(K ; v) \exp \left(\sum_{r=1}^{\infty} \frac{H_{r}}{\mathfrak{q}^{r}-\mathfrak{q}^{-r}} \frac{q_{3}^{-n r}-K^{-r}}{\kappa_{r}}\left(\mathfrak{q}^{1 / 2} v\right)^{-r}\left(p^{-r}-1\right)\right),
\end{aligned}
$$


and

$$
\begin{aligned}
z_{\Lambda}(K ; p v) & =\prod_{k=1}^{h(\Lambda)} \prod_{(i, j) \in \Lambda^{(k)}} \frac{K}{\mathfrak{q}^{k-1}} \frac{\theta_{q_{3}}\left(q_{3}^{k-1} / x_{i j k}\right)}{\theta_{q_{3}}\left(K / x_{i j k}\right)} \frac{\theta_{q_{3}}\left(1 /\left(x_{i j k} p v\right)\right)}{\theta_{q_{3}}\left(K /\left(x_{i j k} p v\right)\right)} u \\
& =z_{\Lambda}(K ; v) \prod_{k=1}^{h(\Lambda)} \prod_{(i, j) \in \Lambda^{(k)}} \frac{\theta_{q_{3}}\left(1 /\left(x_{i j k} p v\right)\right)}{\theta_{q_{3}}\left(K /\left(x_{i j k} p v\right)\right)} \frac{\theta_{q_{3}}\left(K /\left(x_{i j k} v\right)\right)}{\theta_{q_{3}}\left(1 /\left(x_{i j k} v\right)\right)}
\end{aligned}
$$

To calculate $\tilde{\Phi}_{\Lambda}^{[n]}(p v)$ we need to know an explicit expression of $\tilde{\Phi}_{\Lambda}^{[n]}(v)$ first. It is convenient to introduce a notation

$$
\chi_{ \pm r}^{(k)}\left(q_{1}, q_{2}\right):=\sum_{(i, j) \in \Lambda^{(k)}} x_{i j}^{ \pm r}-\frac{1}{\left(1-q_{1}^{ \pm r}\right)\left(1-q_{2}^{ \pm r}\right)},
$$

Using this notation, we state its expression systematically as the following lemma;

\section{Lemma 6.2.}

$$
\begin{aligned}
\tilde{\Phi}_{\Lambda}^{[n]}(v)= & c(\Lambda) \exp \left[\sum_{r=1}^{\infty} \frac{H_{-r}}{\mathfrak{q}^{r}-\mathfrak{q}^{-r}} \sum_{k=1}^{n}\left(\mathfrak{q}^{-1 / 2} q_{3}^{k-1} v\right)^{r} \chi_{r}^{(k)}\left(q_{1}, q_{2}\right)\right] \\
& \cdot \exp \left[-\sum_{r=1}^{\infty} \frac{H_{r}}{\mathfrak{q}^{r}-\mathfrak{q}^{-r}} \sum_{k=1}^{n}\left(\mathfrak{q}^{1 / 2} q_{3}^{k-1} v\right)^{-r} \chi_{-r}^{(k)}\left(q_{1}, q_{2}\right)\right],
\end{aligned}
$$

where $c(\Lambda)$ is a constant which may depend on $\Lambda$.

Proof. By definition,

$$
\begin{aligned}
\tilde{\Phi}_{\Lambda}^{[n]}(v) & =\tilde{\Phi}_{\Lambda^{(1)}}(v) \circ \cdots \circ \tilde{\Phi}_{\Lambda^{(n)}}\left(q_{3}^{n-1} v\right) \\
& =\mathcal{G}_{\Lambda^{(1)}}^{-1} \mathcal{G}_{\Lambda^{(2)}}^{-1} \cdots \mathcal{G}_{\Lambda^{(n)}}^{-1}(\text { exponential factor })
\end{aligned}
$$

where (exponential factor) in the above equation is equal to

$$
\begin{aligned}
& \prod_{k=1}^{n} {\left[\exp \left[\sum_{r=1}^{\infty} \frac{H_{-r}}{\mathfrak{q}^{r}-\mathfrak{q}^{-r}}\left(\mathfrak{q}^{-1 / 2} q_{3}^{k-1} v\right)^{r}\left(\sum_{(i, j) \in \Lambda^{(k)}} x_{i j}^{r}-\frac{1}{\left(1-q_{1}^{r}\right)\left(1-q_{2}^{r}\right)}\right)\right]\right.} \\
&\left.\cdot \exp \left[-\sum_{r=1}^{\infty} \frac{H_{r}}{\mathfrak{q}^{r}-\mathfrak{q}^{-r}}\left(\mathfrak{q}^{1 / 2} q_{3}^{k-1} v\right)^{-r}\left(\sum_{(i, j) \in \Lambda^{(k)}} x_{i j}^{-r}-\frac{1}{\left(1-q_{1}^{-r}\right)\left(1-q_{2}^{-r}\right)}\right)\right]\right]
\end{aligned}
$$

Applying the formula (1.14) to eq. (6.16), we obtain the desired result.

Remark 6.3. Recall that the aim of this subsection is to calculate the appropriate value of $p$ by comparing with the exponential factor appearing in (6.3) and (6.6). For this purpose, only the exponential factor of $H_{ \pm r}$ is instrumental, and we do not pay attention to the form of the proportional factor $c$. For the sake of convenience we introduce the notation $\sim$ to mean equal up to a proportional factor. 
By Lemma 6.2

$$
\begin{aligned}
\tilde{\Phi}_{\Lambda}^{[n]}(p v)= & \exp \left[\sum_{r=1}^{\infty} \frac{\left(p^{r}-1\right) H_{-r}}{\mathfrak{q}^{r}-\mathfrak{q}^{-r}} \sum_{k=1}^{n}\left(\mathfrak{q}^{-1 / 2} q_{3}^{k-1} v\right)^{r} \chi_{r}^{(k)}\left(q_{1}, q_{2}\right)\right] \\
& . \tilde{\Phi}_{\Lambda}^{[n]}(v) \exp \left[-\sum_{r=1}^{\infty} \frac{\left(p^{-r}-1\right) H_{r}}{\mathfrak{q}^{r}-\mathfrak{q}^{-r}} \sum_{k=1}^{n}\left(\mathfrak{q}^{1 / 2} q_{3}^{k-1} v\right)^{-r} \chi_{-r}^{(k)}\left(q_{1}, q_{2}\right)\right] .
\end{aligned}
$$

Merging (6.12) and (6.17), we see that

$$
\Xi_{\Lambda}(K ; p v) \sim \widetilde{\mathcal{T}}_{\Lambda}^{-}(p) \cdot \Xi_{\Lambda}(K ; v) \cdot \widetilde{\mathcal{T}}_{\Lambda}^{+}(p),
$$

where $\widetilde{\mathcal{T}}_{\Lambda}^{-}(p)$ and $\widetilde{\mathcal{T}}_{\Lambda}^{+}(p)$ are

$$
\exp \left(\sum_{r=1}^{\infty} \frac{\left(p^{r}-1\right) H_{-r}}{\left(\mathfrak{q}^{r}-\mathfrak{q}^{-r}\right)}\left(\mathfrak{q}^{-1 / 2} v\right)^{r}\left\{\frac{q_{3}^{n r}-K^{r}}{\kappa_{r}}+\sum_{k=1}^{n} q_{3}^{r(k-1)} \chi_{r}^{(k)}\left(q_{1}, q_{2}\right)\right\}\right)
$$

and

$$
\exp \left(\sum_{r=1}^{\infty} \frac{\left(p^{-r}-1\right) H_{r}}{\left(\mathfrak{q}^{r}-\mathfrak{q}^{-r}\right)}\left(\mathfrak{q}^{1 / 2} v\right)^{-r}\left\{\frac{q_{3}^{-n r}-K^{-r}}{\kappa_{r}}-\sum_{k=1}^{n} q_{3}^{-r(k-1)} \chi_{-r}^{(k)}\left(q_{1}, q_{2}\right)\right\}\right)
$$

respectively. Again note that when we switch the $\exp \left(H_{-r}\right)$ factor of $\Gamma_{n}(K ; p v)$ and the $\exp \left(-H_{r}\right)$ factor of $\tilde{\Phi}_{\Lambda}^{[n]}(p v)$ the additional proportional factor appears from the formula (1.14). However, under the sign $\sim$ we are able to pay no attention to it.

Now compare the $\exp \left(H_{-r}\right)$ factor of (6.18) with those of (6.6). We would like to find a value of $p$ which renders them to be equal up to a shift of spectral parameter $v$. That is, we require that

$$
\tilde{\mathcal{T}}_{\Lambda}^{-}(p)=\exp \left(-\sum_{n=1}^{\infty}\left[\frac{1-K^{n}}{\kappa_{n}}+\sum_{(i, j, k) \in \Lambda} q_{1}^{n i} q_{2}^{n j} q_{3}^{n k}\right] \frac{v^{n}}{\lambda^{n}} H_{-n}\right) .
$$

for some constant $\lambda$. We find that the above equality holds if and only if

$$
\begin{aligned}
\frac{p^{r}-1}{\mathfrak{q}^{r}-\mathfrak{q}^{-r}} & \left\{\left(\mathfrak{q}^{-1 / 2} v\right)^{r}\left(\frac{q_{3}^{n r}-K^{r}}{\kappa_{r}}\right)+\sum_{k=1}^{n}\left(\mathfrak{q}^{-1 / 2} q_{3}^{k-1} v\right)^{r} \chi_{r}^{(k)}\left(q_{1}, q_{2}\right)\right\} \\
= & -\frac{v^{r}}{\lambda^{r}}\left[\frac{1-K^{n}}{\kappa_{n}}+\sum_{(i, j, k) \in \Lambda} q_{1}^{n i} q_{2}^{n j} q_{3}^{n k}\right] .
\end{aligned}
$$

Recall that we have exchanged $q_{1}$ and $q_{2}$ in the Fock representation; $x_{i j}:=q_{1}^{i-1} q_{2}^{j-1}$, hence $\sum_{k=1}^{n} q_{3}^{(k-1) r} \sum_{(i, j) \in \Lambda^{(k)}} x_{i j}^{r}=\sum_{(i, j, k) \in \Lambda} x_{i j k}^{r}$. We see that for each $r \in \mathbb{Z}^{\geq 1}$, we should have

$$
p^{r}=\frac{\lambda^{r}-\mathfrak{q}^{3 r / 2}+\mathfrak{q}^{-r / 2}}{\lambda^{r}} .
$$

To make the value of $p$ independent of $r$ we have to choose $\lambda=\mathfrak{q}^{3 / 2}$, and hence we get that $p=\mathfrak{q}^{-2}$. Thus we see that the shift parameter is fixed. Thus, we have confirmed that

$$
\widetilde{\mathcal{T}}_{\Lambda}^{-}\left(\mathfrak{q}^{-2}\right)=\mathcal{T}_{\Lambda}^{-}\left(\mathfrak{q}^{-3 / 2} v, K\right)
$$


We expect that the value $p=\mathfrak{q}^{-2}$ also works for the $\exp \left(H_{r}\right)$ as well. More precisely, when $p=\mathfrak{q}^{-2}$ we should check that there exists $\lambda \in \mathbb{C}$ such that

$$
\widetilde{\mathcal{T}}_{\Lambda}^{+}(p)=\mathcal{T}_{\Lambda}^{+}\left(\frac{v}{\lambda}, K\right)
$$

which means

$$
\begin{array}{r}
\left.\frac{\left(p^{-r}-1\right) H_{r}}{\mathfrak{q}^{r}-\mathfrak{q}^{-r}}\left(\mathfrak{q}^{1 / 2} v\right)^{-r}\left\{\frac{q_{3}^{-n r}-K^{-r}}{\kappa_{r}}-\sum_{k=1}^{n}\left(q_{3}^{k-1}\right)^{-r} \chi_{-r}^{(k)}\left(q_{1}, q_{2}\right)\right\}\right) \\
=\kappa_{r}\left(\frac{v^{-r}}{\lambda^{-r}}\left[\frac{1-K^{-r}}{\kappa_{r}}-\sum_{(i, j, k) \in \Lambda} q_{1}^{-r i} q_{2}^{-r j} q_{3}^{-r k}\right] \frac{H_{n}}{\kappa_{n}}\right) .
\end{array}
$$

By using the summation formula for geometric series, the above equality becomes

$$
\left(p^{-r}-1\right)=\lambda^{r}\left(\mathfrak{q}^{3 r / 2}-\mathfrak{q}^{-r / 2}\right) .
$$

If $p=\mathfrak{q}^{-2}$, we obtain $\lambda=\mathfrak{q}^{1 / 2}$. Hence,

$$
\tilde{\mathcal{T}}_{\Lambda}^{+}\left(\mathfrak{q}^{-2}\right)=\mathcal{T}_{\Lambda}^{+}\left(\mathfrak{q}^{-1 / 2} v, K\right)
$$

is satisfied. In summary we get the relation

$$
\Xi_{\Lambda}\left(K ; \mathfrak{q}^{-2} v\right)=K^{|\Lambda|} \mathcal{T}_{\Lambda}^{-}\left(\mathfrak{q}^{-3 / 2} v, K\right) \cdot \Xi_{\Lambda}(K ; v) \cdot \mathcal{T}_{\Lambda}^{+}\left(\mathfrak{q}^{-1 / 2} v, K\right),
$$

where we fix the proportional factor $K^{|\Lambda|}$ in appendix.

\subsection{Case of the dual intertwiner}

Recall that the dual MacMahon intertwiner is;

$$
\Xi_{\Lambda}^{*}(K ; v)=z_{\Lambda}^{*}(K ; v) \mathcal{M}^{[n] *}(K) \tilde{\Phi}_{\Lambda}^{[n] *}(v) \Gamma_{n}^{*}(K ; v),
$$

where $\Gamma_{n}^{*}(K ; v)$ and $z_{\Lambda}^{*}(K ; v)$ are given by (4.48) and (4.49). We also have (see (4.50));

$$
\tilde{\Phi}_{\Lambda}^{[n] *}(v)=\tilde{\Phi}_{\Lambda^{(1)}}^{*}(v) \circ \cdots \circ \tilde{\Phi}_{\Lambda^{(n)}}^{*}\left(q_{3}^{n-1} v\right) .
$$

We will use the zero mode

$$
\mathbf{f}(z)=\frac{\theta_{q_{3}}\left(\mathfrak{q} K z^{-1}\right)}{\theta_{q_{3}}\left(\mathfrak{q} z^{-1}\right)} u^{-1}
$$

so that we can write

$$
z_{\Lambda}^{*}(K ; v)=\prod_{k=1}^{h(\Lambda)} \prod_{(i, j) \in \Lambda^{(k)}}\left(\frac{K^{1 / 2}}{\mathfrak{q}^{k}} \frac{\theta_{q_{3}}\left(q_{3}^{k} x_{i j k}^{-1}\right)}{\theta_{q_{3}}\left(K x_{i j k}^{-1}\right)}\right)^{-1} \frac{\theta_{q_{3}}\left(\mathfrak{q} K \cdot\left(x_{i j k} v\right)^{-1}\right)}{\theta_{q_{3}}\left(\mathfrak{q} \cdot\left(x_{i j k} v\right)^{-1}\right)} u^{-1} .
$$

In order to calculate $\Xi_{\Lambda}^{*}(K ; p v)$ we need to calculate $z_{\Lambda}^{*}(K ; p v), \tilde{\Phi}_{\Lambda}^{[n] *}(p v)$ and $\Gamma_{n}^{*}(K ; p v)$. From the above equations we see that

$$
\begin{aligned}
\Gamma_{n}^{*}(K ; p v)= & \exp \left(-\sum_{r=1}^{\infty} \frac{H_{-r}}{\mathfrak{q}^{r}-\mathfrak{q}^{-r}} \frac{q_{3}^{n r}-K^{r}}{\kappa_{r}}\left(\mathfrak{q}^{1 / 2} v\right)^{r}\left(p^{r}-1\right)\right) \\
& \cdot \Gamma_{n}^{*}(K ; v) \exp \left(-\sum_{r=1}^{\infty} \frac{H_{r}}{\mathfrak{q}^{r}-\mathfrak{q}^{-r}} \frac{q_{3}^{-n r}-K^{-r}}{\kappa_{r}}\left(\mathfrak{q}^{-1 / 2} v\right)^{-r}\left(p^{-r}-1\right)\right),
\end{aligned}
$$


and

$$
z_{\Lambda}^{*}(K ; p v)=z_{\Lambda}^{*}(K ; v) \prod_{k=1}^{h(\Lambda)} \prod_{(i, j) \in \Lambda^{(k)}}\left[\frac{\theta_{q_{3}}\left(\mathfrak{q} \cdot\left(x_{i j k} v\right)^{-1}\right)}{\theta_{q_{3}}\left(\mathfrak{q} K \cdot\left(x_{i j k} v\right)^{-1}\right)} \frac{\theta_{q_{3}}\left(\mathfrak{q} K \cdot\left(x_{i j k} p v\right)^{-1}\right)}{\theta_{q_{3}}\left(\mathfrak{q} \cdot\left(x_{i j k} p v\right)^{-1}\right)}\right] .
$$

Using the notation (6.14), we can state an explicit expression of $\tilde{\Phi}_{\Lambda}^{[n] *}(v)$ as the following lemma;

\section{Lemma 6.4.}

$$
\begin{aligned}
\tilde{\Phi}_{\Lambda}^{[n] *}(v)= & \tilde{c}(\Lambda) \exp \left[-\sum_{m=1}^{n} \sum_{r=1}^{\infty} \frac{H_{-r}}{\mathfrak{q}^{r}-\mathfrak{q}^{-r}}\left(\mathfrak{q}^{1 / 2} q_{3}^{m-1} v\right)^{r} \chi_{r}^{(m)}\left(q_{1}, q_{2}\right)\right] \\
& \cdot \exp \left[\sum_{m=1}^{n} \sum_{r=1}^{\infty} \frac{H_{r}}{\mathfrak{q}^{r}-\mathfrak{q}^{-r}}\left(\mathfrak{q}^{-1 / 2} q_{3}^{m-1} v\right)^{-r} \chi_{-r}^{(m)}\left(q_{1}, q_{2}\right)\right] .
\end{aligned}
$$

where $\tilde{c}(\Lambda)$ is a constant which may depend on $\Lambda$.

Proof. By definition

$$
\begin{aligned}
\tilde{\Phi}_{\Lambda}^{[n] *}(v) & =\tilde{\Phi}_{\Lambda^{(1)}}^{*}(v) \circ \cdots \circ \tilde{\Phi}_{\Lambda^{(n)}}^{*}\left(q_{3}^{n-1} v\right) \\
& =\tilde{\mathcal{G}}_{\Lambda^{(1)}}^{-1} \cdots \tilde{\mathcal{G}}_{\Lambda^{(n)}}^{-1}(\text { exponential factor })
\end{aligned}
$$

where (exponential factor) is

$$
\begin{aligned}
\prod_{m=1}^{n} & {\left[\exp \left[\sum_{r=1}^{\infty} \frac{H_{-r}}{\mathfrak{q}^{r}-\mathfrak{q}^{-r}}\left(\mathfrak{q}^{1 / 2} q_{3}^{m-1} v\right)^{r}\left(\frac{1}{\left(1-q_{1}^{r}\right)\left(1-q_{2}^{r}\right)}-\sum_{(i, j) \in \Lambda^{(m)}} x_{i j}^{r}\right)\right]\right.} \\
& \left.\cdot \exp \left[\sum_{r=1}^{\infty} \frac{H_{r}}{\mathfrak{q}^{r}-\mathfrak{q}^{-r}}\left(\mathfrak{q}^{-1 / 2} q_{3}^{m-1} v\right)^{-r}\left(\sum_{(i, j) \in \Lambda^{(m)}} x_{i j}^{-r}-\frac{1}{\left(1-q_{1}^{-r}\right)\left(1-q_{2}^{-r}\right)}\right)\right]\right]
\end{aligned}
$$

Applying (1.14) to eq. (6.35), we obtain the desired result.

Now Lemma 6.4 implies that

$$
\begin{aligned}
\tilde{\Phi}_{\Lambda}^{[n] *}(p v)= & \exp \left[-\sum_{m=1}^{n} \sum_{r=1}^{\infty} \frac{\left(p^{r}-1\right) H_{-r}}{\mathfrak{q}^{r}-\mathfrak{q}^{-r}}\left(\mathfrak{q}^{1 / 2} q_{3}^{m-1} v\right)^{r} \chi_{r}^{(m)}\left(q_{1}, q_{2}\right)\right] \\
& . \tilde{\Phi}_{\Lambda}^{[n] *}(v) \cdot \exp \left[\sum_{m=1}^{n} \sum_{r=1}^{\infty} \frac{\left(p^{-r}-1\right) H_{r}}{\mathfrak{q}^{r}-\mathfrak{q}^{-r}}\left(\mathfrak{q}^{-1 / 2} q_{3}^{m-1} v\right)^{-r} \chi_{-r}^{(m)}\left(q_{1}, q_{2}\right)\right] .
\end{aligned}
$$

Now we merge the results (6.32) and (6.36) together. Then, we see that

$$
\begin{aligned}
& \Xi_{\Lambda}^{*}(K ; p v) \\
& \sim \exp \left[-\sum_{r=1}^{\infty} \frac{\left(p^{r}-1\right) H_{-r}}{\mathfrak{q}^{r}-\mathfrak{q}^{-r}}\left(\mathfrak{q}^{1 / 2} v\right)^{r}\left\{\sum_{m=1}^{n} q_{3}^{(m-1) r} \chi_{r}^{(m)}\left(q_{1}, q_{2}\right)+\frac{q_{3}^{n r}-K^{r}}{\kappa_{r}}\right\}\right] \cdot \Xi_{\Lambda}^{*}(K ; v) \\
& \cdot \exp \left[\sum_{r=1}^{\infty} \frac{\left(p^{-r}-1\right) H_{r}}{\mathfrak{q}^{r}-\mathfrak{q}^{-r}}\left(\mathfrak{q}^{-1 / 2} v\right)^{-r}\left\{\sum_{m=1}^{n} q_{3}^{-(m-1) r} \chi_{-r}^{(m)}\left(q_{1}, q_{2}\right)-\frac{q_{3}^{-n r}-K^{-r}}{\kappa_{r}}\right\}\right] .
\end{aligned}
$$

It is straightforward to see that if we take $p=\mathfrak{q}^{-2}$,

$$
\Xi_{\Lambda}^{*}\left(K ; \mathfrak{q}^{-2} v\right)=K^{-|\Lambda|} \mathcal{T}_{\Lambda}^{-}\left(\mathfrak{q}^{-1 / 2} v, K\right)^{-1} \cdot \Xi_{\Lambda}^{*}(K ; v) \cdot \mathcal{T}_{\Lambda}^{+}\left(\mathfrak{q}^{-3 / 2} v, K\right)^{-1},
$$

where we explicitly determine the proportional factor $K^{-|\Lambda|}$ in appendix. 


\subsection{MacMahon KZ equation}

All the commutation relations between the MacMahon intertwiners and the shift operators are obtained by using equation (1.14). It turns out that we can write the commutation relations compactly by introducing

$$
\tilde{R}_{\Pi, \Lambda}^{K_{1}, K_{2}}\left(\frac{u}{v}\right):=\exp \left[-\sum_{r=1}^{\infty} \frac{\kappa_{r}}{r}\left(\frac{u}{v}\right)^{-r}\left(\sum_{(i, j, k) \in \Lambda} x_{i j k}^{r}+\frac{1-K_{2}^{r}}{\kappa_{r}}\right)\left(\sum_{(i, j, k) \in \Pi} x_{i j k}^{-r}-\frac{1-K_{1}^{-r}}{\kappa_{r}}\right)\right],
$$

The commutation relations are

$$
\begin{aligned}
& \mathcal{T}_{\Lambda}^{+}(v, K) \Xi_{\Lambda^{\prime}}\left(K^{\prime}, v^{\prime}\right)=\tilde{R}_{\Lambda, \Lambda^{\prime}}^{K, K^{\prime}}\left(\frac{v}{\mathfrak{q}^{-1 / 2} v^{\prime}}\right) \cdot \Xi_{\Lambda^{\prime}}\left(K^{\prime}, v^{\prime}\right) \mathcal{T}_{\Lambda}^{+}(v, K), \\
& \mathcal{T}_{\Lambda}^{+}(v, K) \Xi_{\Lambda^{\prime}}^{*}\left(K^{\prime}, v^{\prime}\right)=\tilde{R}_{\Lambda, \Lambda^{\prime}}^{K, K^{\prime}}\left(\frac{v}{\mathfrak{q}^{1 / 2} v^{\prime}}\right)^{-1} \cdot \Xi_{\Lambda^{\prime}}^{*}\left(K^{\prime}, v^{\prime}\right) \mathcal{T}_{\Lambda}^{+}(v, K), \\
& \mathcal{T}_{\Lambda}^{-}(v, K) \Xi_{\Lambda^{\prime}}\left(K^{\prime}, v^{\prime}\right)=\tilde{R}_{\Lambda^{\prime}, \Lambda}^{K^{\prime}, K}\left(\frac{v^{\prime}}{\mathfrak{q}^{-1 / 2} v}\right) \cdot \Xi_{\Lambda^{\prime}}\left(K^{\prime}, v^{\prime}\right) \mathcal{T}_{\Lambda}^{-}(v, K), \\
& \mathcal{T}_{\Lambda}^{-}(v, K) \Xi_{\Lambda^{\prime}}^{*}\left(K^{\prime}, v^{\prime}\right)=\tilde{R}_{\Lambda^{\prime}, \Lambda}^{K^{\prime}, K}\left(\frac{v^{\prime}}{\mathfrak{q}^{1 / 2} v}\right)^{-1} \cdot \Xi_{\Lambda^{\prime}}^{*}\left(K^{\prime}, v^{\prime}\right) \mathcal{T}_{\Lambda}^{-}(v, K) .
\end{aligned}
$$

They should be compared with the corresponding relations (5.32)-(5.35) for the intertwiners of the vector representation.

We can determine the relation between $\tilde{R}_{\Pi, \Lambda}^{K_{1}, K_{2}}\left(\frac{u}{v}\right)$ and the MacMahon R-matrix $R_{\Pi, \Lambda}^{K_{1}, K_{2}}\left(\frac{u}{v}\right)$ which appears in the paper [11]. From (6.39) we get that

$$
\tilde{R}_{\Pi, \Lambda}^{K_{1}, K_{2}}\left(\frac{u}{v}\right)=K_{1}^{\frac{-|\Lambda|}{2}} K_{2}^{-\frac{|\Pi|}{2}} R_{\Pi, \Lambda}^{K_{1}, K_{2}}\left(\frac{u}{v}\right) \exp \left[\sum_{r=1}^{\infty} \frac{1}{r}\left(\frac{u}{v}\right)^{-r} \frac{\left(1-K_{2}^{r}\right)\left(1-K_{1}^{-r}\right)}{\kappa_{r}}\right] .
$$

Note that the factor

$$
\exp \left[\sum_{r=1}^{\infty} \frac{1}{r}\left(\frac{u}{v}\right)^{-r} \frac{\left(1-K_{2}^{r}\right)\left(1-K_{1}^{-r}\right)}{\kappa_{r}}\right]
$$

is the vacuum contribution to $\tilde{R}_{\Pi, \Lambda}^{K_{1}, K_{2}}$ or the normalization factor to make $R_{\varnothing, \varnothing}^{K_{1}, K_{2}}=1$.

Now we are ready to derive the generalized KZ equation for MacMahon intertwiner. We define the correlation function by

$$
G_{\Lambda^{1} \cdots \Lambda^{n}}^{\Omega^{1} \ldots \Omega^{m}}\left(\begin{array}{c}
K_{1}^{\prime}, \ldots, K_{m}^{\prime}, K_{1} \ldots, K_{n} \\
w_{1}, \ldots, w_{m}, v_{1}, \ldots, v_{n}
\end{array}\right):=\left\langle\emptyset\left|\Xi_{\Omega^{1}}^{*}\left(K_{1}^{\prime} ; w_{1}\right) \cdots \Xi_{\Omega^{m}}^{*}\left(K_{m}^{\prime} ; w_{m}\right) \Xi_{\Lambda^{1}}\left(K_{1} ; v_{1}\right) \cdots \Xi_{\Lambda^{n}}\left(K_{n} ; v_{n}\right)\right| \emptyset\right\rangle .
$$

Let us first consider the shift of the spectral parameter $v_{i}$;

$$
\begin{aligned}
& \left(\mathfrak{q}^{-2}\right)^{v_{i} \frac{\partial}{\partial v_{i}}} G_{\Lambda^{1 \ldots} \Lambda^{n}}^{\Omega^{1} \ldots \Omega^{m}}\left(\begin{array}{l}
K_{1}^{\prime}, \ldots, K_{m}^{\prime}, K_{1} \ldots, K_{n} \\
w_{1}, \ldots, w_{m}, v_{1}, \ldots, v_{n}
\end{array}\right) \\
& =\langle\emptyset| \Xi_{\Omega^{1}}^{*}\left(K_{1}^{\prime} ; w_{1}\right) \cdots \Xi_{\Omega^{m}}^{*}\left(K_{m}^{\prime} ; w_{m}\right) \Xi_{\Lambda^{1}}\left(K_{1} ; v_{1}\right) \cdots \Xi_{\Lambda^{i-1}}\left(K_{i-1} ; v_{i-1}\right) \Xi_{\Lambda^{i}}\left(K_{i} ; \mathfrak{q}^{-2} v_{i}\right) \\
& \quad \cdot \Xi_{\Lambda^{i+1}}\left(K_{i+1} ; v_{i+1}\right) \cdots \Xi_{\Lambda^{n}}\left(K_{n} ; v_{n}\right)|\emptyset\rangle .
\end{aligned}
$$


From (6.27), the above equation becomes

$$
\begin{aligned}
K_{i}^{\left|\Lambda_{i}\right|}\langle\emptyset| \Xi_{\Omega^{1}}^{*}\left(K_{1}^{\prime} ; w_{1}\right) \cdots \Xi_{\Omega^{m}}^{*}\left(K_{m}^{\prime} ; w_{m}\right) \Xi_{\Lambda^{1}}\left(K_{1} ; v_{1}\right) \cdots \Xi_{\Lambda^{i-1}}\left(K_{i-1} ; v_{i-1}\right) \\
\cdot \mathcal{T}_{\Lambda^{i}}^{-}\left(\mathfrak{q}^{-3 / 2} v_{i}, K_{i}\right) \cdot \Xi_{\Lambda^{i}}\left(K_{i} ; v_{i}\right) \cdot \mathcal{T}_{\Lambda^{i}}^{+}\left(\mathfrak{q}^{-1 / 2} v_{i}, K_{i}\right) \Xi_{\Lambda^{i+1}}\left(K_{i+1} ; v_{i+1}\right) \cdots \Xi_{\Lambda^{n}}\left(K_{n} ; v_{n}\right)|\emptyset\rangle .
\end{aligned}
$$

Next we move the operator $\mathcal{T}_{\Lambda^{i}}^{+}\left(v_{i}, K_{i}\right)^{-1}$ to the right by using the result in the section 6.4 , which implies

$$
\begin{aligned}
& \mathcal{T}_{\Lambda^{i}}^{+}\left(\mathfrak{q}^{-1 / 2} v_{i}, K_{i}\right) \cdot \Xi_{\Lambda^{i+1}}\left(K_{i+1} ; v_{i+1}\right) \cdots \Xi_{\Lambda^{n}}\left(K_{n} ; v_{n}\right)|\emptyset\rangle \\
& \quad=\left(\prod_{l=i+1}^{n} \tilde{R}_{\Lambda^{i}, \Lambda^{l}}^{K_{i}, K_{k}}\left(\frac{v_{i}}{v_{l}}\right)\right) \Xi_{\Lambda^{i+1}}\left(K_{i+1} ; v_{i+1}\right) \cdots \Xi_{\Lambda^{n}}\left(K_{n} ; v_{n}\right)|\emptyset\rangle .
\end{aligned}
$$

Thus, at this step we see that

$$
\begin{gathered}
\left(\mathfrak{q}^{-2}\right)^{v_{i} \frac{\partial}{\partial v_{i}}} G_{\Lambda^{1} \ldots \Lambda^{n}}^{\Omega^{1}}\left(\begin{array}{l}
K_{1}^{\prime}, \ldots, K_{m}^{\prime}, K_{1} \ldots, K_{n} \\
w_{1}, \ldots, w_{m}, v_{1}, \ldots, v_{n}
\end{array}\right)=K_{i}\left|\Lambda_{i}\right|\left(\prod_{l=i+1}^{n} \tilde{R}_{\Lambda^{i}, \Lambda^{l}}^{K_{i}, K_{l}}\left(\frac{v_{i}}{v_{l}}\right)\right) \\
\cdot\langle\emptyset| \Xi_{\Omega^{1}}^{*}\left(K_{1}^{\prime} ; w_{1}\right) \cdots \Xi_{\Omega^{m}}^{*}\left(K_{m}^{\prime} ; w_{m}\right) \Xi_{\Lambda^{1}}\left(K_{1} ; v_{1}\right) \cdots \Xi_{\Lambda^{i-1}}\left(K_{i-1} ; v_{i-1}\right) \\
\cdot \mathcal{T}_{\Lambda^{i}}^{-}\left(\mathfrak{q}^{-3 / 2} v_{i}, K_{i}\right) \Xi_{\Lambda^{i}}\left(K_{i} ; v_{i}\right) \Xi_{\Lambda^{i+1}}\left(K_{i+1} ; v_{i+1}\right) \cdots \Xi_{\Lambda^{n}}\left(K_{n} ; v_{n}\right)|\emptyset\rangle .
\end{gathered}
$$

We then move the operator $\mathcal{T}_{\Lambda^{i}}^{-}\left(\mathfrak{q}^{-3 / 2} v_{i}, K_{i}\right)$ to the left. In the same manner as above, we obtain

$$
\begin{aligned}
& \left(\mathfrak{q}^{-2}\right)^{v_{i} \frac{\partial}{\partial v_{i}}} G_{\Lambda^{1 \ldots} \ldots \Lambda^{n}}^{\Omega^{1} \ldots \Omega^{m}}\left(\begin{array}{c}
K_{1}^{\prime}, \ldots, K_{m}^{\prime}, K_{1} \ldots, K_{n} \\
w_{1}, \ldots, w_{m}, v_{1}, \ldots, v_{n}
\end{array}\right)=\mathcal{A}_{i} \cdot G_{\Lambda^{1} \ldots \Lambda^{n}}^{\Omega^{1} \ldots \Omega^{m}}\left(\begin{array}{l}
K_{1}^{\prime}, \ldots, K_{m}^{\prime}, K_{1} \ldots, K_{n} \\
w_{1}, \ldots, w_{m}, v_{1}, \ldots, v_{n}
\end{array}\right), \\
& \mathcal{A}_{i}:=K_{i}^{\left|\Lambda^{i}\right|}\left(\prod_{l=i+1}^{n} \tilde{R}_{\Lambda^{i}, \Lambda^{l}}^{K_{i}, K_{l}}\left(\frac{v_{i}}{v_{l}}\right)\right)\left(\prod_{k=1}^{i-1} \tilde{R}_{\Lambda^{k}, \Lambda^{i}}^{K_{k}, K_{i}}\left(\mathfrak{q}^{2} \frac{v_{k}}{v_{i}}\right)^{-1}\right)\left(\prod_{s=1}^{m} \tilde{R}_{\Omega^{s}, \Lambda^{i}}^{K^{\prime}, K_{i}}\left(\mathfrak{q} \frac{w_{s}}{v_{i}}\right)\right) .
\end{aligned}
$$

Next let us consider the shift of the spectral parameter $w_{i}$;

$$
\begin{aligned}
& \left(\mathfrak{q}^{-2}\right)^{w_{i} \frac{\partial}{\partial w_{i}}} G_{\Lambda^{1 \ldots} \ldots \Lambda^{n}}^{\Omega^{1} \ldots \Omega^{m}}\left(\begin{array}{c}
K_{1}^{\prime}, \ldots, K_{m}^{\prime}, K_{1} \ldots, K_{n} \\
w_{1}, \ldots, w_{m}, v_{1}, \ldots, v_{n}
\end{array}\right) \\
& =\langle\emptyset| \Xi_{\Omega^{1}}^{*}\left(K_{1}^{\prime} ; w_{1}\right) \cdots \Xi_{\Omega^{i-1}}^{*}\left(K_{i-1}^{\prime} ; w_{i-1}\right) \Xi_{\Omega^{i}}^{*}\left(K_{i}^{\prime} ; \mathfrak{q}^{-2} w_{i}\right) \Xi_{\Omega^{i+1}}^{*}\left(K_{i+1}^{\prime} ; w_{i+1}\right) \cdots \Xi_{\Omega^{m}}^{*}\left(K_{m}^{\prime} ; w_{m}\right) \\
& \quad \cdot \Xi_{\Lambda^{1}}\left(K_{1} ; v_{1}\right) \cdots \Xi_{\Lambda^{n}}\left(K_{n} ; v_{n}\right)|\emptyset\rangle .
\end{aligned}
$$

From (6.38) we get that (6.52) is equal to

$$
\begin{gathered}
\left(K_{i}^{\prime}\right)^{-\left|\Omega^{i}\right|}\langle\emptyset| \Xi_{\Omega^{1}}^{*}\left(K_{1}^{\prime} ; w_{1}\right) \cdots \Xi_{\Omega^{i-1}}^{*}\left(K_{i-1}^{\prime} ; w_{i-1}\right) \mathcal{T}_{\Omega^{i}}^{-}\left(\mathfrak{q}^{-1 / 2} w_{i}, K_{i}^{\prime}\right)^{-1} \cdot \Xi_{\Omega^{i}}^{*}\left(K_{i}^{\prime} ; w_{i}\right) \mathcal{T}_{\Omega^{i}}^{+}\left(\mathfrak{q}^{-3 / 2} w_{i}, K_{i}^{\prime}\right)^{-1} \\
\cdot \Xi_{\Omega^{i+1}}^{*}\left(K_{i+1}^{\prime} ; w_{i+1}\right) \cdots \Xi_{\Omega^{m}}^{*}\left(K_{m}^{\prime} ; w_{m}\right) \Xi_{\Lambda^{1}}\left(K_{1} ; v_{1}\right) \cdots \Xi_{\Lambda^{n}}\left(K_{n} ; v_{n}\right)|\emptyset\rangle
\end{gathered}
$$

As above we can move $\mathcal{T}_{\Omega^{i}}^{+}$to the right and $\mathcal{T}_{\Omega^{i}}^{-}$to the left by using the result in section 6.4. We see that (6.53) is equal to

$$
\begin{aligned}
& \left(K_{i}^{\prime}\right)^{-\left|\Omega^{i}\right|}\left(\prod_{k=i+1}^{m} \tilde{R}_{\Omega^{i}, \Omega^{k}}^{K_{i}^{\prime}, K_{k}^{\prime}}\left(\mathfrak{q}^{-2} \frac{w_{i}}{w_{k}}\right)\right)\left(\prod_{l=1}^{n} \tilde{R}_{\Omega^{i}, \Lambda^{l}}^{K_{i}^{\prime}, K_{l}}\left(\mathfrak{q}^{-1} \frac{w_{i}}{v_{l}}\right)^{-1}\right)\left(\prod_{s=1}^{i-1} \tilde{R}_{\Omega^{s}, \Omega^{i}}^{K_{i}^{\prime}, K_{i}^{\prime}}\left(\frac{w_{s}}{w_{i}}\right)^{-1}\right) \\
& \cdot\left\langle\emptyset\left|\Xi_{\Omega^{1}}^{*}\left(K_{1}^{\prime} ; w_{1}\right) \cdots \Xi_{\Omega^{i-1}}^{*}\left(K_{i-1}^{\prime} ; w_{i-1}\right) \Xi_{\Omega^{i}}^{*}\left(K_{i}^{\prime} ; w_{i}\right) \cdots \Xi_{\Omega^{m}}^{*}\left(K_{m}^{\prime} ; w_{m}\right) \Xi_{\Lambda^{1}}\left(K_{1} ; v_{1}\right) \cdots \Xi_{\Lambda^{n}}\left(K_{n} ; v_{n}\right)\right| \emptyset\right\rangle .
\end{aligned}
$$


Hence, we obtain

$$
\begin{aligned}
& \left(\mathfrak{q}^{-2}\right)^{w_{i} \frac{\partial}{\partial w_{i}}} G_{\Lambda^{1} \ldots \Lambda^{n}}^{\Omega^{1} \ldots \Omega^{m}}\left(\begin{array}{l}
K_{1}^{\prime}, \ldots, K_{m}^{\prime}, K_{1} \ldots, K_{n} \\
w_{1}, \ldots, w_{m}, v_{1}, \ldots, v_{n}
\end{array}\right)=\mathcal{A}_{i}^{*} \cdot G_{\Lambda^{1} \ldots \Lambda^{n}}^{\Omega^{1} \ldots \Omega^{m}}\left(\begin{array}{l}
K_{1}^{\prime}, \ldots, K_{m}^{\prime}, K_{1} \ldots, K_{n} \\
w_{1}, \ldots, w_{m}, v_{1}, \ldots, v_{n}
\end{array}\right), \\
& \mathcal{A}_{i}^{*}:=\left(K_{i}^{\prime}\right)^{-\left|\Omega^{i}\right|}\left(\prod_{k=i+1}^{m} \tilde{R}_{\Omega^{i}, \Omega^{k}}^{K_{i}^{\prime}, K^{\prime}}\left(\mathfrak{q}^{-2} \frac{w_{i}}{w_{k}}\right)\right)\left(\prod_{l=1}^{n} \tilde{R}_{\Omega_{i}^{i}, \Lambda^{l}}^{K^{\prime}, K_{l}}\left(\mathfrak{q}^{-1} \frac{w_{i}}{v_{l}}\right)^{-1}\right)\left(\prod_{s=1}^{i-1} \tilde{R}_{\Omega^{s}, \Omega^{i}}^{K_{s}^{\prime}, K^{\prime}}\left(\frac{w_{s}}{w_{i}}\right)^{-1}\right) .
\end{aligned}
$$

The system of eqs. (6.51) and (6.55) is the generalized KZ equation for MacMahon intertwiners.

\section{Solutions to the generalized $\mathrm{KZ}$ equation}

In this section we discuss solutions to the generalized $\mathrm{KZ}$ equation derived in the last section. Since the generalized KZ equation is a system of linear difference equations for the vertical spectral parameters of the intertwiners, solutions are given up to a multiplicative constant or a $\mathfrak{q}^{2}=q_{3}$ periodic function. In particular we will drop the normalization factors which are independent of the spectral parameters. ${ }^{13}$ Due to the abelian nature of the $R$ matrices (recall they are diagonal), the solutions factorize into the product or more precisely the ration of the fundamental solutions, namely two point functions, which reminds us of the Wick theorem for free field correlators.

\subsection{Correlation function of the vector intertwiners}

Since both the intertwiner and the dual intertwiner keep the level of the horizontal Fock representation, we may assume that it is zero without loss of generality. Under this assumption $z_{n}(v)$ and $z_{n}^{*}(v)$ are independent of the spectral parameter (see (3.37)) and hence for simplicity we will drop these factors in the following formulas; as mentioned above we expect that the general correlation function $\mathcal{G}_{\lambda_{1}, \cdots, \lambda_{n}}^{\mu_{1}, \cdots, \mu_{m}}\left(u_{n} \mid w_{1}, \cdots, w_{m}, z_{1}, \cdots, z_{m}\right)$ can be expressed in terms of the two-point function. Thus, let us begin with the two-point correlator

$$
A_{n m}(v, w):=\left\langle 0\left|\mathbb{I}_{n}(v) \mathbb{I}_{m}(w)\right| 0\right\rangle=\exp \left[\sum_{r=1}^{\infty}\left(\frac{q_{1}^{n} v}{q_{1}^{m} w}\right)^{-r} \frac{q_{1}^{r}}{r} \frac{1-q_{2}^{r}}{1-q_{1}^{r}}\right]
$$

Thus, we can confirm the generalized KZ equation for two-point function;

$$
\frac{A_{n m}\left(\mathfrak{q}^{-2} v, w\right)}{A_{n m}(v, w)}=R_{n m}(v / w)
$$

By using (7.2) one can show that

$$
\mathcal{G}_{\lambda_{1}, \cdots, \lambda_{n}}^{\mu_{1}, \cdots, \mu_{m}}\left(u_{n} \mid w_{1}, \cdots, w_{m}, z_{1}, \cdots, z_{m}\right) \frac{\prod_{\substack{i, j=1 \\ i<j}}^{n} A_{\lambda_{i} \lambda_{j}}\left(\frac{z_{i}}{z_{j}}\right) \prod_{\substack{i, l=1 \\ l<i}}^{m} A_{\mu_{l} \mu_{i}}\left(\frac{w_{l}}{\mathfrak{q}^{2} w_{i}}\right)}{\prod_{j=1}^{m} \prod_{l=1}^{n} A_{\mu_{j} \lambda_{l}}\left(\frac{\mathfrak{q} w_{j}}{\mathfrak{q}^{2} z_{l}}\right)}
$$

\footnotetext{
${ }^{13}$ This does not mean that the normalization factor is unimportant. For example, the normalization factor of the Fock intertwiner is closely related to the theory of Macdonald function.
} 
solves the generalized KZ equation. We should emphasize again that the formula is valid up to a multiplicative constant.

The AGT correspondence motivates us to compare $A_{n m}(v, w)$ with the Nekrasov factor (1.2). Under the normalization

$$
\tilde{A}_{n m}(v, w):=\frac{A_{n m}(v, w)}{A_{00}(v, w)}=\exp \left[\sum_{r=1}^{\infty}\left(q_{1}^{(m-n) r}-1\right)\left(\frac{v}{w}\right)^{-r} \frac{q_{1}^{r}}{r} \frac{1-q_{2}^{r}}{1-q_{1}^{r}}\right]
$$

by the relation (1.12), we see

$$
\tilde{A}_{n m}(v, w)=\frac{\left(\frac{w q_{1}}{v} ; q_{1}\right)_{\infty}\left(\frac{w q_{1}^{m-n+1} q_{2}}{v} ; q_{1}\right)_{\infty}}{\left(\frac{w q_{1} q_{2}}{v} ; q_{1}\right)_{\infty}\left(\frac{w q_{1}^{m-n+1}}{v} ; q_{1}\right)_{\infty}}=\left\{\begin{array}{l}
\frac{N_{\emptyset, m-n}\left(w / q_{2}, v\right)}{N_{\emptyset, m-n}(w, v)} ; m \geq n \\
\frac{N_{n-m, \emptyset}\left(q_{2} w, v\right)}{N_{n-m, \emptyset}(w, v)} ; n \geq m
\end{array}\right.
$$

where the non-negative integer in the subscript stands for the Young diagram with a single row. The insertion of the screening operator is required to obtain more general solution. In particular with the contour integral associated with the spectral parameter of the screening operators the correlation function (7.3) provides building block of the partition function of three dimensional quiver gauge theories, or the $K$ theoretic lift of the vortex counting function $[1,3,4,12,13,16,38,44,49,50]$. Quite recently an elliptic lift of the correlation function of the vector intertwiners is discussed in [27] based on the "Higgsed" network calculus and the elliptic DIM algebra [29, 37, 40].

\subsection{Correlation function of the MacMahon intertwiners}

We also expect that the general correlation function $G_{\Lambda^{1} \ldots \Lambda^{n}}^{\Omega^{1} \ldots \Omega^{m}}\left(\begin{array}{c}K_{1}^{\prime}, \ldots, K_{m}^{\prime}, K_{1} \ldots, K_{n} \\ w_{1}, \ldots, w_{m}, v_{1}, \ldots, v_{n}\end{array}\right)$ can be expressed in terms of the two-point correlators. We first provide an explicit expression of $\left\langle\emptyset\left|\Xi_{\Lambda}\left(K_{1} ; v\right) \Xi_{\Lambda^{\prime}}\left(K_{2} ; w\right)\right| \emptyset\right\rangle$. Up to the normalization factor such as $\mathcal{G}_{\Lambda}^{-1}$ which is independent of the spectral parameter, it is

$$
\begin{aligned}
A_{\Lambda \Lambda^{\prime}}^{K_{1} K_{2}}(v, w): & =\left\langle\emptyset\left|\Xi_{\Lambda}\left(K_{1} ; v\right) \Xi_{\Lambda^{\prime}}\left(K_{2} ; w\right)\right| \emptyset\right\rangle \\
& =z_{\Lambda}\left(K_{1} ; v\right) z_{\Lambda^{\prime}}\left(K_{2} ; w\right) E_{\Lambda \Lambda^{\prime}}^{K_{1} K_{2}}(v, w),
\end{aligned}
$$

where

$$
\begin{aligned}
& E_{\Lambda \Lambda^{\prime}}^{K_{1} K_{2}}(v, w) \\
& =\exp \left[-\sum_{r=1}^{\infty}\left(\frac{w}{v}\right)^{r}\left(\sum_{(i, j, k) \in \Lambda^{\prime}} x_{i j k}^{r}+\frac{1-K_{2}^{r}}{\kappa_{r}}\right)\left(\sum_{(i, j, k) \in \Lambda} x_{i j k}^{-r}-\frac{1-K_{1}^{-r}}{\kappa_{r}}\right) \frac{\left(1-q_{1}^{r}\right)\left(1-q_{2}^{r}\right)}{r}\right] .
\end{aligned}
$$


But from (6.39) and $\kappa_{r}=\left(q_{1}^{r}-1\right)\left(q_{2}^{r}-1\right)\left(q_{3}^{r}-1\right)$ we obtain

$$
\begin{aligned}
\tilde{R}_{\Pi, \Lambda}^{K_{1}, K_{2}}\left(\frac{v}{u}\right) & \exp \left(\sum_{r=1}^{\infty} \frac{\left(1-q_{1}^{r}\right)\left(1-q_{2}^{r}\right)}{r}\left(\frac{u}{v}\right)^{r}\left(\sum_{(i, j, k) \in \Lambda} x_{i j k}^{r}+\frac{1-K_{2}^{r}}{\kappa_{r}}\right)\left(\sum_{(i, j, k) \in \Pi} x_{i j k}^{-r}-\frac{1-K_{1}^{-r}}{\kappa_{r}}\right)\right) \\
= & \frac{\exp \left(\sum_{r=1}^{\infty} \frac{\left(1-q_{1}^{r}\right)\left(1-q_{2}^{r}\right)}{r}\left(\frac{q_{3} u}{v}\right)^{r}\left(\sum_{(i, j, k) \in \Lambda} x_{i j k}^{r}+\frac{1-K_{2}^{r}}{\kappa_{r}}\right)\left(\sum_{(i, j, k) \in \Pi} x_{i j k}^{-r}-\frac{1-K_{1}^{-r}}{\kappa_{r}}\right)\right)}{=} \frac{E_{\Pi \Lambda}^{K_{1} K_{2}}\left(\mathfrak{q}^{-2} v, u\right)}{E_{\Pi \Lambda}^{K_{1} K_{2}}(v, u)},
\end{aligned}
$$

which is nothing but the generalized KZ equation for two point function up to a factor coming from the zero modes. Hence, we see that $G_{\Lambda^{1 \cdots} \Lambda^{1}}^{\Omega^{1}}\left(\begin{array}{l}K_{1}^{\prime}, \ldots, K_{m}^{\prime}, K_{1} \ldots, K_{n} \\ w_{1}, \ldots, w_{m}, v_{1}, \ldots, v_{n}\end{array}\right)$ has to be proportional to

$$
\frac{\prod_{\substack{j, l=1 \\ j<l}}^{n} E_{\Lambda^{j}, \Lambda^{l}}^{K_{j}, K_{l}}\left(v_{j}, v_{l}\right) \prod_{\substack{l, j=1 \\ j<l}}^{m} E_{\Omega^{j}, \Omega^{l}}^{K_{j}^{\prime}, K_{l}^{\prime}}\left(w_{j}, \mathfrak{q}^{2} w_{l}\right)}{\prod_{l=1}^{n} \prod_{s=1}^{m} E_{\Omega_{s}^{s}, \Lambda^{l}}^{K_{1}^{\prime}, K_{l}}\left(w_{s}, \mathfrak{q} v_{l}\right)} .
$$

More precisely, we get that up to a multiplicative constant

$$
\begin{aligned}
& G_{\Lambda^{1} \ldots \Lambda^{n}}^{\Omega^{1} \ldots \Omega^{m}}\left(\begin{array}{l}
K_{1}^{\prime}, \ldots, K_{m}^{\prime}, K_{1} \ldots, K_{n} \\
w_{1}, \ldots, w_{m}, v_{1}, \ldots, v_{n}
\end{array}\right) \\
& =\left(\prod_{i=1}^{n} z_{\Lambda^{i}}\left(K_{i} ; v_{i}\right)\right)\left(\prod_{i=1}^{m} z_{\Omega^{i}}^{*}\left(K_{i}^{\prime} ; w_{i}\right)\right) \frac{\prod_{\substack{j, l=1 \\
j<l}}^{n} E_{\Lambda^{j}, \Lambda^{l}}^{K_{j}, K_{l}}\left(v_{j}, v_{l}\right) \prod_{\substack{l, j=1 \\
j<l}}^{m} E_{\Omega^{j}, \Omega^{l}}^{K_{j}^{\prime}, K_{l}^{\prime}}\left(w_{j}, \mathfrak{q}^{2} w_{l}\right)}{\prod_{l=1}^{n} \prod_{s=1}^{m} E_{\Omega_{s}^{s}, \Lambda^{l}}^{K_{l}^{\prime}, K_{l}}\left(w_{s}, \mathfrak{q} v_{l}\right)}, \\
& =\frac{\prod_{l=1}^{n} z_{\Lambda^{l}}\left(K_{l} ; v_{l}\right) z_{\Lambda^{l}}\left(K_{l} ; \mathfrak{q} v_{l}\right) \prod_{s=1}^{m} z_{\Omega^{s}}^{*}\left(K_{s}^{\prime} ; w_{s}\right) z_{\Omega^{s}}\left(K_{s}^{\prime} ; w_{s}\right)}{\prod_{\substack{j, l=1 \\
j<l}}^{n} z_{\Lambda^{j}}\left(K_{j} ; v_{j}\right) z_{\Lambda^{l}}\left(K_{l} ; v_{l}\right) \cdot \prod_{\substack{l, j=1 \\
j<l}}^{m} z_{\Omega^{j}}\left(K_{j}^{\prime} ; w_{j}\right) z_{\Omega^{l}}\left(K_{l}^{\prime} ; \mathfrak{q}^{2} w_{l}\right)} \\
& \cdot \frac{\prod_{\substack{j, l=1 \\
j<l}}^{n} A_{\Lambda^{j}, \Lambda^{l}}^{K_{j}, K_{l}}\left(v_{j}, v_{l}\right) \prod_{\substack{l, j=1 \\
j<l}}^{m} A_{\Omega^{j}, \Omega^{l}}^{K_{j}^{\prime}, K_{l}^{\prime}}\left(w_{j}, \mathfrak{q}^{2} w_{l}\right)}{\prod_{l=1}^{n} \prod_{s=1}^{m} A_{\Omega_{s}^{s}, \Lambda^{l}}^{K_{l}^{\prime}, K_{l}}\left(w_{s}, \mathfrak{q} v_{l}\right)} .
\end{aligned}
$$

In view of the AGT correspondence, it is quite curious to see if the solutions to the MacMahon KZ-equation give some generalization of the Nekrasov function. Let us analyze the two-points correlator $A_{\Lambda \Lambda^{\prime}}^{K_{1} K_{2}}(v, w)$ (see (7.6)). Defining the normalized two-point function by

$$
\tilde{A}_{\Lambda \Lambda^{\prime}}^{K_{1} K_{2}}(v, w):=\frac{A_{\Lambda \Lambda^{\prime}}^{K_{1} K_{2}}(v, w)}{A_{\emptyset \emptyset}^{K_{1} K_{2}}(v, w)},
$$

we see that

$$
\log \tilde{A}_{\Lambda \Lambda^{\prime}}^{K_{1} K_{2}}(v, w)=\log \left(z_{\Lambda}\left(K_{1} ; v\right) z_{\Lambda^{\prime}}\left(K_{2} ; w\right)\right)+(\mathrm{I})+(\mathrm{II})+(\mathrm{III}),
$$


where

$$
\begin{aligned}
(\mathrm{I})=-\sum_{r=1}^{\infty}\left(\frac{w}{v}\right)^{r} \frac{\left(1-q_{1}^{r}\right)\left(1-q_{2}^{r}\right)}{r}\left(\sum_{\left(i^{\prime}, j^{\prime}, k^{\prime}\right) \in \Lambda^{\prime}} x_{i^{\prime} j^{\prime} k^{\prime}}^{r} \sum_{(i, j, k) \in \Lambda} x_{i j k}^{-r}\right) \\
=\sum_{\left(i^{\prime}, j^{\prime}, k^{\prime}\right) \in \Lambda^{\prime}} \sum_{(i, j, k) \in \Lambda}\left[\log \left(1-\frac{w x_{i^{\prime}, j^{\prime}, k^{\prime}}}{v x_{i j k}}\right)-\log \left(1-\frac{w q_{2} x_{i^{\prime}, j^{\prime}, k^{\prime}}}{v x_{i j k}}\right)\right. \\
\left.-\log \left(1-\frac{w q_{1} x_{i^{\prime}, j^{\prime}, k^{\prime}}}{v x_{i j k}}\right)+\log \left(1-\frac{w q_{1} q_{2} x_{i^{\prime}, j^{\prime}, k^{\prime}}}{v x_{i j k}}\right)\right] .
\end{aligned}
$$

Since $\kappa_{r}=\left(q_{1}^{r}-1\right)\left(q_{2}^{r}-1\right)\left(q_{3}^{r}-1\right)$, the second term is

$$
(\mathrm{II})=\sum_{r=1}^{\infty}\left(\frac{w}{v}\right)^{r} \frac{1}{r} \cdot \frac{1-K_{1}^{-r}}{q_{3}^{r}-1} \sum_{(i, j, k) \in \Lambda^{\prime}} x_{i j k}^{r}=\sum_{r=1}^{\infty}\left(\frac{w}{v K_{1}}\right)^{r} \frac{1}{r} \cdot \frac{K_{1}^{r}-1}{q_{3}^{r}-1} \sum_{(i, j, k) \in \Lambda^{\prime}} x_{i j k}^{r} .
$$

Using the identity (1.12), we see that eq. (7.14) becomes

$$
\begin{aligned}
& \sum_{r=1}^{\infty}\left(\frac{w}{v K_{1}}\right)^{r} \frac{1}{r}\left[\frac{K_{1}^{r}}{q_{3}^{r}-1}-\frac{1}{q_{3}^{r}-1}\right] \sum_{(i, j, k) \in \Lambda^{\prime}} x_{i j k}^{r} \\
& \quad=-\sum_{(i, j, k) \in \Lambda^{\prime}} \sum_{r=1}^{\infty} \frac{1}{r\left(1-q_{3}^{r}\right)}\left(\frac{w}{v} x_{i j k}\right)^{r}+\sum_{(i, j, k) \in \Lambda^{\prime}} \sum_{r=1}^{\infty} \frac{1}{r\left(1-q_{3}^{r}\right)}\left(\frac{w}{v K_{1}} x_{i j k}\right)^{r} \\
& \quad=\sum_{(i, j, k) \in \Lambda^{\prime}} \log \left(\frac{w}{v} x_{i j k} ; q_{3}\right)_{\infty}-\sum_{(i, j, k) \in \Lambda^{\prime}} \log \left(\frac{w}{v K_{1}} x_{i j k} ; q_{3}\right)_{\infty} .
\end{aligned}
$$

Similarly the third term becomes

$$
\begin{aligned}
\text { (III) } & =\sum_{r=1}^{\infty}\left(\frac{w}{v}\right)^{r} \frac{1}{r} \frac{1-K_{2}^{r}}{1-q_{3}^{r}} \sum_{(i, j, k) \in \Lambda} x_{i j k}^{-r} \\
& =\sum_{(i, j, k) \in \Lambda} \sum_{r=1}^{\infty} \frac{1}{r\left(1-q_{3}^{r}\right)}\left(\frac{w}{v x_{i j k}}\right)^{r}-\sum_{(i, j, k) \in \Lambda} \sum_{r=1}^{\infty} \frac{1}{r\left(1-q_{3}^{r}\right)}\left(\frac{w K_{2}}{v x_{i j k}}\right)^{r} \\
& =-\sum_{(i, j, k) \in \Lambda} \log \left(\frac{w}{v x_{i j k}} ; q_{3}\right)_{\infty}+\sum_{(i, j, k) \in \Lambda} \log \left(\frac{w K_{2}}{v x_{i j k}} ; q_{3}\right)_{\infty} .
\end{aligned}
$$

Now merging all the three terms, we obtain

$$
\begin{aligned}
\tilde{A}_{\Lambda \Lambda^{\prime}}^{K_{1} K_{2}}(v, w)=z_{\Lambda}\left(K_{1} ; v\right) z_{\Lambda^{\prime}}\left(K_{2} ; w\right) \\
\cdot\left[\prod_{\left(i^{\prime}, j^{\prime}, k^{\prime}\right) \in \Lambda^{\prime}} \prod_{(i, j, k) \in \Lambda} \frac{\left(1-\frac{w x_{i^{\prime} j^{\prime} k^{\prime}}}{v x_{i j k}}\right)\left(1-\frac{w q_{1} q_{2} x_{i^{\prime}, j^{\prime}, k^{\prime}}}{v x_{i j k}}\right)}{\left(1-\frac{w q_{2} x_{i^{\prime}, j^{\prime}, k^{\prime}}}{v x_{i j k}}\right)\left(1-\frac{w q_{1} x_{i^{\prime}, j^{\prime}, k^{\prime}}}{v x_{i j k}}\right)}\right] \cdot\left[\prod_{(i, j, k) \in \Lambda^{\prime}} \frac{\left(\frac{w}{v} x_{i j k} ; q_{3}\right)_{\infty}}{\left(\frac{w}{v K_{1}} x_{i j k} ; q_{3}\right)_{\infty}}\right] \\
\cdot\left[\prod_{(i, j, k) \in \Lambda} \frac{\left(\frac{w K_{2}}{v x_{i j k}} ; q_{3}\right)_{\infty}}{\left(\frac{w}{v x_{i j k}} ; q_{3}\right)_{\infty}}\right] .
\end{aligned}
$$

To show the relation of $\tilde{A}_{\Lambda \Lambda^{\prime}}^{K_{1} K_{2}}(v, w)$ and the Nekrasov function, we show that under a certain appropriate limit the inverse of the normalized two-point correlators 
$\tilde{A}_{\Lambda \Lambda^{\prime}}^{K_{1} K_{2}}(v, w)$ reduces to the Nekrasov function up to multiplicative constant. ${ }^{14}$ Using $x_{i j k}=q_{1}^{i-1} q_{2}^{j-1} q_{3}^{k-1}=q_{1}^{i} q_{2}^{j} q_{3}^{k}$, we see that under the conditions $K_{1}=K_{2}=q_{3}$ and $k=1$,

$$
\begin{aligned}
\tilde{A}_{\lambda \lambda^{\prime}}^{q_{3}, q_{3}}(v, w)^{-1}=z_{\lambda}\left(q_{3} ; v\right)^{-1} z_{\lambda^{\prime}}\left(q_{3} ; w\right)^{-1} \\
\cdot\left[\prod_{\left(i^{\prime}, j^{\prime}\right) \in \lambda^{\prime}} \prod_{(i, j) \in \lambda} \frac{\left(1-\frac{w}{v} q_{2} q_{1}^{i^{\prime}-i} q_{2}^{j^{\prime}-j}\right)\left(1-\frac{w}{v} q_{1} q_{1}^{i^{\prime}-i} q_{2}^{j^{\prime}-j}\right)}{\left(1-\frac{w}{v} q_{1}^{i^{\prime}-i} q_{2}^{j^{\prime}-j}\right)\left(1-\frac{w}{v} q_{1} q_{2} q_{1}^{i^{\prime}-i} q_{2}^{j^{\prime}-j}\right)} \cdot\left[\prod_{\left(i^{\prime}, j^{\prime}\right) \in \lambda^{\prime}}\left(1-\frac{w}{v} q_{1}^{i^{\prime}} q_{2}^{j^{\prime}}\right)\right]\right. \\
\cdot\left[\prod_{(i, j) \in \lambda}\left(1-q_{1} q_{2} \frac{w}{v q_{1}^{i} q_{2}^{j}}\right)\right] .
\end{aligned}
$$

Comparing with the Nekrasov function (1.2), we see that

$$
\tilde{A}_{\lambda \lambda^{\prime}}^{q_{3}, q_{3}}(v, w)^{-1}=z_{\lambda}\left(q_{3} ; v\right)^{-1} z_{\lambda^{\prime}}\left(q_{3} ; w\right)^{-1} \cdot N_{\lambda \lambda^{\prime}}(w, v) .
$$

This justifies that $\tilde{A}_{\Lambda \Lambda^{\prime}}^{K_{1} K_{2}}(v, w)^{-1}$ can be regarded as a generalized Nekrasov function.

It is an interesting challenge to understand the solution (7.17) as some kind of the partition function of supersymmetric gauge theory and/or generalization of Macdonald function $[33,45]$. For example, it is tempting to relate it to six dimensional gauge theory. However the direction $q_{3}$ appears as "preferred" in (7.17) and it seems to suggest the existence of an additional defect in the theory. ${ }^{15}$ Though the MacMahon representation is symmetric in $\left(q_{1}, q_{2}, q_{3}\right)$, the horizontal Fock representation breaks it by the commutation relation with $\mathfrak{q}=q_{3}^{1 / 2}$. The appearance of the infinite product is another issue against an interpretation in terms of the gauge theory.

\section{Acknowledgments}

We would like to thank H. Awata, A. Mironov, A. Morozov and Y. Zenkevich for useful discussions. Our work is supported in part by Grants-in-Aid for Scientific Research (\# 18K03274) (H.K.). The work of P.C. is supported by the MEXT Scholarship.

\section{A Determine the proportional factor}

Here we determine the proportional factor of (6.27) explicitly. From (4.18) we get that

$$
\Xi_{\Lambda}(K ; p v)=\left[\frac{z_{\lambda}(K ; p v)}{z_{\lambda}(K ; v)}\right] z_{\lambda}(K ; v) \mathcal{M}^{[n]}(K) \tilde{\Phi}_{\Lambda}^{[n]}(p v) \Gamma_{n}(K ; p v) .
$$

Now we can write

$$
\tilde{\Phi}_{\Lambda}^{[n]}(p v) \Gamma_{n}(K ; p v)=A_{\Lambda}^{(-)}(v ; p) \tilde{\Phi}_{\Lambda}^{[n]}(v) A_{\Lambda}^{(+)}(v ; p) \cdot B_{n}^{(-)}(v, p) \Gamma_{n}(K ; v) B_{n}^{(+)}(v, p),
$$

\footnotetext{
${ }^{14}$ Note that in the ordinary case, the Nekrasov functions appear in the denominator of the two-point correlators.

${ }^{15}$ If we adopt a suggestion in [47] that the MacMahon representation may be associated with a system of $D 7$ and anti- $D 7$ branes, the defect would be five branes attached to it.
} 
where

$$
A_{\Lambda}^{( \pm)}(v ; p)=\exp \left[\mp \sum_{r=1}^{\infty} \frac{\left(p^{\mp r}-1\right) H_{ \pm r}}{\mathfrak{q}^{r}-\mathfrak{q}^{-r}} \sum_{k=1}^{n} \mathfrak{q}^{-r / 2}\left(q_{3}^{k-1} v\right)^{\mp r} \chi_{\mp r}^{(k)}\left(q_{1}, q_{2}\right)\right],
$$

and

$$
B_{n}^{( \pm)}(v, p)=\exp \left(\sum_{r=1}^{\infty} \frac{\left(p^{\mp r}-1\right) H_{ \pm r}}{\mathfrak{q}^{r}-\mathfrak{q}^{-r}} \frac{q_{3}^{\mp n r}-K^{\mp r}}{\kappa_{r}} \mathfrak{q}^{-r / 2} v^{\mp r}\right)
$$

where we have used the notation (6.14). By using (1.14), we get that

$$
A_{\Lambda}^{(+)}(v ; p) B_{n}^{(-)}(v, p)=\exp (\boldsymbol{\beta}) B_{n}^{(-)}(v, p) A_{\Lambda}^{(+)}(v ; p),
$$

where

$$
\boldsymbol{\beta}=-\sum_{r=1}^{\infty}\left(p^{-r}-1\right)\left(p^{r}-1\right)\left(\frac{q_{3}^{n r}-K^{r}}{\kappa_{r}}\right) \frac{\left(1-q_{1}^{r}\right)\left(1-q_{2}^{r}\right)}{r}\left(\sum_{(i, j, k) \in \Lambda} x_{i j k}^{-r}-\frac{1-q_{3}^{-r n}}{\kappa_{r}}\right) \text {. }
$$

Next we would like to switch between $A_{\Lambda}^{(+)}(v ; p)$ and $\Gamma_{n}(K ; v)$. We know the explicit expression of $\Gamma_{n}(K ; v)$ from eq. (4.19). So by using (1.14) again, we can show that

$$
A_{\Lambda}^{(+)}(v ; p) \Gamma_{n}(K ; v)=\exp (\mathbf{\uparrow}) \Gamma_{n}(K ; v) A_{\Lambda}^{(+)}(v ; p)
$$

with

$$
\mathbf{\phi}=-\sum_{r=1}^{\infty}\left(p^{-r}-1\right)\left(\sum_{(i, j, k) \in \Lambda} x_{i j k}^{-r}-\frac{1-q_{3}^{-r n}}{\kappa_{r}}\right) \frac{q_{3}^{n r}-K^{r}}{\kappa_{r}} \frac{\left(1-q_{1}^{r}\right)\left(1-q_{2}^{r}\right)}{r} .
$$

In the last step we would like to switch between $\tilde{\Phi}_{\Lambda}^{[n]}(v)$ and $B_{n}^{(-)}(v, p)$. Again since we know the explicit expression of $\tilde{\Phi}_{\Lambda}^{[n]}(v)$ from Lemma 6.2, and (1.14) implies

$$
\tilde{\Phi}_{\Lambda}^{[n]}(v) B_{n}^{(-)}(v, p)=\exp (\nabla) B_{n}^{(-)}(v, p) \tilde{\Phi}_{\Lambda}^{[n]}(v)
$$

where

$$
\odot=-\sum_{r=1}^{\infty} \frac{q_{3}^{n r}-K^{r}}{\kappa_{r}}\left(p^{r}-1\right) \frac{\left(1-q_{1}^{r}\right)\left(1-q_{2}^{r}\right)}{r}\left(\sum_{(i, j, k) \in \Lambda} x_{i j k}^{-r}-\frac{1-q_{3}^{-r n}}{\kappa_{r}}\right)
$$

Consequently,

$$
\begin{aligned}
\tilde{\Phi}_{\Lambda}^{[n]}(p v) \Gamma_{n}(K ; p v)= & \exp (\boldsymbol{\beta}) \exp (\boldsymbol{(}) \exp (\varnothing) \\
& \cdot A_{\Lambda}^{(-)}(v ; p) B_{n}^{(-)}(v, p) \cdot \tilde{\Phi}_{\Lambda}^{[n]}(v) \Gamma_{n}(K ; v) \cdot A_{\Lambda}^{(+)}(v ; p) B_{n}^{(+)}(v, p) .
\end{aligned}
$$

From the explicit expressions of $\mathbf{p}, \mathbf{p}$, and $\varnothing$ we get that $\mathbf{p}+\varnothing=0$. In conclusion,

$$
\begin{aligned}
& \Xi_{\Lambda}(K ; p v)=\frac{z_{\Lambda}(K ; p v)}{z_{\Lambda}(K ; v)} \\
& \cdot \exp \left(\sum_{r=1}^{\infty} \frac{\left(p^{r}-1\right) H_{-r}}{\left(\mathfrak{q}^{r}-\mathfrak{q}^{-r}\right)}\left\{\left(\mathfrak{q}^{-1 / 2} v\right)^{r}\left(\frac{q_{3}^{n r}-K^{r}}{\kappa_{r}}\right)+\sum_{k=1}^{n}\left(\mathfrak{q}^{-1 / 2} q_{3}^{k-1} v\right)^{r} \chi_{r}^{(k)}\left(q_{1}, q_{2}\right)\right\}\right) \cdot \Xi_{\Lambda}(K ; v) \\
& \cdot \exp \left(\sum_{r=1}^{\infty} \frac{\left(p^{-r}-1\right) H_{r}}{\left(\mathfrak{q}^{r}-\mathfrak{q}^{-r}\right)}\left\{\left(\mathfrak{q}^{1 / 2} v\right)^{-r} \frac{q_{3}^{-n r}-K^{-r}}{\kappa_{r}}-\sum_{k=1}^{n}\left(\mathfrak{q}^{1 / 2} q_{3}^{k-1} v\right)^{-r} \chi_{-r}^{(k)}\left(q_{1}, q_{2}\right)\right\}\right) .
\end{aligned}
$$


When $p=\mathfrak{q}^{-2}=q_{3}^{-1}$, by (1.13) and (6.13) we can show $\frac{z_{\Lambda}\left(K ; \mathfrak{q}^{-2} v\right)}{z_{\Lambda}(K ; v)}=K^{|\Lambda|}$, and hence we arrive at (6.27).

Next we would like to we determine the proportional factor in (6.38) explicitly From eq. (4.47) we know that

$$
\Xi_{\Lambda}^{*}(K ; p v)=\frac{z_{\Lambda}^{*}(K ; p v)}{z_{\Lambda}^{*}(K ; v)} z_{\Lambda}^{*}(K ; v) \mathcal{M}^{[n] *}(K) \tilde{\Phi}_{\Lambda}^{[n] *}(p v) \Gamma_{n}^{*}(K ; p v) .
$$

We can write

$$
\tilde{\Phi}_{\Lambda}^{[n] *}(p v) \Gamma_{n}^{*}(K ; p v)=C_{\Lambda}^{(-)}(v ; p) \tilde{\Phi}_{\Lambda}^{[n] *}(v) C_{\Lambda}^{(+)}(v ; p) \cdot D_{n}^{(-)}(v ; p) \Gamma_{n}^{*}(K ; v) D_{n}^{(+)}(v ; p),
$$

where

$$
C_{\Lambda}^{( \pm)}(v ; p)=\exp \left[ \pm \sum_{m=1}^{n} \sum_{r=1}^{\infty} \frac{\left(p^{\mp r}-1\right) H_{ \pm r}}{\mathfrak{q}^{r}-\mathfrak{q}^{-r}} \mathfrak{q}^{r / 2}\left(q_{3}^{m-1} v\right)^{\mp r} \chi_{\mp r}^{(m)}\left(q_{1}, q_{2}\right)\right]
$$

and

$$
D_{n}^{( \pm)}(v ; p)=\exp \left(-\sum_{r=1}^{\infty} \frac{\left(p^{\mp r}-1\right) H_{ \pm r}}{\mathfrak{q}^{r}-\mathfrak{q}^{-r}} \frac{q_{3}^{\mp n r}-K^{\mp r}}{\kappa_{r}} \mathfrak{q}^{r / 2} v^{\mp r}\right)
$$

By using (1.14), we get that

$$
C_{\Lambda}^{(+)}(v ; p) D_{n}^{(-)}(v ; p)=\exp (\star) D_{n}^{(-)}(v ; p) C_{\Lambda}^{(+)}(v ; p)
$$

where

$$
\star=-\sum_{r=1}^{\infty}\left(p^{-r}-1\right)\left(p^{r}-1\right) \frac{q_{3}^{n r}-K^{r}}{\kappa_{r}} \frac{q_{3}^{r}\left(q_{1}^{r}-1\right)\left(q_{2}^{r}-1\right)}{r} \cdot\left[\sum_{(i, j, k) \in \Lambda} x_{i j k}^{-r}-\frac{1-q_{3}^{-r n}}{\kappa_{r}}\right] .
$$

Next we would like to switch between $C_{\Lambda}^{(+)}(v ; p)$ and $\Gamma_{n}^{*}(K ; v)$. We know the explicit expression of $\Gamma_{n}^{*}(K ; v)$ from (4.48). So by using (1.14) again, we can show that

$$
C_{\Lambda}^{(+)}(v ; p) \Gamma_{n}^{*}(K ; v)=\exp (\mho) \Gamma_{n}^{*}(K ; v) C_{\Lambda}^{(+)}(v ; p),
$$

where

$$
\mho=-\sum_{r=1}^{\infty}\left(p^{-r}-1\right) \frac{q_{3}^{n r}-K^{r}}{\kappa_{r}} \frac{q_{3}^{r}\left(q_{1}^{r}-1\right)\left(q_{2}^{r}-1\right)}{r}\left(\sum_{(i, j, k) \in \Lambda} x_{i j k}^{-r}-\frac{1-q_{3}^{-n r}}{\kappa_{r}}\right) .
$$

Finally we would like to switch between $\tilde{\Phi}_{\Lambda}^{[n] *}(v)$ and $D_{n}^{(-)}(v ; p)$. Again since we know the explicit expression of $\tilde{\Phi}_{\Lambda}^{[n] *}(v)$ from Lemma 6.4 , we can use (1.14) to show that

$$
\tilde{\Phi}_{\Lambda}^{[n] *}(v) D_{n}^{(-)}(v ; p)=\exp (\natural) D_{n}^{(-)}(v ; p) \tilde{\Phi}_{\Lambda}^{[n] *}(v),
$$

where

$$
\natural=-\sum_{r=1}^{\infty}\left(p^{r}-1\right) \frac{q_{3}^{n r}-K^{r}}{k_{r}} \frac{q_{3}^{r}\left(q_{1}^{r}-1\right)\left(q_{2}^{r}-1\right)}{r}\left(\sum_{(i, j, k) \in \Lambda} x_{i j k}^{-r}-\frac{1-q_{3}^{-r n}}{\kappa_{r}}\right) .
$$


Consequently,

$$
\begin{aligned}
\tilde{\Phi}_{\Lambda}^{[n] *}(p v) \Gamma_{n}^{*}(K ; p v)= & \exp (\star) \exp (\mho) \exp (\nvdash) \\
& \cdot C_{\Lambda}^{(-)}(v ; p) D_{n}^{(-)}(v ; p) \cdot \tilde{\Phi}_{\Lambda}^{[n] *}(v) \Gamma_{n}^{*}(K ; v) \cdot C_{\Lambda}^{(+)}(v ; p) D_{n}^{(+)}(v ; p) .
\end{aligned}
$$

Substituting (A.18) into (A.10), we see that the proportional factor of (6.38) is

$$
\frac{z_{\Lambda}^{*}(K ; p v)}{z_{\Lambda}^{*}(K ; v)} \exp (\star+\mho+\natural) .
$$

However, it is easy to show that $\star+\mho+\downarrow=0$. Furthermore, for $p=\mathfrak{q}^{-2}=q_{3}^{-1}$ (1.13) and (6.33) tell us that $\frac{z_{\Omega}^{*}\left(K^{\prime} ; \mathfrak{q}^{-2} w\right)}{z_{\Omega}^{*}\left(K^{\prime} ; w\right)}=\frac{1}{K^{\prime|\Omega|}}$. Hence, we finally obtain (6.38).

Open Access. This article is distributed under the terms of the Creative Commons Attribution License (CC-BY 4.0), which permits any use, distribution and reproduction in any medium, provided the original author(s) and source are credited.

\section{References}

[1] M. Aganagic, E. Frenkel and A. Okounkov, Quantum q-Langlands correspondence, Trans. Moscow Math. Soc. 79 (2018) 1 [arXiv:1701.03146] [INSPIRE].

[2] H. Awata, B. Feigin and J. Shiraishi, Quantum algebraic approach to refined topological vertex, JHEP 03 (2012) 041 [arXiv: 1112.6074] [INSPIRE].

[3] M. Aganagic, N. Haouzi, C. Kozcaz and S. Shakirov, Gauge/Liouville triality, arXiv: 1309.1687 [INSPIRE].

[4] M. Aganagic, N. Haouzi and S. Shakirov, $A_{n}$-triality, arXiv:1403.3657 [INSPIRE].

[5] L.F. Alday, D. Gaiotto and Y. Tachikawa, Liouville correlation functions from four-dimensional gauge theories, Lett. Math. Phys. 91 (2010) 167 [arXiv:0906.3219] [INSPIRE].

[6] H. Awata and H. Kanno, Instanton counting, Macdonald functions and the moduli space of D-branes, JHEP 05 (2005) 039 [hep-th/0502061] [INSPIRE].

[7] H. Awata and H. Kanno, Refined BPS state counting from Nekrasov's formula and Macdonald functions, Int. J. Mod. Phys. A 24 (2009) 2253 [arXiv:0805.0191] [InSPIRE].

[8] H. Awata et al., Anomaly in RTT relation for DIM algebra and network matrix models, Nucl. Phys. B 918 (2017) 358 [arXiv:1611.07304] [INSPIRE].

[9] H. Awata et al., Generalized Knizhnik-Zamolodchikov equation for Ding-Iohara-Miki algebra, Phys. Rev. D 96 (2017) 026021 [arXiv:1703.06084] [INSPIRE].

[10] H. Awata, H. Kanno, A. Mironov, A. Morozov, K. Suetake and Y. Zenkevich, $(q, t)-K Z$ equations for quantum toroidal algebra and Nekrasov partition functions on ALE spaces, JHEP 03 (2018) 192 [arXiv:1712.08016] [INSPIRE].

[11] H. Awata, H. Kanno, A. Mironov, A. Morozov, K. Suetake and Y. Zenkevich, The MacMahon R-matrix, JHEP 04 (2019) 097 [arXiv:1810. 07676] [INSPIRE].

[12] F. Aprile, S. Pasquetti and Y. Zenkevich, Flipping the head of $T[\mathrm{SU}(N)]$ : mirror symmetry, spectral duality and monopoles, JHEP 04 (2019) 138 [arXiv:1812.08142] [INSPIRE]. 
[13] M. Aganagic and S. Shakirov, Gauge/vortex duality and AGT, in New dualities of supersymmetric gauge theories, J. Teschner ed., Springer, Cham, Switzerland (2016), pg. 419 [arXiv: 1412.7132] [INSPIRE].

[14] H. Awata and Y. Yamada, Five-dimensional AGT conjecture and the deformed Virasoro algebra, JHEP 01 (2010) 125 [arXiv:0910.4431] [INSPIRE].

[15] M. Bershtein, B. Feigin and G. Merzon, Plane partitions with a "pit": generating functions and representation theory, Selecta Math. 24 (2018) 21 [arXiv:1512.08779].

[16] M. Bullimore, H.-C. Kim and P. Koroteev, Defects and quantum Seiberg-Witten geometry, JHEP 05 (2015) 095 [arXiv: 1412.6081] [inSPIRE].

[17] I. Burban and O. Schiffmann, On the Hall algebra of an elliptic curve, I, Duke Math. J. 161 (2012) 1171 [math.AG/0505148].

[18] J.-T. Ding and K. Iohara, Generalization and deformation of Drinfeld quantum affine algebras, Lett. Math. Phys. 41 (1997) 181 [INSPIRE].

[19] P. Etingof, I. Frenkel and A. Kirillov, Lectures on representation theory and Knizhnik-Zamolodchikov equations, Math. Surv. Monogr. 58, American Mathematical Soc., U.S.A. (1998).

[20] B. Feigin, E. Feigin, M. Jimbo, T. Miwa and E. Mukhin, Quantum continuous $\mathfrak{g l}_{\infty}$ : semiinfinite construction of representations, Kyoto J. Math. 51 (2011) 337 [arXiv: 1002 .3100].

[21] B. Feigin, K. Hashizume, A. Hoshino, J. Shiraishi and S. Yanagida, A commutative algebra on degenerate $C P^{1}$ and Macdonald polynomials, J. Math. Phys. 50 (2009) 095215 [arXiv: 0904.2291].

[22] B. Feigin, M. Jimbo, T. Miwa and E. Mukhin, Quantum toroidal $\mathfrak{g l}_{1}$-algebra: plane partitions, Kyoto J. Math. 52 (2012) 621 [arXiv:1110.5310].

[23] B. Feigin, M. Jimbo, T. Miwa and E. Mukhin, Finite type modules and Bethe ansatz for quantum toroidal $\mathfrak{g l}_{1}$, Commun. Math. Phys. 356 (2017) 285 [arXiv: 1603.02765] [INSPIRE].

[24] M. Fukuda, Y. Ohkubo and J. Shiraishi, Generalized Macdonald functions on Fock tensor spaces and duality formula for changing preferred direction, Commun. Math. Phys. $\mathbf{3 8 0}$ (2020) 1 [arXiv: 1903.05905] [INSPIRE].

[25] M. Fukuda, Y. Ohkubo and J. Shiraishi, Non-stationary Ruijsenaars functions for $\kappa=t^{-1 / N}$ and intertwining operators of Ding-Iohara-Miki algebra, SIGMA 16 (2020) 116 [arXiv: 2002.00243] [INSPIRE].

[26] I.B. Frenkel and N.Y. Reshetikhin, Quantum affine algebras and holonomic difference equations, Commun. Math. Phys. 146 (1992) 1 [INSPIRE].

[27] M. Ghoneim, C. Kozçaz, K. Kurşun and Y. Zenkevich, 4d higgsed network calculus and elliptic DIM algebra, arXiv:2012.15352 [INSPIRE].

[28] A. Iqbal, C. Kozcaz and C. Vafa, The refined topological vertex, JHEP 10 (2009) 069 [hep-th/0701156] [INSPIRE].

[29] A. Iqbal, C. Kozcaz and S.-T. Yau, Elliptic Virasoro conformal blocks, arXiv:1511.00458 [INSPIRE].

[30] M. Jimbo and T. Miwa, Algebraic analysis of solvable lattice models, CBMS Regional Conf. Ser. Math. 85, American Mathematical Soc., U.S.A. (1994).

[31] K. Miki, $A(q, \gamma)$ analog of the $W_{1+\infty}$ algebra, J. Math. Phys. 48 (2007) 123520. 
[32] A. Mironov and A. Morozov, On AGT relation in the case of U(3), Nucl. Phys. B $\mathbf{8 2 5}$ (2010) 1 [arXiv:0908.2569] [INSPIRE].

[33] A. Morozov, An analogue of Schur functions for the plane partitions, Phys. Lett. B 785 (2018) 175 [arXiv: 1808.01059] [INSPIRE].

[34] H. Nakajima, Lectures on Hilbert schemes of points on surfaces, Univ. Lect. Ser. 18, American Mathematical Soc., U.S.A. (1999).

[35] N.A. Nekrasov, Seiberg-Witten prepotential from instanton counting, Adv. Theor. Math. Phys. 7 (2003) 831 [hep-th/0206161] [INSPIRE].

[36] A. Negut, The R-matrix of the quantum toroidal algebra, arXiv:2005.14182 [INSPIRE].

[37] F. Nieri, An elliptic Virasoro symmetry in 6d, Lett. Math. Phys. 107 (2017) 2147 [arXiv: 1511.00574] [INSPIRE].

[38] A. Nedelin, S. Pasquetti and Y. Zenkevich, $T[\mathrm{SU}(N)]$ duality webs: mirror symmetry, spectral duality and gauge/CFT correspondences, JHEP 02 (2019) 176 [arXiv:1712.08140] [INSPIRE].

[39] H. Nakajima and K. Yoshioka, Instanton counting on blowup. I. 4-dimensional pure gauge theory, Invent. Math. 162 (2005) 313 [math. AG/0306198] [INSPIRE].

[40] Y. Saito, Elliptic Ding-Iohara algebra and the free field realization of the elliptic Macdonald operator, arXiv:1301.4912.

[41] F.A. Smirnov, Form factors in completely integrable models of quantum field theory, World Scientific, Singapore (1992).

[42] M. Taki, Surface operator, bubbling Calabi-Yau and AGT relation, JHEP 07 (2011) 047 [arXiv: 1007.2524] [INSPIRE].

[43] N. Wyllard, $A_{N-1}$ conformal Toda field theory correlation functions from conformal $N=2$ $\mathrm{SU}(N)$ quiver gauge theories, JHEP 11 (2009) 002 [arXiv:0907.2189] [INSPIRE].

[44] Y. Yoshida and K. Sugiyama, Localization of three-dimensional $N=2$ supersymmetric theories on $S^{1} \times D^{2}$, PTEP 2020 (2020) 113B02 [arXiv:1409.6713] [INSPIRE].

[45] Y. Zenkevich, 3d field theory, plane partitions and triple Macdonald polynomials, JHEP 06 (2019) 012 [arXiv: 1712.10300] [INSPIRE].

[46] Y. Zenkevich, Higgsed network calculus, arXiv:1812.11961 [INSPIRE].

[47] Y. Zenkevich, Mixed network calculus, arXiv:2012.15563 [INSPIRE].

[48] R. Flume and R. Poghossian, An algorithm for the microscopic evaluation of the coefficients of the Seiberg-Witten prepotential, Int. J. Mod. Phys. A 18 (2003) 2541 [hep-th/0208176] [INSPIRE].

[49] P. Longhi, F. Nieri and A. Pittelli, Localization of $4 d N=1$ theories on $\mathbb{D}^{2} \times \mathbb{T}^{2}$, JHEP 12 (2019) 147 [arXiv: 1906. 02051] [INSPIRE].

[50] A. Pittelli, Supersymmetric localization of refined chiral multiplets on topologically twisted $H^{2} \times S^{1}$, Phys. Lett. B 801 (2020) 135154 [arXiv:1812.11151] [INSPIRE].

[51] D. Maulik and A. Okounkov, Quantum groups and quantum cohomology, Astérisque 408, Société Mathématique de France, France (2019) [arXiv:1211.1287] [INSPIRE].

[52] A. Okounkov and A. Smirnov, Quantum difference equation for Nakajima varieties, arXiv: 1602.09007 [INSPIRE]. 